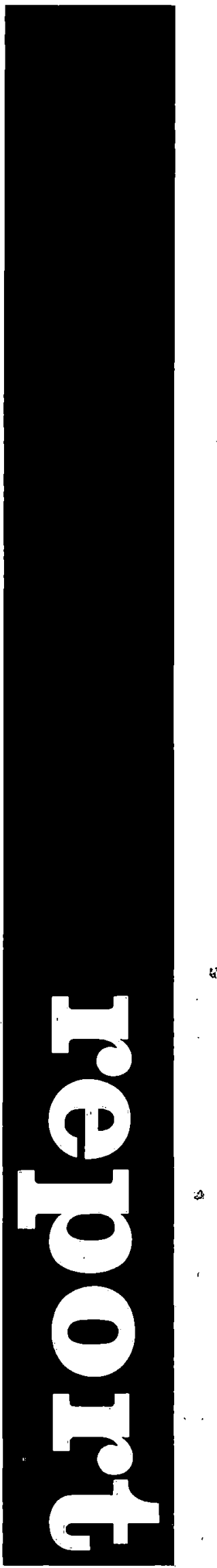

\title{
Engineering
}

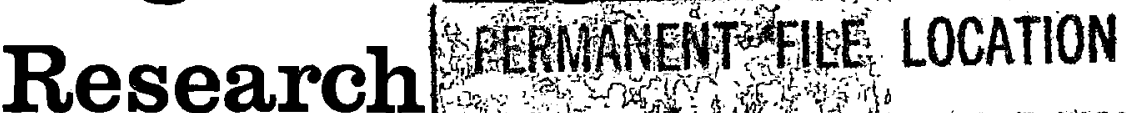
Institute
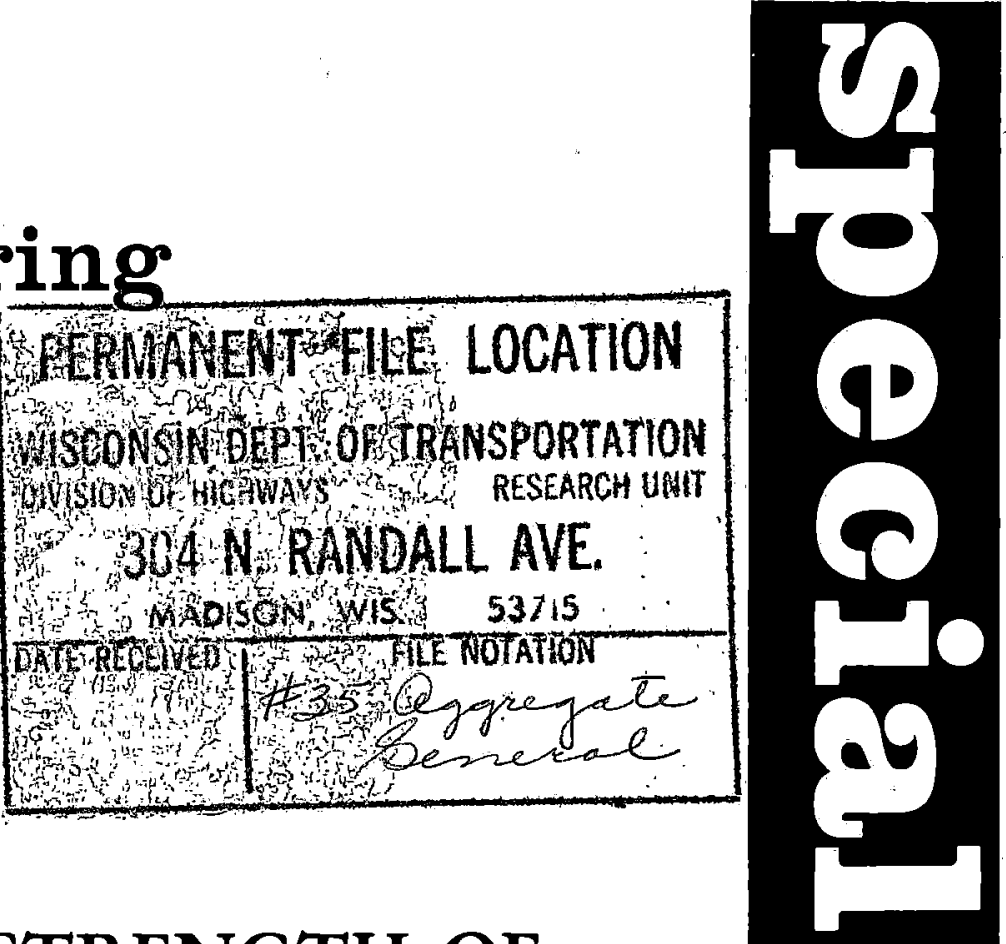

\section{SHEAR STRENGTH OF}

\section{GRANULAR MATERIALS}

F. H. Tinoco, Graduate Assistant

and

R. L. Handy, Professor

Department of Civil Engineering November 1967

Iowa State University 
ENGINEERING RESEARCH INSTITUTE

Iowa State University

\title{
SHEAR STRENGTH OF \\ GRANULAR MATERIALS
}

\author{
F. H. Tinoco, Graduate Assistant \\ and \\ R. L. Handy, Professor \\ Department of Civil Engineering
}

Contribution No. 67-9 of the

Soil Research Laboratory Engineering Research Institute lowa State University

The opinions, findings and conclusions expressed in this publication are those of the authors and not necessarily those of the lowa State Highway Commission nor the Bureau of Public Roads, U.S. Department of Commerce. 


\section{CONTENTS}

INTRODUCTION

Page

REVIEW OF L ITERATURE

PART I. SHEAR STRENGTH OF GRANULAR MATERIALS

THEORETICAL INVESTIGATION

10

Analys is of Particle Movements during Shear

10

Requirements for Sliding at Group Contacts

16

Mechanical Work

19

Application to Plane Strain, Triaxial Compression and Extension Test

Determination of the Angle of Solid Friction

Interpretation of the Parameter $\Omega$

TESTING OF THE THEORY

SUMMARY

1 PART II. SHEAR STRENGTH OF GRUSHED LIMESTONES 69

MATERIALS

METHODS OF INVESTIGATION

Triaxial Specimen Preparation

Triaxial Apparatus

Test Errors

DISCUSSION OF TEST RESULTS

CONCLUS IONS

ACKNOWLEDGEMENTS

RE FERENCES 


\section{INTRODUCTION}

Variable and somewhat unpredictable service records of Iowa crushed limestones used as base courses for flexible pavements indicated the need for a study of factors affecting the shear strength and deformationa1 behavior of these materials.

Crushed limestones may be considered within the general class of granular materials. Granular materials are particle assembilies which are devoid of interparticle cohesion, and where the individual particles are independent of each other except for frictional interaction and

Definition Granuar Material geometric constraints incidental to the packing of the assemblies.

The purpose of this investigation was to evaluate the effect of the frictional interaction between the particles and the effect of the geometric constraints among these particles on the shear strength of granular materials. The first step was to develop a theory to allow a separate consideration of the two mechanisms. The second step was to test the theory against available published data on granular materials; and the third step was to study the shear strength and deformational behavior of the Iowa crushed limestones in the 1ight of the proposed theory. 


\section{REVIEW OF LITERATURE}

Man has recognized the existence of friction for a long time. The first known written remarks on the nature of the laws that govern the phenomenon were by Leonardo da Vinci (1452-1519). Leonardo da Vinci proposed that friction was directly proportional to the normal force between sliding surfaces and that it was independent of the contact area between the surfaces, as reported by MacCurdy (1938).

These 1aws were rediscovered by Amontons (1699). However, Amontons ${ }^{1}$ Laws did not gain acceptance until they were confirmed and again proposed by Coulomb (1781). Coulomb was the first to distinguish between static and kinetic friction, and he established the independence of the coefficient of friction from the velocity of sliding.

Terzaghi (1925) proposed that the frictional force developed between two unlubricated surfaces was the result of molecular bonds formed at the contacts between the surfaces. Terzaghi made two assumptions; namely, that the real contact area is directly proportional to the normal load and that the shear strength at the contacts is independent of the normal 1oad. Thus, Terzaghi's theory of friction is expressed by the following two equations:

$$
\begin{aligned}
& F=A^{\prime} S^{\prime} \\
& \mu=S^{\prime} / P
\end{aligned}
$$

where $F$ is the frictional resistance, $A^{\prime}$ is the real contact area for inelastic behavior, $\mathrm{S}^{\prime}$ is the shear strength per unit area of the molecular bond, $\mu$ is the coefficient of friction, and $p$ is the pressure per unit of real contact area. 
The laws of friction have been further clarified in recent years by Bowden and Tabor (1950). They found that the real contact area between two bodies pressed together was much smaller than the apparent area of contact and that, in fact, adhesion takes place between adjacent (roughness)

surfaces at contacts between asperities. Under any level of the applied loads, these asperities yield plastically, so that the normal stress at a real contact is a constant equal to the yield stress of the material. Thus, the real contact area becomes directly proportional to the applied load, confirming Terzaghi's first assumption. The tangential force required to shear the junctions at the real contact is then proportional to the area of real contact. Thus,

$$
\begin{aligned}
& A^{\prime}=N / P_{m}^{\prime}, \\
& F=A^{\prime} S^{\prime}, \\
& F=N \cdot S^{\prime} / P m=N \cdot \mu, \text { and } \\
& \mu=S^{\prime} / P_{m}
\end{aligned}
$$

where $\mathrm{P}_{\mathrm{m}}$ is the yield pressure at the real contact.

Therefore, according to Bowden and Tabor the coefficient of friction depends on the nature or composition of the sliding surfaces in contact.

The oldest and still most widely used expression for soil shear strength is the Coulomb failure criterion,

$$
s=c+\sigma_{f} \tan \phi
$$

where $c$ is the cohesion, $\sigma_{f}$ the normal stress on the failure surface, and $\phi$ the angle of internal friction. 
The combination of Coulomb failure criterion with Mohr's theory of mechanical strength, 1 ater modified by Terzaghi (1923) in terms of the effective principal stresses, is given by:

$$
\sigma_{1}^{\prime}=\sigma_{3}^{\prime} \tan ^{2}(45+\phi / 2)+2 c \tan (45+\phi / 2)
$$

where $\sigma_{1}{ }^{\prime}$ and $\sigma_{3}{ }^{\prime}$. are the major and minor effective principal stresses respective1y. In soil mechanics "effective" stress designates tota1 stress less pore pressure; for example, $\sigma^{\prime}=\sigma-u$.

The value of $\phi$ or $\tan \phi$ as determined by the Mohr-Coulomb theory . is dependent on mode of packing of the assembly, experimental technique, stress history, angularity of grains, initial void ratio, and the level of the applied confining pressures. Therefore, even if tan $\phi$ is a function of the coefficient of solid friction between the particles, the determination of the latter is not possible from the former, and $\tan \phi$ is merely a parameter dependent on the conditions of the assembly during the experiment.

Mohr-Coulomb theory is strictly applicable to a body which shears without changing its volume. Reynolds (1885) showed that dense sands expand at failure, a phenomenon which he named dilatancy, whereas loose sands contract during shear to failure. Reynolds' experiments demonstrated that particle movements during deformation are not necessarily in the direction of the applied shear stresses, and indicated the effect of geometric constraints on the shear strength of granular materials.

Taylor (1948) was the first to attempt the separation of the strength component due to friction from that due to expansion, using 
data from shear box tests on sands. Skempton and Bishop (1950) also attempted this separation. The procedure in each case was to calculate the work done in expanding the sample by an amount $\delta \mathrm{v}$ per unit area against a vertical pressure $\sigma_{n}$, and equate this work to an equivalent shear component $T_{D}$ acting horizontally through a distance $\delta \Delta$, equal to the relative displacement of the two halves of the box. The difference between the maximum applied shear $\tau$ and $\tau_{D}$ was expressed in terms of a residual angle $\phi_{r}$ :

$$
\tan \phi_{r}=\frac{T-T_{D}}{\sigma_{n}}=\tan \phi_{\max }-\frac{\delta v}{\delta \Delta} .
$$

An expression based on the same principle was later presented by Bishop (1954), for use with the triaxial compression test, in the form:

$$
\tan ^{2}\left(45+\frac{1}{2} \phi_{r}\right)=\left(\frac{\sigma_{1}^{\prime}}{\sigma_{3}{ }^{\prime}}\right)_{\max }-\sigma_{3}^{\prime}\left(\frac{\delta \mathrm{v}}{\delta \varepsilon_{1}}\right) \text { rate of maiger principal }
$$

where $\delta v$ is the rate of unit volume change and $\delta \varepsilon_{1}$ is the rate of major principal strain change.

Newland and Allely (1957) considered the resultant direction of movement occurring during dilatation and determined a value of $\phi$ which they denoted $\phi_{f}$, given by:

$$
\phi_{\max }=\varphi_{f}+\theta
$$

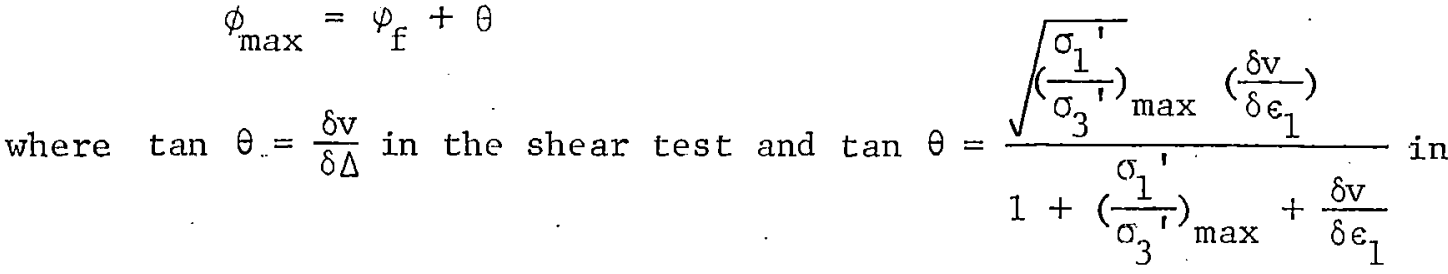

the triaxial test.

The derivation of $\phi_{\max }=\phi_{\mathrm{f}}+\theta$ was based on the assumption that 
the value of $\theta$ is a constant throughout the surface of sliding when the maximum shear stress has been reached, where $\theta$ represents the angle of inclination of the sliding surface with the direction of the shear force in the case of the direct shear test.

The values of $\phi_{f}$ and $\phi_{r}$ differed considerably, even though both values were derived to measure the same physical quantity (Newland and Allely, 1957).

Rowe (1962) discussed the behavior of ideal packings of spherical particles subjected to a major effective principal stress $\sigma_{1}^{\prime}$ and equal minor effective principal stresses $\sigma_{2}{ }^{\prime}=\sigma_{3}{ }^{\prime}$. He derived a stress-dilatancy relation for these packings given by:

$$
\sigma_{1}^{\prime} / \sigma_{3}^{\prime}=\tan \alpha \tan \left(\phi_{\mu}+\beta\right)
$$

where $\alpha$ is the packing characteristic of the ideal assembly and $\phi_{\mu}$ is the true angle of friction.

Rowe also derived an energy ratio given by

$$
\dot{\mathrm{E}}=\frac{\sigma_{1}^{\prime} \dot{\varepsilon}_{1}}{2 \sigma_{3}^{\prime} \dot{\varepsilon}_{3}}=\frac{\sigma_{1}^{\prime}}{\sigma_{3}^{\prime}\left(1+\mathrm{d} \dot{\mathrm{v}} / \mathrm{v} \dot{\varepsilon}_{1}\right)}=\frac{\tan \left(\phi_{\mu}+\beta\right)}{\tan \beta}
$$

where for comparison with previously presented expressions $\mathrm{d} \dot{\mathrm{v}} / \mathrm{v} \dot{\varepsilon}_{1}=$ $\delta v^{*} / \delta \epsilon_{1}$

Rowe observed that $\alpha$, the packing characteristic of the ideal assembly, had disappeared in his energy ration equation. Thus, he proceeded

* The expression $d \dot{v} / v \dot{\epsilon}_{1}$ is not identical to $\delta v / \delta \epsilon_{1}$. Rowe, Barden, and Lee (1964) changed this expression to $\delta \mathrm{v} / \delta \epsilon_{1}$. 
Energy Supplied (Loading)

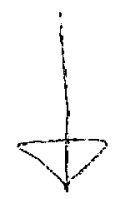

Energy Absorbed in Friction (Neat) Energy Absorbed in Rearranging (Strain) 
to derive the critical angle of sliding between particles in a random assembly of particles by postulating that the ratio of energy absorbed in internal friction to energy supplied, namely, $\dot{E}$, was a minimum. The value of the critical angle of sliding obtained by this procedure is equal to $45-1 / 2 \phi_{\mu}$, which substituted in the equation of the energy ratio, $\dot{E}$, led to

$$
\dot{\mathrm{E}}=\frac{\sigma_{1}{ }^{\prime} \dot{\varepsilon}_{1}}{2 \sigma_{3}{ }^{\prime} \dot{\varepsilon}_{3}}=\frac{\sigma_{1}^{\prime}}{\sigma_{3}{ }^{\prime}\left(1+\mathrm{d} \dot{\mathrm{v}} / \mathrm{v} \dot{\epsilon}_{1}\right)}=\tan ^{2}\left(45+\frac{1}{2} \phi_{\mu}\right)
$$

where $\quad \frac{\dot{\varepsilon}_{1}}{2 \dot{\varepsilon}_{3}}=\frac{1}{1+d \dot{v} / v \dot{\varepsilon}_{1}}$.

Rowe's experiments conducted on randomly packed masses of steel, glass, or quartz particles in which the physical properties were measured independently, showed that the minimum energy ratio criterion is closely obeyed by highly dilatant, dense, over-consolidated and reloaded assemblies throughout deformation to failure. However, the value of $\phi$ to satisfy the theory increases to $\phi_{f}$ when loose packings are considered because of additional energy losses due to rearranging of loose particles. Rowe found that $\phi_{\mathrm{u}} \leq \phi_{\mathrm{f}} \leq \phi_{\mathrm{cV}}$ where $\phi_{\mathrm{cV}}$ is the calculated value of $\phi$ when the sample reached the stage of zero rate of volume change. The angle $\phi_{\mathrm{cv}}$ was found to differ from $\phi_{\mathrm{u}}$ by 5 to 7 degrees in the case of sands.

Rowe (1963) applied the stress-dilatancy theory to the stability of earth masses behind retaining walls, in slopes and in foundations. Gibson and Morgenstern (1963), Trollope and Parkin (1963), Roscoe and Schofield (1964), and Scott (1964) discussed the stress-dilatancy theory postulated by Rowe (1962) and their criticism was mainly directed 
toward: (1) the assumed mechanism of deformation; (2) the assumed absence of rolling; (3) the assumption that the energy ratio $\dot{E}$ is a minimum in a random assembly of particles; and (4) the meaning of the " $\alpha$ planes" in a random assembly of particles.

Rowe, Barden, and Lee (1964) applied the stress-dilatancy relation to the case of the triaxial extension test and the direct shear test. The stress-dilatancy relation for use with the triaxial extension test was found to be:

$$
\frac{\sigma_{1}^{\prime}\left(1+d \dot{v} / v \dot{\varepsilon}_{1}\right)}{\sigma_{3}^{\prime}}=\tan ^{2}\left(45+\frac{\phi f}{2}\right)
$$

and for the direct shear test

$$
\begin{aligned}
& \phi_{\mathrm{E}}+\theta=\phi \text { and } \\
& \tan \theta=\frac{\delta \mathrm{v}}{\delta \Delta}
\end{aligned}
$$

The latter expression is identical to that derived by Newland and Allely (1957) for use with the direct shear test.

Rowe's theory has been substantiated by Horne (1965) who did not restrict his analysis to an idealized packing. Horne analyzed a randomly packed particulate assembly, with assumptions summarized as follows: (1) The particles are rotund and rigid with a constant coefficient of solid friction. (2) Deformation occurs as a relative motion between groups of particles but rolling motion is not admitted between the groups of particles. Horne obtained the expression for the energy ratio $\dot{\mathrm{E}}$ by writing a virtual work equation for the input $\sigma_{1}{ }^{\prime} \dot{\epsilon}_{1}$. Then, he minimized this ratio to obtain the value of $\beta_{c}=45-1 / 2 \phi_{u}$ which then led to 


$$
\dot{E}=\frac{\sigma_{1}^{\prime} \dot{\varepsilon}_{1}}{\sigma_{2}{ }^{\prime} \dot{\varepsilon}_{2}+\sigma_{3}{ }^{\prime} \dot{\varepsilon}_{3}}=\tan ^{2}\left(45+\frac{1}{2} \phi_{\mu}\right) \text {. }
$$

For the triaxial compression test with $\sigma_{2}^{\prime}=\sigma_{3}^{\prime}$ and $\dot{\epsilon}_{2}=\dot{\epsilon}_{3}$ this reduces to Rowe's equation. Horne thus established the limitations of the stress-dilatancy theory and concluded that the equation of the energy ratio $\dot{\mathrm{E}}$ that provided a relationship between the work quantities $\sigma_{1}^{\prime} \dot{\epsilon}, \sigma_{2}{ }^{\prime} \dot{\epsilon}_{2}$, and $\sigma_{3}{ }^{\prime} \dot{\epsilon}_{3}$ does not provide a relationship between stress. or strain rates separately. He also concluded that the relation may not apply to a highly compact assembly with a high degree of interlocking. 


\section{PART I. SHEAR STRENGTH OF GRANULAR MATERIALS}

\section{THEORETICAL INVESTIGATION}

The coefficient of solid friction between two particles is defined as $\mu=\tan \phi_{\mathrm{S}}=\mathrm{F} / \mathrm{N}$ where $\mathrm{F}$ denotes the frictional force, $\mathrm{N}$ is the force normal to the surface of sliding, and $\phi_{S}$ is the angle of solid friction. The coefficient of solid friction is considered independent of the normal force applied to the surfaces in contact and independent of the sliding

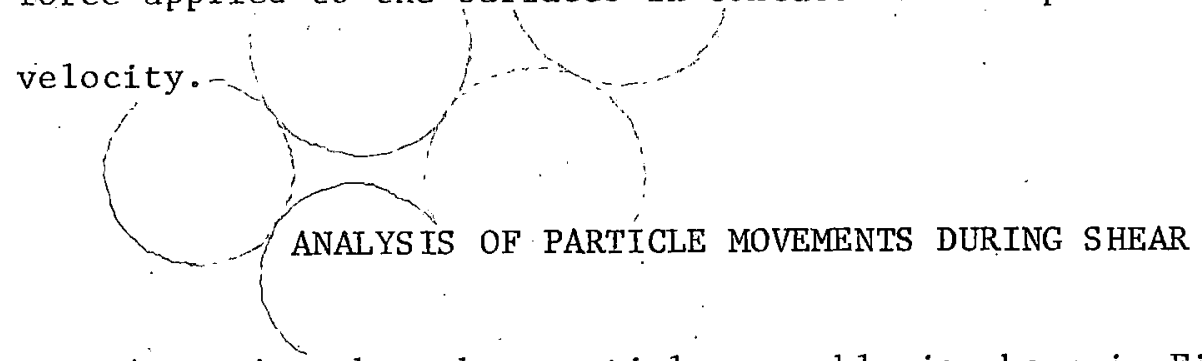

A section through a particle assembly is shown in Fig. 1a. The particles are drawn spherical for simplicity, but the analysis that follows is independent of the shape of the particles provided that their surfaces are predominantly convex.

The particle assembly is subjected to a force $\mathrm{N}$, applied in the vertical direction and a force $\mathrm{S}$, applied in the horizontal direction. Force $S$ causes particles $1,2,3$, etc., to move to the left relative to particles $1^{\prime}, 2^{\prime}, 3^{\prime}$, etc. If grain failure is excluded, then for particle 1 to move relative to particle $1^{\prime}$, it must initially slide along the direction of the tangent at the point of contact of the two particles; For example, in a direction making an angle $\beta_{1}$ to the direction of the horizontal force. Similar arguments may be made for the other particles, 2, 3, etc. 


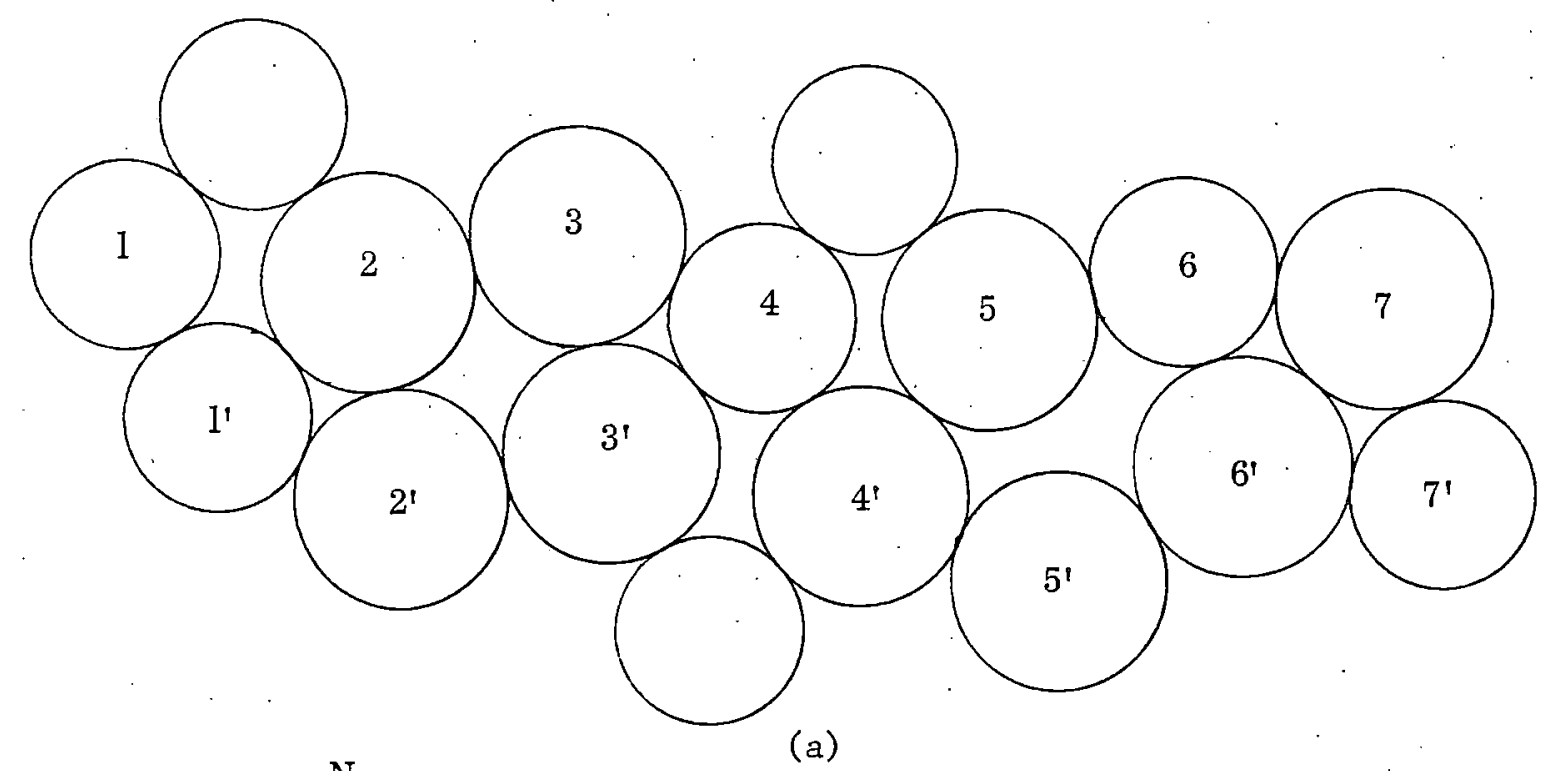

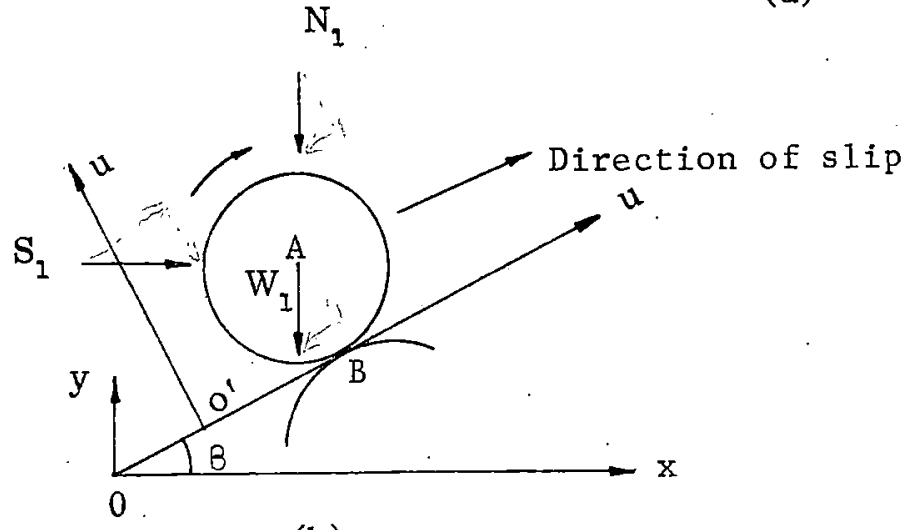

(b)

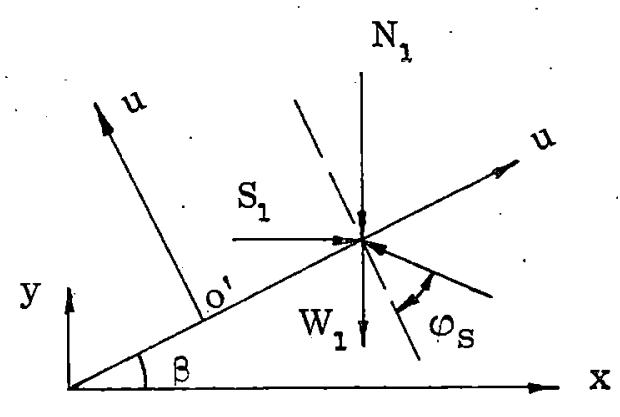

(c)

Fig. 1. Planar representation of a particle assembly and a free-body diagram for one particle. 


\section{Sliding}

Consider the single surface of sliding corresponding to particles 1 and $1^{\prime}$, Fig. Ib; resolving forces parallel and perpendicular to this surface:

$$
\begin{aligned}
& \sum F_{O^{\prime} \mathrm{v}}:\left(W_{1}+\mathrm{N}_{1}\right) \cos \beta_{1}+\mathrm{S}_{1} \sin \beta_{1}=\mathrm{R}_{1} \cos \phi_{\mathrm{s}} \\
& \sum \mathrm{F}_{\mathrm{O}^{\prime} \mathrm{u}}: \mathrm{S}_{1} \cos \beta_{1}-\left(\mathrm{W}_{1}+\mathrm{N}_{1}\right) \sin \beta_{1}=\mathrm{R}_{1} \sin \phi_{\mathrm{s}} .
\end{aligned}
$$

Eliminating $\mathrm{R}_{1}$ from Eqs. (la) and (1b):

$$
\begin{aligned}
& s_{1} \cos \beta_{1}-\left(w_{1}+N_{1}\right) \sin \beta_{1}=\left[\left(w_{1}+N_{1}\right) \cos \beta_{1}\right. \\
& \left.+s_{1} \sin \beta_{1}\right] \tan \phi_{s}
\end{aligned}
$$

and

$$
\tan \phi_{s}=\frac{s_{1}-\left(w_{1}+N_{1}\right) \tan \beta_{1}}{s_{1} \tan \beta_{1}+\left(N_{1}+w_{1}\right)}
$$

where $\phi_{S}$ is the angle of solid friction and $\tan \phi_{S}=\mu=$ coefficient of solid friction.

Equation (2) may be transformed to:

$$
\mathrm{S}_{1}=\left(\mathrm{W}_{1}+\mathrm{N}_{1}\right) \tan \left(\phi_{\mathrm{S}}+\beta_{1}\right)
$$

Similar solutions are found for particles 2 , 3 , etc.

If sliding occurs in the opposite direction, Eq. (2a) becomes:

$$
S_{1}=\left(W_{1}+N_{1}\right) \tan \left(\beta-\phi_{S}\right)
$$




\section{Rolling}

Consider particle 1 rolling over particle $1^{\prime}$ along the plane making an angle $\beta_{1}$ with the horizontal plane.

Figures $1 b$ and $1 c$ show the directions of translation and rotation of particle 1 and the free-body diagram.

Then,

$$
\begin{aligned}
& \sum F_{O^{\prime} \mathrm{u}}: \frac{W_{1}}{\mathrm{~g}} \ddot{\mathrm{u}}_{\mathrm{A}}=\mathrm{S}_{1} \cos \beta_{1}-\left(\mathrm{N}_{1}+\mathrm{W}_{1}\right) \sin \beta_{1}-\mathrm{R}_{1} \sin \phi \\
& \Sigma F_{\mathrm{O}^{\prime} \mathrm{v}}: \frac{\mathrm{W}_{1}}{\mathrm{~g}} \ddot{\mathrm{v}}_{\mathrm{A}}=-\mathrm{S}_{1} \sin \beta_{1}-\left(\mathrm{N}_{1}+\mathrm{W}_{1}\right) \cos \beta_{1}+\mathrm{R}_{1} \cos \phi \\
& \Sigma \mathrm{M}_{\mathrm{A}}: \frac{\mathrm{W}_{1}}{\mathrm{~g}} \mathrm{i}_{\mathrm{A}}^{2} \ddot{\theta}=\mathrm{R}_{1} \mathrm{r} \sin \phi
\end{aligned}
$$

where $r$ is the radius of particle $1, i_{A}^{2}$ is the radius of gyration of the particle 1 with respect to its geometric axis, and $\phi$ is a corresponding friction angle given by $\phi<\phi_{S}$. That is, the acting frictional force is less than: the frictional force required for sliding to take place.

The condition that there is no sliding requires that the relative velocity of the point of contact at any instant is zero. That is, point $B$ is the instantaneous center of rotation. From this it follows that the angular velocity of rotation of the particle is $\dot{\theta}=\dot{u}_{A} / r$, from which, by differentiation, $\ddot{\theta}=\ddot{u}_{A} / r$. Substituting this in Eq. (3c):

$$
\frac{W_{1}}{g} i_{A}^{2} \frac{\ddot{\mathrm{u}}_{\mathrm{A}}}{\mathrm{r}}=\mathrm{R}_{1} \mathrm{r} \sin \phi \text {. }
$$

Eliminating the friction force $R_{1}$ sin $\phi$ between Eqs. (3d) and (3a): 


$$
\ddot{u}_{A}=\frac{1}{1+i_{A}^{2} / r^{2}} \frac{g}{w_{1}}\left[s_{1} \cos \beta_{1}-\left(N_{1}+w_{1}\right) \sin \beta_{1}\right] \text {. }
$$

Then,

$$
R_{1} \sin \phi=\frac{i_{A}^{2} / r^{2}}{1+i_{A}^{2} / r^{2}}\left[S_{1} \cos \beta_{1}-\left(N_{1}+W_{1}\right) \sin \beta_{1}\right]
$$

and 1 et

$$
\frac{i_{A}^{2} / r^{2}}{1+i_{A}^{2} / r^{2}}=C
$$

and

$$
R_{1} \sin \phi=C\left[s_{1} \cos \beta_{1}-\left(N_{1}+W_{1}\right) \sin \cdot \beta_{1}\right] \text {. }
$$

From Eq. (3b), the value of $R_{1}$ is equal to

$$
\frac{s_{1} \sin \beta_{1}+\left(N_{1}+W_{1}\right) \cos \beta_{1}}{\cos \phi_{S}} \text {. }
$$

Substituting the value of $R_{1}$ in $\mathrm{Eq}$. (3f)

$$
\left[s_{1} \tan \beta_{1}+\left(N_{1}+W_{1}\right)\right] \tan \phi=C\left[s_{1}-\left(N_{1}+w_{1}\right) \tan \beta_{1}\right]
$$

which on rearrangement gives:

$$
\frac{\mathrm{S}_{1}}{\mathrm{~N}_{1}+\mathrm{W}_{1}}=\frac{\tan \phi+\mathrm{C} \tan \beta_{1}}{\mathrm{C}-\tan \beta_{1} \tan \phi}=\frac{1 / \mathrm{c} \tan \phi+\tan \beta_{1}}{1-1 / \mathrm{c} \tan \beta_{1} \tan \phi}
$$

and let $1 / \mathrm{C} \tan \phi=\tan \phi_{\mathrm{r}}$, then $\mathrm{Eq}$. (3i) is transformed to give:

$$
\frac{\mathrm{S}_{1}}{\mathrm{~N}_{1}+\mathrm{w}_{1}}=\tan \left(\phi_{\mathrm{r}}+\beta_{1}\right) \text {. }
$$

If rolling occurs in the opposite direction, then Eq. (3j) is given by:

$$
\frac{S_{1}}{N_{1}+W_{1}}=\tan \left(\beta_{1}-\phi_{r}\right)
$$

If rotation occurs in a counter-clockwise direction, Eqs. (3j) and (3k) are given, respectively, by: 


$$
\begin{aligned}
& \frac{S_{1}}{N_{1}+W_{1}}=\tan \left(\beta_{1}-\phi r_{1}\right) \\
& \frac{S_{1}}{N_{1}+W_{1}}=\tan \left(\phi r_{1}+\beta_{1}\right)
\end{aligned}
$$

where $\tan \phi \mathrm{r}_{1}=1 / \mathrm{C}_{1}$ tan $\phi$ and $\mathrm{C}_{1}=\frac{\mathrm{i}_{\mathrm{A}}^{2} / \mathrm{r}^{2}}{1-\mathrm{i}_{\mathrm{A}}^{2} / \mathrm{r}^{2}}$.

\section{Sliding Versus Rolling}

The ratio $S_{1} / N_{1}+W_{1}$ is either a function of $\beta$ and $\phi_{s}$ when sliding is about to occur, or a function of $\beta$ and $\phi_{r}$ when rolling is about to occur, where $\phi_{r}$ is either equal to $\phi_{r}$ or to $\phi_{r I}$.

Consider a particle assembly containing $\mathrm{P}$ particles in a state of equilibrium under a vertical force $\mathrm{N}$ and a horizontal force $\mathrm{S}$. An increment of the horizontal force $S$ is applied producing motions within the assembly until equilibrium is reached. These motions will consist of relative motions between groups of particles; for example, these groups of particles will slide over each other for very small distances until sliding ceases. Then, relative motion between any two individual particles depends entirely on the relative motion between two adjacent groups containing the particles. This relative motion will consist of
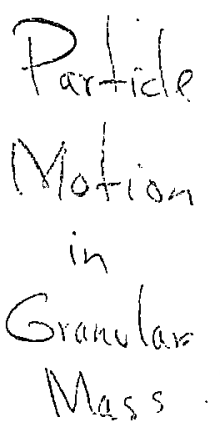
a combination of sliding and rolling, the result being that the total volume of voids will either increase or decrease. Vol of viois One may classify the contacts between particles or groups of will Olange particles as sliding contacts or nonsliding contacts. The process by which a particle assembly passes from one state of equilibrium to another consists of the disappearance of the initial groups of sliding contacts and the formation of new groups of potential sliding contacts. 
That is, the proportion of sliding and nonsliding contacts is modified when the particle assembly reaches a new state of equilibrium. The modification of the ratio of total number of sliding contacts to total number of nonsliding contacts results in either a decrease or an increase in the total volume of voids of the particle assembly.

\section{REQUIREMENTS FOR SLIDING AT GROUP CONTACTS}

Sliding at group contacts will occur in some preferential direction readily found from considerations of the stresses at a point referred to a principal stress coordinate-axis system.

Let

$$
\sigma_{1}^{\prime}>\sigma_{2}^{\prime}>\sigma_{3}^{\prime}
$$

Then,

$$
\begin{aligned}
& \tau_{n}^{2}+\left(\sigma_{n}^{\prime}-\frac{\sigma_{2}^{\prime}+\sigma_{3}^{\prime}}{2}\right)^{2} \geqq\left(\frac{\sigma_{2}^{\prime}-\sigma_{3}^{\prime}}{2}\right)^{2} \\
& \tau_{n}^{2}+\left(\sigma_{n}^{\prime}-\frac{\sigma_{1}^{\prime}+\sigma_{3}^{\prime}}{2}\right)^{2} \leqq\left(\frac{\sigma_{1}^{\prime}-\sigma_{3}^{\prime}}{2}\right)^{2} \\
& \tau_{n}^{2}+\left(\sigma_{n}^{\prime}-\frac{\sigma_{1}^{\prime}+\sigma_{2}^{\prime}}{2}\right)^{2} \geqq\left(\frac{\sigma_{1}^{\prime}-\sigma_{2}^{\prime}}{2}\right)^{2}
\end{aligned}
$$

It is apparent from Eqs. (4a), (4b), and (4c) that the absolute maximum shearing stress is $\tau_{n}=\sigma_{1}^{\prime}-\sigma_{3}^{\prime} / 2$ and it occurs at $\dot{\sigma}_{n}^{\prime}=$ $\sigma_{1}^{\prime}+\sigma_{3}^{\prime} / 2$. Thus, the sliding contacts in the granular assembly will be oriented in plane parallel to the $\sigma_{1}^{\prime}, \sigma_{3}^{\prime}$ plane.

Selecting the equality sign in Eq. (4b),

$$
\tau_{n}^{2}+\left(\sigma_{n}^{\prime}-\frac{\sigma_{1}^{\prime}+\sigma_{3}^{\prime}}{2}\right)^{2}=\left(\frac{\sigma_{1}^{\prime}-\sigma_{3}^{\prime}}{2}\right)^{2} .
$$


Equation (4d) is the equation of a circle which is referred to as a Mohr circle. This circle can also be given in parametric form introducing the parameter $2 \beta$ where $\beta$ represents the angle which the given plane makes with the major principal plane. Then

$$
\begin{aligned}
& \sigma^{\prime}=\frac{\sigma_{1}^{\prime}+\sigma_{3}^{\prime}}{2}+\left(\frac{\sigma_{1}^{\prime}-\sigma_{3}^{\prime}}{2}\right) \cos 2 \beta \\
& \tau_{n}=\left(\frac{\sigma_{1}^{\prime}-\sigma_{3}^{\prime}}{2}\right) \sin 2 \beta .
\end{aligned}
$$

Sliding contacts in a preferential direction were defined as making a critical angle $\beta_{c}$ with a given plane. Next evaluate this critical angle.

Sliding will take place when

$$
\tau_{n}=\sigma_{n}^{\prime} \tan \phi_{s}
$$

where all terms have been previously defined.

Substituting Eq. (4g) in Eq. (4f),

$$
\begin{aligned}
& \sigma_{n}^{\prime} \tan \phi_{s}=\left(\frac{\sigma_{1}^{\prime}-\sigma_{3}^{\prime}}{2}\right) \sin 2 \beta, \\
& \sigma_{n}^{\prime}=\left(\frac{\sigma_{1}^{\prime}-\sigma_{3}^{\prime}}{2}\right) \frac{\sin 2 \beta}{\tan \phi_{s}}
\end{aligned}
$$

and substituting Eq. (4h) in Eq. (4e),

$$
\left(\frac{\sigma_{1}^{\prime}-\sigma_{3}^{\prime}}{2}\right) \frac{\sin 2 \beta}{\tan \phi_{s}}=\frac{\sigma_{1}^{\prime}+\sigma_{3}^{\prime}}{2}+\left(\frac{\sigma_{1}^{\prime}-\sigma_{3}^{\prime}}{2}\right) \cos 2 \beta
$$

and on rearranging

$$
\frac{\sigma_{1}^{\prime}}{\sigma_{3}^{\prime}}=1+\frac{1}{\frac{1}{2} \frac{\sin 2 \beta}{\tan \phi_{s}}-\cos ^{2} \beta} \text {. }
$$


The critical value of $\beta$ will be a maximum for sliding to take place, as previously shown. A maximum $\beta$ value will make the ratio $\sigma_{1}{ }^{\prime} / \sigma_{3}{ }^{\prime}$ a minimum. Thus, maximizing the denominator of the second right-hand term of Eq. $(4 j)$ :

$$
\begin{aligned}
& \frac{\mathrm{d}}{\mathrm{d} \beta}\left(\frac{1}{2} \frac{\sin 2 \beta}{\tan \phi_{s}}-\cos ^{2} \beta\right)=0, \\
& \cos 2 \beta+\sin 2 \beta \tan \phi_{s}=0 \\
& \tan \left(-\phi_{S}\right)=\cot 2 \beta
\end{aligned}
$$

and

$$
2 \beta=90+\phi_{s}
$$

or

$$
\beta=45+\frac{\phi_{S}}{2}
$$

Substituting Eq. (4k) in Eq. (4j),

$$
\frac{\sigma_{1}^{\prime}}{\sigma_{3}^{\prime}}=\frac{1+\sin \phi_{s}}{1=\sin \phi_{s}}=\tan ^{2}\left(45+\frac{\phi_{s}}{2}\right) \text {. }
$$

Thus, for sliding to take place at group contacts, the value of the stress ratio $\sigma_{1}{ }^{\prime} / \sigma_{3}^{\prime}$ is given by Eq. (4m).

Equation (4m) is identical with the Mohr-Coulomb criteria. However, Mohr's theory requires that an envelope be drawn tangent to the Mohr circles representing the maximum stress ratio, and Coulomb theory requires that such an envelope is required. The purpose of the previous analysis is to determine whether a sphere in an inclined plane will roll or slide. Equation $(4 \mathrm{~m})$ gives the condition for sliding rather than rolling to take place on a given plane at an angle $\beta=45+1 / 2 \phi_{S}$. 
MECHANICAL WORK

When a body is deformed by a system of external forces in equilibrium, the mechanical work done by them is equal to the work consumed by the internal stresses.

In the analysis of the mechanical work done by the external forces and the work consumed by the internal stresses in a particle assembly, two assumptions are made:

1. The directions of principal stresses and principal strains coincide with each other at any and at every instant during deformation.

2. Energy absorbed in particle deformation is neglected. That is, any elastic and/or plastic deformation of the particle is neglected as a result of which the particle is assumed to behave as a rigid body.

The state of stress is given through the effective principal stresses denoted by $\sigma_{1}{ }^{\prime}, \sigma_{2}{ }^{\prime}, \sigma_{3}{ }^{\prime}$, and their directions and the change in the state of strain is defined by the principal strains $\delta \epsilon_{1}, \delta \epsilon_{2}$, $\delta \epsilon_{3}$, whose directions coincide instantaneously with the principal directions of stress. Compressive stresses and strains are considered negative.

If the mechanical work is denoted by $W$ per unit volume of material, the increment $\delta \mathrm{W}$ of the work done at a given instant by the principal stresses is equal to:

$$
\delta W=\sigma_{1}^{\prime} \delta \epsilon_{1}+\sigma_{2}^{\prime} \delta \epsilon_{2}-\sigma_{3}^{\prime} \delta \epsilon_{3}
$$

In confined compression testing of granular materials, it is common to subject the sample to an all-around pressure and apply loads in the directions of the principal stresses. A common procedure is to 
let the minor principal stress, $\sigma_{3}{ }^{\prime}$, remain equal to the initial allaround pressure.

Therefore, the principal stresses may be expressed by

$$
\begin{aligned}
& \sigma_{1}^{\prime}=\sigma_{3}^{\prime}+\left(\sigma_{1}^{\prime}-\sigma_{3}^{\prime}\right), \\
& \sigma_{2}^{\prime}=\sigma_{3}^{\prime}+\left(\sigma_{2}^{\prime}-\sigma_{3}^{\prime}\right), \\
& \sigma_{3}^{\prime}=\sigma_{3}^{\prime} .
\end{aligned}
$$

Thus, the granular material will reach equilibrium under an al1around pressure, $\sigma_{3}{ }^{\prime}$, and then, the sample is subjected to the stresses $\left(\sigma_{1}^{\prime}-\sigma_{3}^{\prime}\right)$ and $\left(\sigma_{2}^{\prime}-\sigma_{3}^{\prime}\right)$.

Then the increment of work $\delta W_{e}$ applied to the system is given by:

$$
\delta W_{e}=\left(\sigma_{1}^{\prime}-\sigma_{3}^{\prime}\right) \delta \varepsilon_{1}+\left(\sigma_{2}^{\prime}-\sigma_{3}^{\prime}\right) \delta \epsilon_{2}
$$

The increment of internal work absorbed by the system is equal to:

$$
\delta W_{i}=\sigma_{1}^{\prime} \delta \epsilon_{1}+\sigma_{2}^{\prime} \delta \epsilon_{2}-\sigma_{3}^{\prime} \delta \epsilon_{3}
$$

$$
\text { Granular materials are known to change in volume during a shear }
$$

process. Therefore, let $\mathrm{v}$ be the change in volume per unit volume, considered negative when the sample volume is decreased, and $\delta \mathrm{v}$ be an increment of the change in volume per unit volume. The increment of change in volume per unit volume is equal to:

$$
-\delta \mathrm{v}=-\delta \epsilon_{1}-\delta \epsilon_{2}+\delta \epsilon_{3} .
$$

Then, $\delta \epsilon_{3}=-\delta \mathrm{v}+\delta \epsilon_{1}+\delta \epsilon_{2}$ and the increment of internal work is given by: 


$$
\delta \mathrm{W}_{\mathrm{i}}=\left(\sigma_{1}^{\prime} \cdot-\sigma_{3}^{\prime}\right) \delta \epsilon_{1}+\left(\sigma_{2}^{\prime}-\sigma_{3}^{\prime}\right) \delta \epsilon_{2}+\sigma_{3}^{\prime} \delta \mathrm{v}
$$

The applied stresses produce both a change in volume and sliding due to friction within the granular assembly. Thus, the increment of internal work absorbed by the granular assembly may be separated into two components which will be referred to as frictional, $\delta W i_{f}$ and dilatancy, $\delta W i_{D}$.

Then,

$$
\delta W i=\delta W i_{E}+\delta W i_{D}
$$

and

$$
\delta W i_{f}+\delta W i_{D}=\left(\sigma_{1}^{\prime}-\sigma_{3}^{\prime}\right) \delta \epsilon_{1}+\left(\sigma_{2}^{\prime}-\sigma_{3}^{\prime}\right) \delta \epsilon_{2}+\sigma_{3}^{\prime} \delta \mathrm{v}
$$

Consider a granular assembly composed of frictionless particles. If a system of stresses is applied to this assembly, the increment of internal work absorbed by the assembly is equal to:

$$
\delta W i_{D}=\left(\sigma_{1}^{\prime}-\sigma_{3}^{\prime}\right) \delta \epsilon_{1 D}+\left(\sigma_{2}^{\prime}-\sigma_{3}^{\prime}\right) \delta \epsilon_{2 D}+\sigma_{3}^{\prime} \delta \dot{v}_{D}
$$

where $\delta \epsilon_{1 D}, \delta \epsilon_{2 D}$, and $\delta \epsilon_{3 D}$ are the increments of principal strains absorbed by the assembly as a result of which a volume change is registered within the assembly.

Similarly,

$$
\delta \mathrm{Wi}=\left(\sigma_{1}^{\prime}-\sigma_{3}^{\prime}\right) \delta \epsilon_{1 \mathrm{f}}+\left(\sigma_{2}^{\prime}-\sigma_{3}^{\prime}\right) \delta \epsilon_{2 \mathrm{f}}+\sigma_{3}{ }^{\prime} \delta \mathrm{v}_{\mathrm{f}} \cdot
$$

Substitutions. of Eq. (6b) and (6a) in the left-hand side of Eq. (6a) give 


$$
\begin{aligned}
\left(\sigma_{1}^{\prime}\right. & \left.-\sigma_{3}^{\prime}\right) \delta \epsilon_{1 f}+\left(\sigma_{2}^{\prime}-\sigma_{3}^{\prime}\right) \delta \epsilon_{2 f}+\sigma_{3}^{\prime} \delta v_{f}+\left(\sigma_{1}^{\prime}-\sigma_{3}^{\prime}\right) \delta \varepsilon_{1 D} \\
& +\left(\sigma_{2}^{\prime}-\sigma_{3}^{\prime}\right) \delta \epsilon_{2 D}+\sigma_{3}^{\prime} \delta v_{D}=\left(\sigma_{1}^{\prime}-\sigma_{3}^{\prime}\right) \delta \epsilon_{1} \\
& +\left(\sigma_{2}^{\prime}-\sigma_{3}^{\prime}\right) \delta \epsilon_{2}+\sigma_{3}^{\prime} \delta \mathrm{v}
\end{aligned}
$$

The following relations are obtained from Eq. (6d):

$$
\begin{aligned}
& \delta \epsilon_{1}=\delta \epsilon_{1 \mathrm{f}}+\delta \epsilon_{1 \mathrm{D}}, \\
& \delta \epsilon_{2}=\delta \epsilon_{2 \mathrm{f}}+\delta \epsilon_{2 \mathrm{D}}, \\
& \delta \mathrm{v}=\delta \mathrm{v}_{\mathrm{f}}+\delta \mathrm{v}_{\mathrm{D}}, \\
& \delta \dot{v}_{\mathrm{D}}=\delta \epsilon_{1 \mathrm{D}}+\delta \epsilon_{2 \mathrm{D}}+\delta \epsilon_{3 \mathrm{D}}, \\
& \delta \mathrm{v}_{\mathrm{f}}=\delta \epsilon_{1 \mathrm{f}}+\delta \epsilon_{2 \mathrm{f}}-\delta \epsilon_{3 \mathrm{f}}, \\
& \delta \epsilon_{3}=\delta \epsilon_{3 \mathrm{f}}-\delta \epsilon_{3 \mathrm{D}} .
\end{aligned}
$$

and

Sliding within a granular assembly may be considered analogous to the sliding between a block and a plane surface which are perfectly smooth, as a result of which the term $\delta v_{f}$ is equal to zero. Then,

$$
\delta W_{i f}=\left(\sigma_{1}^{\prime}-\sigma_{3}^{\prime}\right) \delta \varepsilon_{1 f}+\left(\sigma_{2}^{\prime}-\sigma_{3}^{\prime}\right) \delta \epsilon_{2 f}
$$

and

$$
\begin{aligned}
\delta w_{i} & =\left(\sigma_{1}^{\prime}-\sigma_{3}^{\prime}\right) \delta \epsilon_{1 \mathrm{f}}+\left(\sigma_{2}^{\prime}-\sigma_{3}^{\prime}\right) \delta \epsilon_{2 \mathrm{f}}+\left(\sigma_{1}^{\prime}-\sigma_{3}^{\prime}\right) \delta \epsilon_{1 \mathrm{D}} \\
& +\left(\sigma_{2}^{\prime}-\sigma_{3}^{\prime}\right) \delta \epsilon_{2 \mathrm{D}}+\sigma_{3}^{\prime} \delta \mathrm{v}_{\mathrm{D}} .
\end{aligned}
$$

Or

$$
\begin{aligned}
\delta W_{i}= & \left(\sigma_{1}^{\prime}-\sigma_{3}^{\prime}\right) \delta \epsilon_{1 f}+\left(\sigma_{2}^{\prime}-\sigma_{3}^{\prime}\right) \delta \epsilon_{2 f}+\left(\sigma_{1}^{\prime}-\sigma_{3}^{\prime}\right) \delta \epsilon_{1 D} \\
& +\left(\sigma_{2}^{\prime}-\sigma_{3}^{\prime}\right) \delta \epsilon_{2 D}+\sigma_{3}^{\prime} \delta \mathrm{v} .
\end{aligned}
$$


Since $\delta W_{e}=\delta W_{i}$,

$$
\begin{aligned}
& \left(\sigma_{1}^{\prime}-\sigma_{3}^{\prime}\right) \delta \varepsilon_{1}+\left(\sigma_{2}^{\prime}-\sigma_{3}^{\prime}\right) \delta \varepsilon_{2}=\left(\sigma_{1}^{\prime}-\sigma_{3}^{\prime}\right) \delta \varepsilon_{1 \mathrm{f}} \\
& \quad+\left(\sigma_{2}^{\prime}-\sigma_{3}^{\prime}\right) \delta \varepsilon_{2 \mathrm{f}}+\left(\sigma_{1}^{\prime}-\sigma_{3}^{\prime}\right) \delta \varepsilon_{1 \mathrm{D}}+\left(\sigma_{2}^{\prime}-\sigma_{3}^{\prime}\right) \delta \varepsilon_{2 \mathrm{D}} \\
& \quad+\sigma_{3}^{\prime} \delta \mathrm{v}
\end{aligned}
$$

and

$$
\begin{aligned}
\delta \mathrm{W}_{1 \mathrm{f}} & =\left(\sigma_{1}^{\prime}-\sigma_{3}^{\prime}\right) \delta \varepsilon_{1 \mathrm{f}}+\left(\sigma_{2}^{\prime}-\sigma_{3}^{\prime}\right) \delta \varepsilon_{2 \mathrm{f}}=\left(\sigma_{1}^{\prime}-\sigma_{3}^{\prime}\right) \delta \varepsilon_{1} \\
& +\left(\sigma_{2}^{\prime}-\sigma_{3}^{\prime}\right) \delta \varepsilon_{2}-\left(\sigma_{1}^{\prime}-\sigma_{3}^{\prime}\right) \delta \varepsilon_{1 \mathrm{D}}-\left(\sigma_{2}^{\prime}-\sigma_{3}^{\prime}\right) \delta \varepsilon_{2 \mathrm{D}} \\
& -\sigma_{3}^{\prime} \delta \mathrm{v} .
\end{aligned}
$$

Let

$$
\begin{aligned}
& \sigma_{1 f}^{\prime}=\sigma_{3}^{\prime}+\left(\sigma_{1}^{\prime}-\sigma_{3}^{\prime}\right)-\left(\sigma_{1}^{\prime}-\sigma_{3}^{\prime}\right)_{D}, \\
& \sigma_{2 f}^{\prime}=\sigma_{3}^{\prime}+\left(\sigma_{2}^{\prime}-\sigma_{3}^{\prime}\right)-\left(\sigma_{2}^{\prime}-\sigma_{3}^{\prime}\right)_{D}, \\
& \sigma_{3 f}^{\prime}=\sigma_{3}^{\prime}-\sigma_{3 D}^{\prime},
\end{aligned}
$$

and

$$
\delta W i_{f}=\left(\sigma_{1 f}^{\prime}-\sigma_{3 f}^{\prime}\right) \delta \epsilon_{1}+\left(\sigma_{2 f}^{\prime}-\sigma_{3 f}^{\prime}\right) \delta \epsilon_{2} .
$$

Substituting Eqs. (7a), (7b), (7c) in the right-hand side of Eq. (7d),

$$
\begin{aligned}
\delta W i_{f} & =\left(\sigma_{1}^{\prime}-\sigma_{3}^{\prime}\right) \delta \epsilon_{1}-\left(\sigma_{1}^{\prime}-\sigma_{3}{ }^{\prime}\right)_{D} \delta \epsilon_{1}+\left(\sigma_{2}{ }^{\prime}-\sigma_{3}{ }^{\prime}\right) \delta \epsilon_{2} \\
& -\left(\sigma_{2}^{\prime}-\sigma_{3}^{\prime}\right){ }_{D} \delta \epsilon_{2}+\sigma_{3 D}\left(\delta \epsilon_{1}+\delta \epsilon_{2}\right),
\end{aligned}
$$

and using Eq. (6h), 


$$
\begin{gathered}
-\left(\sigma_{1}^{\prime}-\sigma_{3}^{\prime}\right)_{D} \delta \varepsilon_{1}-\left(\sigma_{2}^{\prime}-\sigma_{3}^{\prime}\right)_{D} \delta \epsilon_{2}+\sigma_{3 D}^{\prime}\left(\delta \epsilon_{1}+\delta \epsilon_{2}\right) \\
=-\left(\sigma_{1}^{\prime}-\sigma_{3}^{\prime}\right) \delta \epsilon_{1 D}-\left(\sigma_{2}^{\prime}-\sigma_{3}^{\prime}\right) \delta \epsilon_{2 D}-\sigma_{3}^{\prime} \delta \mathrm{v} .
\end{gathered}
$$

Therefore,

$$
\begin{aligned}
& \left(\sigma_{1}^{\prime}-\sigma_{3}^{\prime}\right)_{\mathrm{D}}=\left(\sigma_{1}^{\prime}-\sigma_{3}^{\prime}\right) \frac{\delta \epsilon_{1 \mathrm{D}}}{\delta \epsilon_{1}}, \\
& \left(\sigma_{2}^{\prime}-\sigma_{3}^{\prime}\right)_{\mathrm{D}}=\left(\sigma_{2}^{\prime}-\sigma_{3}^{\prime}\right) \frac{\delta \epsilon_{2 \mathrm{D}}}{\delta \epsilon_{2}} \\
& \sigma_{3 \mathrm{D}}^{\prime}=-\sigma_{3}^{\prime} \frac{\delta \mathrm{v}}{\delta \epsilon_{1}+\delta \epsilon_{2}}
\end{aligned}
$$

Substituting the values obtained in Eqs. $(7 \mathrm{~g}),(7 \mathrm{~h})$, and (7i) in the corresponding Eqs. (7a), (7b), and (7c),

$$
\begin{aligned}
\sigma_{1 \mathrm{f}} & =\sigma_{3}^{\prime}+\left(\sigma_{1}^{\prime}-\sigma_{3}^{\prime}\right)-\left(\sigma_{1}^{\prime}-\sigma_{3}^{\prime}\right) \frac{\delta \epsilon_{1 D}}{\delta \epsilon_{1}} \\
& =\sigma_{1}^{\prime}-\left(\sigma_{1}^{\prime}-\sigma_{3}^{\prime}\right) \frac{\delta \epsilon_{1 D}}{\delta \epsilon_{1}} . \\
\sigma_{2 f}^{\prime} & =\sigma_{3}^{\prime}+\left(\sigma_{2}^{\prime}-\sigma_{3}^{\prime}\right)-\left(\sigma_{2}^{\prime}-\sigma_{3}^{\prime}\right) \frac{\delta \epsilon_{2 D}}{\delta \epsilon_{2}} \\
& =\sigma_{2}^{\prime}-\left(\sigma_{2}^{\prime}-\sigma_{3}^{\prime}\right) \frac{\delta \epsilon_{2 D}}{\delta \epsilon_{2}} . \\
\sigma_{3 f}^{\prime} & =\sigma_{3}^{\prime}+\sigma_{3}^{\prime} \frac{\delta v}{\delta \epsilon_{1}+\delta \epsilon_{2}}=\sigma_{3}^{\prime}\left(1+\frac{\delta v}{\delta \epsilon_{1}+\delta \epsilon_{2}}\right) .
\end{aligned}
$$

Equations $(7 j),(7 k)$, and $(7 \ell)$ provide the values of the principal. stresses corresponding to friction.

APPLICATION TO PLANE STRAIN, TRIAXIAL COMPRESSION AND TRIAXIAL EXTENS ION TESTS

Consider a granular assembly subjected to plane strain conditions. 
The evaluation of $\sigma_{1 f^{\prime}}^{\prime}, \sigma_{2}^{\prime}$, and $\sigma_{3 f}^{\prime}$ is performed in a similar manner as described in the previous section.

The following conditions apply during a plane strain test:

$$
\sigma_{1}^{\prime}>\sigma_{2}^{\prime}>\sigma_{3}^{\prime}
$$

and

$$
\epsilon_{2}=0
$$

Therefore, $\delta \epsilon_{2}=0=\delta \epsilon_{2 \mathrm{f}}+\delta \epsilon_{2 \mathrm{D}}$

and

$$
\delta \epsilon_{2 f}=-\delta \epsilon_{2 D} \cdot
$$

Then, Eq. (7f) is transformed to

$$
\begin{gathered}
-\left(\sigma_{1}^{\prime}-\sigma_{3}^{\prime}\right)_{\mathrm{D}} \delta \epsilon_{1}+\sigma_{3 \mathrm{D}}^{\prime} \delta \epsilon_{1}=-\left(\sigma_{1}^{\prime}-\sigma_{3}^{\prime}\right) \delta \epsilon_{1 \mathrm{D}} \\
-\left(\sigma_{2}^{\prime}-\sigma_{3}^{\prime}\right) \delta \epsilon_{2 \mathrm{D}}-\sigma_{3}^{\prime} \delta \mathrm{v} .
\end{gathered}
$$

To solve Eq. (8), it is required that a further assumption be made. Let $\delta \epsilon_{2 D}=\delta \epsilon_{3 D}$. That is, the state of strain during a pure dilation is symmetrical with respect to the intermediate and the minor principal strain.

Then, one may proceed as follows:

$$
\begin{aligned}
& \delta \mathrm{v}=\delta \epsilon_{1 \mathrm{D}}+2 \delta \epsilon_{3 \mathrm{D}} \\
& \delta \epsilon_{2 \mathrm{D}}=\delta \epsilon_{3 \mathrm{D}}=\frac{\delta \mathrm{v}-\delta \epsilon_{1 \mathrm{D}}}{2} \\
& -\left(\sigma_{1}^{\prime}-\sigma_{3}^{\prime}\right)_{\mathrm{D}} \delta \epsilon_{1}+\sigma_{3 \mathrm{D}^{\prime} \delta \epsilon_{1}}=-\left(\sigma_{1}^{\prime}-\sigma_{3}^{\prime}\right) \delta \epsilon_{1 \mathrm{D}} \\
& \quad-\left(\sigma_{2}^{\prime}-\sigma_{3}^{\prime}\right) \frac{\delta \mathrm{v}-\delta \epsilon_{1 \mathrm{D}}}{2}-\sigma_{3}^{\prime} \delta \mathrm{v},
\end{aligned}
$$

and 


$$
\begin{aligned}
-\left(\sigma_{1}^{\prime}-\sigma_{3}^{\prime}\right)_{D} \delta \epsilon_{1}+\sigma_{3 D} \delta \epsilon_{1}=-\left[\sigma_{1}^{\prime}-\frac{1}{2}\left(\sigma_{2}^{\prime}-\sigma_{3}^{\prime}\right)\right] \delta \epsilon_{1 D} \\
-\frac{1}{2}\left(\sigma_{2}^{\prime}+\sigma_{3}^{\prime}\right) \delta \mathrm{v} .
\end{aligned}
$$

Then,

$$
\begin{aligned}
& \left(\sigma_{1}^{\prime}-\sigma_{3}^{\prime}\right)_{\mathrm{D}}=\left[\sigma_{1}^{\prime}-\frac{1}{2}\left(\sigma_{2}^{\prime}+\sigma_{3}^{\prime}\right)\right] \frac{\delta \epsilon_{1 D}}{\delta \varepsilon_{1}} \\
& \sigma_{3 \mathrm{D}}^{\prime}=-\frac{1}{2}\left(\sigma_{2}^{\prime}+\sigma_{3}^{\prime}\right) \frac{\delta \mathrm{v}}{\delta \epsilon_{1}},
\end{aligned}
$$

and

$$
\begin{aligned}
& \sigma_{1 \bar{f}}^{\prime}=\sigma_{1}^{\prime}-\left[\sigma_{1}^{\prime}-\frac{1}{2}\left(\sigma_{2}^{\prime}+\sigma_{3}^{\prime}\right)\right] \frac{\delta \epsilon_{1 D}}{\delta \epsilon_{1}} \\
& \sigma_{3 f^{\prime}}=\sigma_{3}^{\prime}+\frac{1}{2}\left(\sigma_{2}^{\prime}+\sigma_{3}^{\prime}\right) \frac{\delta \mathrm{v}}{\delta \epsilon_{1}} .
\end{aligned}
$$

The corresponding Eqs. (8d) and (8e) for triaxial compression and triaxial extension tests are readily derived from these equations.

$$
\begin{aligned}
& \text { Triaxia1 compression test: }) \\
& \sigma_{1 f^{\prime}}=\sigma_{1}^{\prime}-\left(\sigma_{1}^{\prime}-\sigma_{3}^{\prime}\right) \frac{\delta \epsilon_{1 D}}{\delta \epsilon_{1}} \\
& \sigma_{3 E}^{\prime}=\sigma_{3}^{\prime}\left(1+\frac{\delta \mathrm{v}}{\delta \epsilon_{1}}\right)
\end{aligned}
$$

and in the case of triaxial extension test:

$$
\begin{aligned}
& \sigma_{3 f^{\prime}}=\sigma_{3}^{\prime}+\left(\sigma_{1}^{\prime}-\sigma_{3}^{\prime}\right) \frac{\delta \varepsilon_{3 D}}{\delta \varepsilon_{3}} \\
& \sigma_{1 f^{\prime}}=\sigma_{1}^{\prime}\left(1-\frac{\delta \mathrm{v}}{\delta \epsilon_{3}}\right) .
\end{aligned}
$$

Equations (8d) through (8i) will be used to determine the angle of sliding friction for these three types of test. 


\section{DETERMINATION OF THE ANGLE OF SOLID FRICTION}

The condition for sliding to take place between group contacts is:

$$
\frac{\sigma_{1 f}^{\prime}}{\sigma_{3 f}^{\prime}}=\frac{1+\sin \phi_{s}}{1-\sin \phi_{s}}
$$

and

$$
\sin \phi_{s}=\frac{\sigma_{1 f}^{\prime}-\sigma_{3 f}^{\prime}}{\sigma_{1 f}^{\prime}+\sigma_{3 f}^{\prime}}
$$

where the subscript 'f' stands for friction.

The values of $\sigma_{1 f}^{\prime}$ and $\sigma_{3 f}^{\prime}$ are given by Eqs. (8d) through (8i). Once a test is selected to evaluate the angle of solid friction of a particle assembly, the corresponding equation from (8d) through (8i) is selected and substituted in Eq. (9b).

Thus, for plane strain test,

$$
\sin \phi_{\mathrm{s}}=\frac{\left(\sigma_{1}^{\prime}-\sigma_{3}^{\prime}\right)-\left[\sigma_{1}^{\prime}-\frac{1}{2}\left(\sigma_{2}^{\prime}+\sigma_{3}^{\prime}\right)\right] \frac{\delta \epsilon_{1 \mathrm{D}}}{\delta \epsilon_{1}}-\frac{1}{2}\left(\sigma_{2}{ }^{\prime}+\sigma_{3}^{\prime}\right) \frac{\delta \mathrm{v}}{\delta \varepsilon_{1}}}{\left(\sigma_{1}^{\prime}+\sigma_{3}^{\prime}\right)-\left[\sigma_{1}^{\prime}-\frac{1}{2}\left(\sigma_{2}^{\prime}+\sigma_{3}^{\prime}\right) \frac{\delta \epsilon_{1 \mathrm{D}}}{\delta \epsilon_{1}}+\frac{1}{2}\left(\sigma_{2}^{\prime}+\sigma_{3}^{\prime}\right) \frac{\delta \mathrm{v}}{\delta \varepsilon_{1}}\right.}
$$

for triaxial compression test

$$
\sin \phi_{\mathrm{s}}=\frac{\left(\sigma_{1}^{\prime}-\sigma_{3}^{\prime}\right)-\left(\sigma_{1}^{\prime}-\sigma_{3}^{\prime}\right) \frac{\delta \epsilon_{1 D}}{\delta \epsilon_{1}}-\sigma_{3}^{\prime} \frac{\delta \mathrm{v}}{\delta \epsilon_{1}}}{\left(\sigma_{1}^{\prime}+\sigma_{3}^{\prime}\right)+\left(\sigma_{1}^{\prime}-\sigma_{3}^{\prime}\right) \frac{\delta \varepsilon_{1 D}}{\delta \epsilon_{1}}+\sigma_{3}^{\prime} \frac{\delta \mathrm{v}}{\delta \epsilon_{1}}}
$$

and triaxial extension test,

$$
\sin \phi_{s}=\frac{\left(\sigma_{1}^{\prime}-\sigma_{3}^{\prime}\right)-\left(\sigma_{1}^{\prime}-\sigma_{3}^{\prime}\right) \frac{\delta \epsilon_{3 D}}{\delta \epsilon_{3}}-\sigma_{1}^{\prime} \frac{\delta \mathrm{v}}{\delta \varepsilon_{1}}}{\left(\sigma_{1}^{\prime}+\sigma_{3}^{\prime}\right)+\left(\sigma_{1}^{\prime}-\sigma_{3}^{\prime}\right) \frac{\delta \epsilon_{3 D}}{\delta \epsilon_{3}}-\sigma_{1}^{\prime} \frac{\delta \mathrm{v}}{\delta \epsilon_{3}}}
$$

Equations (9c), (9d), and (9e) are rearranged to obtain respectively: 


$$
\begin{aligned}
& \frac{\sigma_{1}^{\prime}}{\sigma_{3}^{\prime}}--\frac{1}{2}\left(1+\frac{\sigma_{2}^{\prime}}{\sigma_{3}^{\prime}}\right) \frac{\delta \mathrm{v}}{\delta \epsilon_{1}}=\left[\frac{\sigma_{1}^{\prime}}{\sigma_{3}^{\prime}}-\frac{1}{2}\left(1+\frac{\sigma_{2}^{\prime}}{\sigma_{3}^{\prime}}\right)\right]\left(1-\sin \phi_{\mathrm{s}}\right) \frac{\delta \varepsilon_{1 \mathrm{D}}}{\delta \varepsilon_{1}} \\
&+\left[\frac{\sigma_{1}^{\prime}}{\sigma_{3}^{\prime}}+1+\frac{1}{2}\left(1+\frac{\sigma_{2}^{\prime}}{\sigma_{3}^{\prime}}\right) \frac{\delta \mathrm{v}}{\delta \epsilon_{1}}\right] \sin \phi_{\mathrm{s}}, \\
& \frac{\sigma_{1}^{\prime}}{\sigma_{3}^{\prime}}-\left(1+\frac{\delta \mathrm{v}}{\delta \epsilon_{1}}\right)=\left(\frac{\sigma_{1}^{\prime}}{\sigma_{3}^{\prime}}-1\right)\left(1-\sin \phi_{\mathrm{s}}\right) \frac{\delta \epsilon_{1 \mathrm{D}}}{\delta \epsilon_{1}} \\
&+\left[\frac{\sigma_{1}^{\prime}}{\sigma_{3}^{\prime}}+\left(1+\frac{\delta \mathrm{v}}{\delta \epsilon_{1}}\right)\right] \sin \phi_{\mathrm{s}}
\end{aligned}
$$

and

$$
\frac{\sigma_{1}^{\prime}}{\sigma_{3}^{\prime}}-\frac{1}{1-\frac{\delta \mathrm{v}}{\delta \epsilon_{3}}}=\left(\frac{\sigma_{1}^{\prime}}{\delta_{3}^{\prime}}-1\right)\left(\frac{1+\sin \phi_{\mathrm{s}}}{1-\frac{\delta \mathrm{v}}{\delta \epsilon_{3}}}\right) \frac{\delta \epsilon_{3 D}}{\delta \epsilon_{3}}+\left[\frac{\sigma_{1}^{\prime}}{\sigma_{3}^{\prime}}+\frac{1}{1-\frac{\delta \mathrm{v}}{\delta \epsilon_{3}}}\right] \sin \phi_{\mathrm{s}}
$$

Let

$$
\begin{aligned}
& \Omega_{\mathrm{P}}=\left[\frac{\sigma_{1}^{\prime}}{\sigma_{3}^{\prime}}-\frac{1}{2}\left(1+\frac{\sigma_{2}^{\prime}}{\sigma_{3}^{\prime}}\right)\right]\left(1-\sin \phi_{\mathrm{S}}\right) \frac{\delta \epsilon_{1 \mathrm{D}}}{\delta \epsilon_{1}}, \\
& \left.\Omega_{\mathrm{TC}}=\left[\frac{\sigma_{1}^{\prime}}{\sigma_{3}^{\prime}}-1\right)\left(1-\sin \phi_{\mathrm{S}}\right)\right] \frac{\delta \epsilon_{1 \mathrm{D}}}{\delta \epsilon_{1}}, \\
& \Omega_{\mathrm{TE}}^{\prime}=\left(\frac{\sigma_{1}^{\prime}}{\sigma_{3}^{\prime}}-1\right)\left(\frac{1+\sin \phi_{\mathrm{S}}}{1-\frac{\delta \mathrm{V}}{\delta \epsilon_{3}}}\right) \frac{\delta \epsilon_{3 \mathrm{D}}}{\delta \epsilon_{3}},
\end{aligned}
$$

and

$$
\Omega_{\mathrm{TE}}^{\prime}=\Omega_{\mathrm{TE}}\left(1-\frac{\delta \mathrm{v}}{\delta \epsilon_{3}}\right)=\left(\frac{\sigma_{1}^{\prime}}{\sigma_{3}^{\prime}}-1\right)\left(1+\sin \phi_{\mathrm{s}}\right) \frac{\delta \epsilon_{3 \mathrm{D}}}{\delta \epsilon_{3}}
$$

where the subscripts ' $\mathrm{P}$ ', 'TC', and 'TE' stand respectively for plane strain, triaxial compression and triaxial extension test.

Equations (9f), $(9 \mathrm{~g})$, and $(9 \mathrm{~h})$ are now expressed by:

$$
\frac{\sigma_{1}^{\prime}}{\sigma_{3}{ }^{\prime}}-1-\frac{1}{2}\left(1+\frac{\sigma_{2}^{\prime}}{\sigma_{3}{ }^{\prime}}\right) \frac{\delta \mathrm{v}}{\delta \varepsilon_{1}}=\Omega_{\mathrm{p}}+\left[\frac{\sigma_{1}^{\prime}}{\sigma_{3}{ }^{\prime}}+1+\frac{1}{2}\left(1+\frac{\sigma_{2}^{\prime}}{\sigma_{3}{ }^{\prime}}\right) \frac{\delta \mathrm{v}}{\delta \varepsilon_{1}}\right] \sin \phi_{\mathrm{s}},
$$




$$
\frac{\sigma_{1}^{\prime}}{\sigma_{3}^{\prime}}-\left(1+\frac{\delta \mathrm{v}}{\delta \varepsilon_{1}}\right)=\Omega_{\mathrm{TC}}+\left[\frac{\sigma_{1}^{\prime}}{\sigma_{3}^{\prime}}+\left(1+\frac{\delta \mathrm{v}}{\delta \epsilon_{1}}\right)\right] \sin \phi_{\mathrm{s}},
$$

and

$$
\frac{\sigma_{1}^{\prime}}{\sigma_{3}^{\prime}}-\frac{1}{1-\frac{\delta \mathrm{v}}{\delta \epsilon_{3}}}=\Omega_{\mathrm{TE}}+\left[\frac{\sigma_{1}^{\prime}}{\sigma_{3}^{\prime}}-\frac{1}{1-\frac{\delta \mathrm{v}}{\delta \epsilon_{3}}}\right] \sin \phi_{\mathrm{s}}
$$

There are two unknowns, $\phi_{\mathrm{s}}$ and $\Omega$, in each of the Eqs. (9m), (9n), and (9p). The determination of these two unknowns is possible by plotting the experimental data in the form as given by each of the respective equations. For example, in the case of the triaxial compression test, a plot of $\sigma_{1}{ }^{\prime} / \sigma_{3}{ }^{\prime}-\left(1+\delta \mathrm{v} / \delta \epsilon_{1}\right)$ versus $\sigma_{1}{ }^{\prime} / \sigma_{3}{ }^{\prime}+\left(1+\delta \mathrm{v} / \delta \epsilon_{1}\right)$ allowed the determination of $\phi_{S}$ and $\Omega_{\mathrm{TC}}$ if such a plot corresponds to a straight line of the form:

$$
\frac{\sigma_{1}^{\prime}}{\sigma_{3}^{\prime}}-\left(1+\frac{\delta \mathrm{v}}{\delta \epsilon_{1}}\right)=\Omega_{\mathrm{TC}}+\left[\frac{\sigma_{1}^{\prime}}{\sigma_{3}^{\prime}}+\left(1+\frac{\delta \mathrm{v}}{\delta \epsilon_{1}}\right)\right] \tan \psi
$$

where $\tan \psi=\sin \phi_{S}$, and $\Omega_{T C}=\Omega_{T C}$, over a wide range of the deformation process of the sample. The value of $\Omega_{\mathrm{TC}}$ is not in general a constant throughout the deformation process, but it may reach a value of $\Omega_{T C}$ which is constant over a given deformation range. If $\Omega_{\mathrm{TC}}$ reaches a constant value $\Omega_{\text {TC }}$ over a given range of the deformation process, it is then possible to determine the value of $\sin \phi_{S}$ and calculate the value of $\sin \phi_{S}$ corresponding to any given instant during the process of deformation of the sample.

INTERPRETATION OF THE PARAMETER $\Omega$

Regrouping terms in Eq. (9f): 


$$
\begin{aligned}
\frac{\sigma_{1}^{\prime}}{\sigma_{3}^{\prime}} & =\tan ^{2}\left(45+\frac{1}{2} \phi_{s}\right)+\frac{1}{2}\left(1+\frac{\sigma_{2}^{\prime}}{\sigma_{3}^{\prime}}\right) \frac{\delta \mathrm{v}}{\delta \epsilon_{1}} \tan ^{2}\left(45+\frac{1}{2} \phi_{s}\right) \\
& +\left[\frac{\sigma_{1}^{\prime}}{\sigma_{3}^{\prime}}-\frac{1}{2}\left(1+\frac{\sigma_{2}^{\prime}}{\sigma_{3}^{\prime}}\right] \frac{\delta \varepsilon_{1 D}}{\delta \varepsilon_{1}}\right.
\end{aligned}
$$

or

$$
\begin{aligned}
\frac{\sigma_{1}^{\prime}}{\sigma_{3}^{\prime}} & =\tan ^{2}\left(45+\frac{1}{2} \phi_{s}\right)+\frac{1}{2} \frac{\delta \mathrm{v}}{\delta \epsilon_{1}} \tan ^{2}\left(45+\frac{1}{2} \phi_{s}\right)+\left(\frac{\sigma_{1}^{\prime}}{\sigma_{3}^{\prime}}-\frac{1}{2}\right) \frac{\delta \epsilon_{1 D}}{\delta \epsilon_{1}} \\
& +\frac{1}{2} \frac{\sigma_{2}^{\prime}}{\sigma_{3}^{\prime}}\left[\frac{\delta \mathrm{v}}{\delta \epsilon_{1}} \tan ^{2}\left(45+\frac{1}{2} \phi_{s}\right)-\frac{\delta \epsilon_{1 D}}{\delta \epsilon_{1}}\right]
\end{aligned}
$$

Recalling that,

$$
\Omega_{\mathrm{P}}=\left[\frac{\sigma_{1}^{\prime}}{\sigma_{3}^{\prime}}-\frac{1}{2}\right] \frac{\delta \epsilon_{1 D}}{\delta \epsilon_{1}}\left(1-\sin \phi_{\mathrm{S}}\right)-\frac{1}{2} \frac{\sigma_{2}^{\prime}}{\sigma_{3}^{\prime}}\left(1-\sin \phi_{\mathrm{s}}\right) \frac{\delta \epsilon_{1 D}}{\delta \epsilon_{1}}
$$

then, let

$$
\begin{aligned}
& \Omega_{\mathrm{PD}}=\left[\frac{\sigma_{1}^{\prime}}{\sigma_{3}{ }^{\prime}}-\frac{1}{2}\right] \frac{\delta \epsilon_{1 \mathrm{D}}}{\delta \epsilon_{1}}-\frac{1}{2} \frac{\sigma_{2}^{\prime}}{\sigma_{3}^{\prime}} \frac{\delta \epsilon_{1 \mathrm{D}}}{\delta \epsilon_{1}}, \\
& \Omega_{\mathrm{PfD}}=\frac{1}{2} \frac{\sigma_{2}^{\prime}}{\sigma_{3}^{\prime}} \frac{\delta \epsilon_{1 \mathrm{D}}}{\delta \epsilon_{1}} \sin \phi_{\mathrm{S}}-\left[\frac{\sigma_{1}^{\prime}}{\sigma_{3}^{\prime}}-\frac{1}{2}\right] \frac{\delta \epsilon_{1 \mathrm{D}}}{\delta \epsilon_{1}} \sin \phi_{\mathrm{S}} ;
\end{aligned}
$$

where the subscript $D$ and $f D$ stand for dilatancy and friction due to dilatancy, respectively, and Eq. (10a) is now expressed by:

$$
\frac{\sigma_{1}^{\prime}}{\sigma_{3}^{\prime}}=\tan ^{2}\left(45+\frac{1}{2} \phi_{s}\right)+\frac{1}{2}\left(1+\frac{\sigma_{2}{ }^{\prime}}{\sigma_{3}{ }^{\prime}}\right) \frac{\delta \mathrm{v}}{\delta \epsilon_{1}} \tan ^{2}\left(45+\frac{\phi_{\mathrm{s}}}{2}\right)+\Omega_{\mathrm{PD}}{ }^{\prime}
$$

or

$$
\begin{aligned}
\frac{\sigma_{1}^{\prime}}{\sigma_{3}^{\prime}} & =\tan ^{2}\left(45+\frac{1}{2} \phi_{s}\right)+\frac{1}{2}\left(1+\frac{\sigma_{2}^{\prime}}{\sigma_{3}{ }^{\prime}}\right) \frac{\delta \mathrm{v}}{\delta \varepsilon_{1}} \tan ^{2}\left(45+\frac{1}{2} \phi_{\mathrm{S}}\right) \\
& +\Omega_{\mathrm{P}}+\Omega_{\mathrm{PfD}} .
\end{aligned}
$$

The corresponding equations in the case of triaxial compression test are given by: 


$$
\frac{\sigma_{1}^{\prime}}{\sigma_{3}{ }^{\prime}}=\tan ^{2}\left(45+\frac{1}{2} \phi_{S}\right)+\frac{\delta \mathrm{v}}{\delta \varepsilon_{1}} \tan ^{2}\left(45+\frac{1}{2} \phi_{S}\right)+\Omega_{T C D}
$$

and

$$
\frac{\sigma_{1}^{\prime}}{\sigma_{3}{ }^{\prime}}=\tan ^{2}\left(45+\frac{1}{2} \phi_{S}\right)+\frac{\delta v}{\delta \epsilon_{1}} \tan ^{2}\left(45+\frac{1}{2} \phi_{S}\right)+\Omega_{T C}+\Omega_{T C f D}
$$

where

$$
\Omega_{\mathrm{TCD}}=\left[\frac{\sigma_{1}^{\prime}}{\sigma_{3}^{\prime}}-1\right] \frac{\delta \epsilon_{1 D}}{\delta \epsilon_{1}}
$$

and

$$
\Omega_{\mathrm{TCfD}}=\left[\frac{\sigma_{1}^{\prime}}{\sigma_{3}^{\prime}}-1\right] \frac{\delta \varepsilon_{1 \mathrm{D}}}{\delta \epsilon_{1}} \sin \phi_{\mathrm{s}}
$$

The corresponding equations in the case of the triaxial extension test are given by:

$$
\frac{\sigma_{1}^{\prime}}{\sigma_{3}^{\prime}}=\frac{1}{1-\delta \mathrm{v} / \delta \epsilon_{3}}\left(1+\Omega_{\mathrm{TED}}\right) \tan ^{2}\left(45+\frac{1}{2} \phi_{\mathrm{s}}\right),
$$

and

$$
\frac{\sigma_{1}^{\prime}}{\sigma_{3}{ }^{\prime}}=\frac{1}{1-\delta \mathrm{v} / \delta \varepsilon_{3}}\left(1+\Omega_{\mathrm{TE}}-\Omega_{\mathrm{TE} f \mathrm{D}}\right) \tan ^{2}\left(45+\frac{1}{2} \phi_{\mathrm{S}}\right)
$$

where

$$
\dot{\Omega}_{\mathrm{TED}}=\left(\frac{\sigma_{1}^{\prime}}{\sigma_{3}^{\prime}}-1\right) \frac{\delta \epsilon_{3 \mathrm{D}}}{\delta \epsilon_{3}}
$$

and

$$
\Omega_{\mathrm{TEfD}}=\left(\frac{\sigma_{1}^{\prime}}{\sigma_{3}^{\prime}}-1\right) \frac{\delta \varepsilon_{3 D}}{\delta \epsilon_{3}} \sin \phi_{\mathrm{s}} \cdot
$$

The different form of Eq. (10l) with respect to Eqs. (10f) and $(10 \mathrm{~g})$ is due to the use of $\delta \epsilon_{3}$ rather than $\delta \epsilon_{1}$ for the derivation of 
the corresponding equations; the reason being that the minor natural principal strain, $\epsilon_{3}$, correspond to the axial strain measured, in a triaxial extension test.

Figure 2 is a model idealization of the terms encountered in Eq. $(10 \mathrm{~g})$

Figure 2 a represents two rigid blocks, whose coefficient of solid friction is $\mu=\tan \phi_{S}$, sliding against each other along a plane inclined $45+1 / 2 \phi_{\mathrm{s}}$ degrees with respect to the horizontal plane. The value of the ratio $\sigma_{1}{ }^{\prime} / \sigma_{3}{ }^{\prime}$ is then given by:

$$
\frac{\sigma_{1}^{\prime}}{\sigma_{3}^{\prime}}=\tan ^{2}\left(45+\frac{1}{2} \phi_{s}\right)
$$

which was obtained from

$$
\frac{\sigma_{1}^{\prime} b}{\sigma_{3}^{\prime} b \tan \left(45+\frac{\phi_{s}}{2}\right.}=\tan \left[\phi_{s}+\left(45-\frac{\phi_{s}}{2}\right)\right] \text {. }
$$

Consider that each block is mounted on a set of cylindrical rollers possessing the same frictional characteristic of the two blocks as shown in Fig. 2b. Relative movements of the two blocks occur along the contacts between the rollers and the value of $\sigma_{1}{ }^{\prime} / \sigma_{3}{ }^{\prime}$ is then given by:

$$
\frac{\sigma_{1}^{\prime}}{\sigma_{3}{ }^{\prime}}=\tan ^{2}\left(45+\frac{\phi_{s}}{2}\right)+\frac{\delta v}{\delta \epsilon_{1}} \tan ^{2}\left(45+\frac{1}{2} \phi_{s}\right)
$$

Consider, instead of two rigid blocks, an assembly of frictionless particles as shown in Fig. 2c. The required ratio $\sigma_{1}^{\prime} / \sigma_{3}^{\prime}$ to produce relative movements between the particles at a given instant is given by:

$$
\frac{\sigma_{1}^{\prime}}{\sigma_{3}^{\prime}}=\Omega_{\mathrm{TCD}}
$$



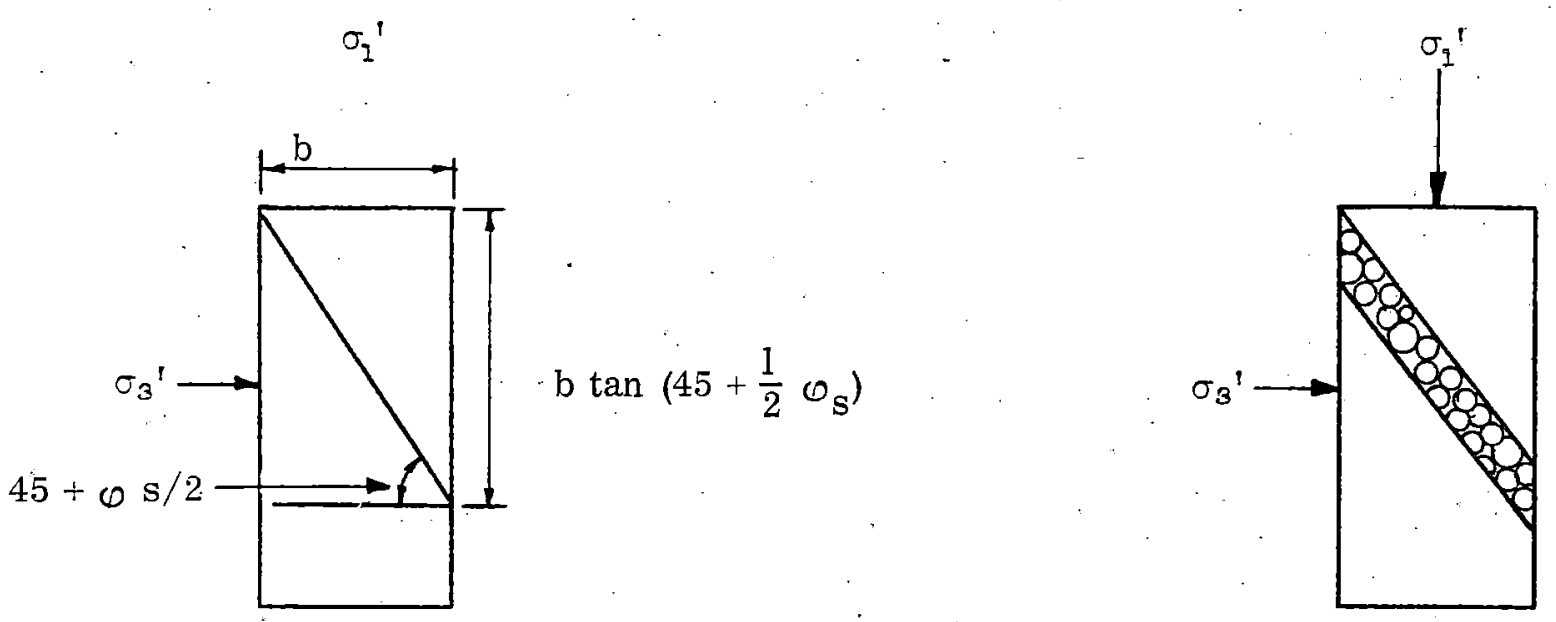

$$
\frac{\sigma_{1}^{\prime}}{\sigma_{3}{ }^{\prime}}=\tan ^{2}\left(45+\frac{1}{2} \cdot \phi_{s}\right)
$$

(a)

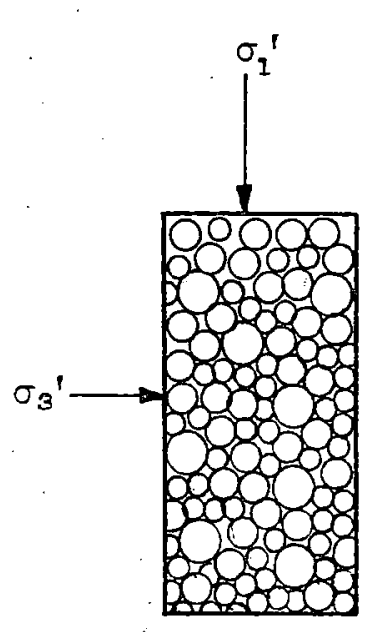

(c) $\quad \frac{1}{\sigma_{3}^{\prime}}=\Omega_{\mathrm{TCD}}$

(b)

Fig. 2. Physical model for each of the terms of Eq. (10g) (a) solid friction; (b) solid friction + frictional work due to dilatancy (c) dilatancy without friction. 
Consider the same assembly as shown in Fig. $2 c$ but filled with particles possessing a coefficient of solid friction $\mu=\tan \phi_{\mathrm{S}}$. The required ratio $\sigma_{1}{ }^{\prime} / \sigma_{3}{ }^{\prime}$ to produce relative movements between the particles where no sliding occurs between particles or groups of particles at a given instant is given by:

$$
\frac{\sigma_{1} !}{\sigma_{3}{ }^{\prime}}=\Omega_{\mathrm{TC}}+\Omega_{\mathrm{TCfD}}
$$

Similar analogies can be drawn in the case of Eqs. (10e) and (10f). Thus, the parameters $\Omega_{\mathrm{PD}}, \Omega_{\mathrm{TCD}}$, and $\Omega_{\mathrm{TED}}$ represent the threedimensional interference among the particles at a given instant. This three-dimensiona1 interference produces a rearrangement of the particles which is commonly known as interlocking. The effect of this three dimensional interference is not only to increase or decrease the rate of formation of sliding contacts, but also to increase or decrease the rate of volume change within the assembly.

The following quantities may be readily determined with a known value of $\Omega$ at any stage of the deformation process;

$$
\begin{aligned}
& \text { (a) Plane strain test } \\
& \frac{\delta W i_{\mathrm{D}}}{\sigma_{3}{ }^{\prime} \delta \epsilon_{1}}=\Omega_{\mathrm{PD}}+\frac{1}{2}\left(1+\frac{\sigma_{2}^{\prime}}{\sigma_{3}{ }^{\prime}}\right) \frac{\delta \mathrm{v}}{\delta \epsilon_{1}}, \\
& \frac{\delta W i_{E}}{\sigma_{3}{ }^{\prime} \delta \varepsilon_{1}}=\left(\frac{\sigma_{1}^{\prime}}{\sigma_{3}^{\prime}}-1\right)-\frac{\delta W i_{D}}{\sigma_{3}^{\prime} \delta \epsilon_{1}} \text {, } \\
& \frac{\delta W i_{D}}{\sigma_{1}{ }^{\prime} \delta \epsilon_{1}}=\frac{\delta W i_{D} / \sigma_{3}{ }^{\prime} \delta \epsilon_{1}}{\sigma_{1}{ }^{\prime} / \sigma_{3}{ }^{\prime}} \text {, } \\
& \frac{\delta W i_{f}}{\sigma_{1}{ }^{\prime} \delta \epsilon_{1}}=\frac{\delta W i_{f} / \sigma_{3}{ }^{\prime} \delta \epsilon_{1}}{\sigma_{1}{ }^{\prime} / \sigma_{3}{ }^{\prime}} \text {. }
\end{aligned}
$$


(b) Triaxial compression test

$$
\begin{aligned}
& \frac{\delta W i_{D}}{\sigma_{3}^{\prime} \delta \epsilon_{1}}=\Omega_{\mathrm{TCD}}+\frac{\delta \mathrm{V}}{\delta \epsilon_{1}}, \\
& \frac{\delta W i_{\mathrm{f}}}{\sigma_{3}^{\prime} \delta \epsilon_{1}}=\left(\frac{\sigma_{1}^{\prime}}{\sigma_{3}^{\prime}}-1\right)-\frac{\delta W i_{D}}{\sigma_{3}{ }^{\prime} \delta \epsilon_{1}}, \\
& \frac{\delta W i_{D}}{\sigma_{1}^{\prime} \delta \epsilon_{1}}=\frac{\delta W i_{D} / \sigma_{3}{ }^{\prime} \delta \epsilon_{1}}{\sigma_{1}^{\prime} / \sigma_{3}^{\prime}}, \\
& \frac{\delta W i_{f}}{\sigma_{1}^{\prime} \delta \epsilon_{1}}=\frac{\delta W i_{f} / \sigma_{3}^{\prime} \delta \epsilon_{1}}{\sigma_{1}^{\prime} / \sigma_{3}{ }^{\prime}} .
\end{aligned}
$$

(c) Triaxia1 extension test

$$
\begin{aligned}
& \frac{\delta W i_{D}}{\sigma_{3}{ }^{\prime} \delta \epsilon_{3}}=\Omega_{\text {TED }}+\frac{\sigma_{1}^{\prime}}{\sigma_{3}^{\prime}} \frac{\delta \mathrm{v}}{\delta \epsilon_{3}}, \\
& \frac{\delta W i_{f}}{\sigma_{3}^{\prime} \delta \epsilon_{3}}=\left(\frac{\sigma_{1}^{\prime}}{\sigma_{3}^{\prime}}-1\right)-\frac{\delta W i_{D}}{\sigma_{3}^{\prime} \delta \epsilon_{3}},
\end{aligned}
$$

where $\delta W i_{D}$ and $\delta W i_{f}$ are respectively the increments in internal work absorbed by the sample at a given instant in dilating or in friction; the quantities $\delta \mathrm{Wi}_{\mathrm{D}} / \sigma_{3}{ }^{\prime} \delta \epsilon_{1}$ and $\delta \mathrm{Wi}_{\mathrm{f}} / \sigma_{3}{ }^{\prime} \delta \epsilon_{1}$ are, respectively, the ratio of the increment in internal work absorbed in dilatancy or in friction to the product of the effective minor principal stress times the increment in natural axial strain at a given instant during the deformation process; and the quantities $\delta W i_{D} / \sigma_{1}^{\prime} \delta \epsilon_{1}$ and $\delta W i_{f} / \sigma_{1}^{\prime} \delta \epsilon_{1}$ are, respectively, the ratio of the increment in internal work absorbed in dilatancy or in friction to the increment in the work done on the simple by $\sigma_{1}^{\prime}$ at a given instant. The quantities $\delta W i_{D} / \sigma_{3}{ }^{\prime} \delta \epsilon_{1}$ and $\delta \mathrm{Wi}_{\mathrm{f}} / \mathrm{O}_{3}{ }^{\prime} \delta \epsilon_{1}$ do not represent a particular energy ratio, and they are selected as a convenient ratio for comparison of work components when $\delta \epsilon_{1}$ is applied and a constant effective minor principal stress is maintained. 


\section{TESTING OF THE THEORY}

Equations $(9 m),(9 n)$, and $(9 p)$ were tested against published data obtained by dependable research workers in the performance of plane strain, triaxial compression, and extension tests on cohesionless soils.

There are two unknowns, $\sin \phi$ and $\Omega$, in each of the Eqs. (9m), $(9 n)$, and $(9 p)$. Plots of the experimental published data according to Eqs. $(9 m),(9 n)$, and $(9 p)$ showed a straight line form over a wide deformation range; and thus allowed the determination of $\sin \phi_{S}$ and $\Omega$.

The values of the effective major, intermediate, and minor principal stresses; the change in volume in percent of either the initial or the actual sample volume; and the axial strain in percent of either the initial or the actual height of the sample, were obtained from the pub1ished test data.

Although identical notation was used, the value of $\delta v / \delta \varepsilon_{1}$ was based on either the "engineering" volumetric and axial strains or the "natural" volumetric and axial strains. Whereas the "engineering" volumetric and axial strains were given in percent of the initial volume and height of the sample, respectively, the "natural" volumetric and axial strains were given in percent of the actual volume and height, respectively, of the sample at that instant. The correct value of $\delta v / \delta \epsilon_{1}$ for use in Eqs. (9m), (9n), and (9p) is the ratio of the increments in "natural" strains. However, the differences between the two ratios are small unless the deformation exceeds about 5 percent of the axial strain. The value of the engineering axial strain in 
percentage is smaller than the corresponding value of the natural axial strain in percentage for large axial deformation. The difference did not influence the value of $\sin \phi_{s}$, but it may have a slight effect on the values of the parameter $\Omega$ corresponding to the curve after the maximum value of $\sigma_{1}{ }^{\prime} / \sigma_{3}{ }^{\prime}$ is achieved within the sample.

Cornforth (1964) performed plane strain tests on a river sand from Brasted, Kent, in England. The values of the effective major, intermediate and minor principal stress, the "engineering" volumetric and axial strains in percent, and the initial porosity were obtained (Fig. 10, Cornforth, 1964). The values of $\sigma_{1}^{\prime} / \sigma_{3}^{\prime}-1-\frac{1}{2}(1+$ $\left.\sigma_{2}{ }^{\prime} / \sigma_{3}{ }^{\prime} \delta v / \delta \epsilon_{1}\right)$ were plotted against the values of $\sigma_{1}{ }^{\prime} / \sigma_{3}{ }^{\prime}+1+\frac{1}{2}$ $\left(1+\sigma_{2}^{\prime} / \sigma_{3}^{\prime}\right) \delta \mathrm{v} / \delta \varepsilon_{1}$ in Fig. 3. All the points corresponding to lower values of $\sigma_{1}{ }^{\prime} / \sigma_{3}{ }^{\prime}$ than the maximum value of $\sigma_{1}{ }^{\prime} / \sigma_{3}{ }^{\prime}$ plot on a straight line (solid line, Fig. 3) which thus allowed the determination of $\sin \phi_{S}$ and $\Omega_{P}$. The values of $\sin \phi_{S}$ and $\Omega_{P}$ are 0.408 and 0.800 , respectively. Thus the value of the solid friction angle of the Brasted sand is 24.1 degrees. Figure 3 also shows a dashed line which corresponds to values of $\sigma_{1}{ }^{\prime} / \sigma_{3}{ }^{\prime}$ obtained after the maximum value of $\sigma_{1}{ }^{\prime} / \sigma_{3}{ }^{\prime}$ was reached.

Barden and Khayatt (1966) performed triaxial extension tests on a sand which they denoted as River Welland sand. The values of $\frac{\sigma_{1}^{\prime}}{\sigma_{3}^{\prime}}$ $\frac{1}{1-\delta \mathrm{v} / \delta \varepsilon_{3}}$ were plotted against the values of $\frac{\sigma_{1}{ }^{\prime}}{\sigma_{3}{ }^{\prime}}+\frac{1}{1-\delta \mathrm{v} / \delta \varepsilon_{3}}$ in Fig. 4 . The relationship given by Eq. (9p) is a straight line as shown in Fig. 4 and the calculated values of $\sin \phi_{S}$ and $\Omega_{\mathrm{TE}}^{\prime}$ are, respectively 0.413 and 0.260 . The value of the angle of solid friction for this sand is 24.4 degrees. 


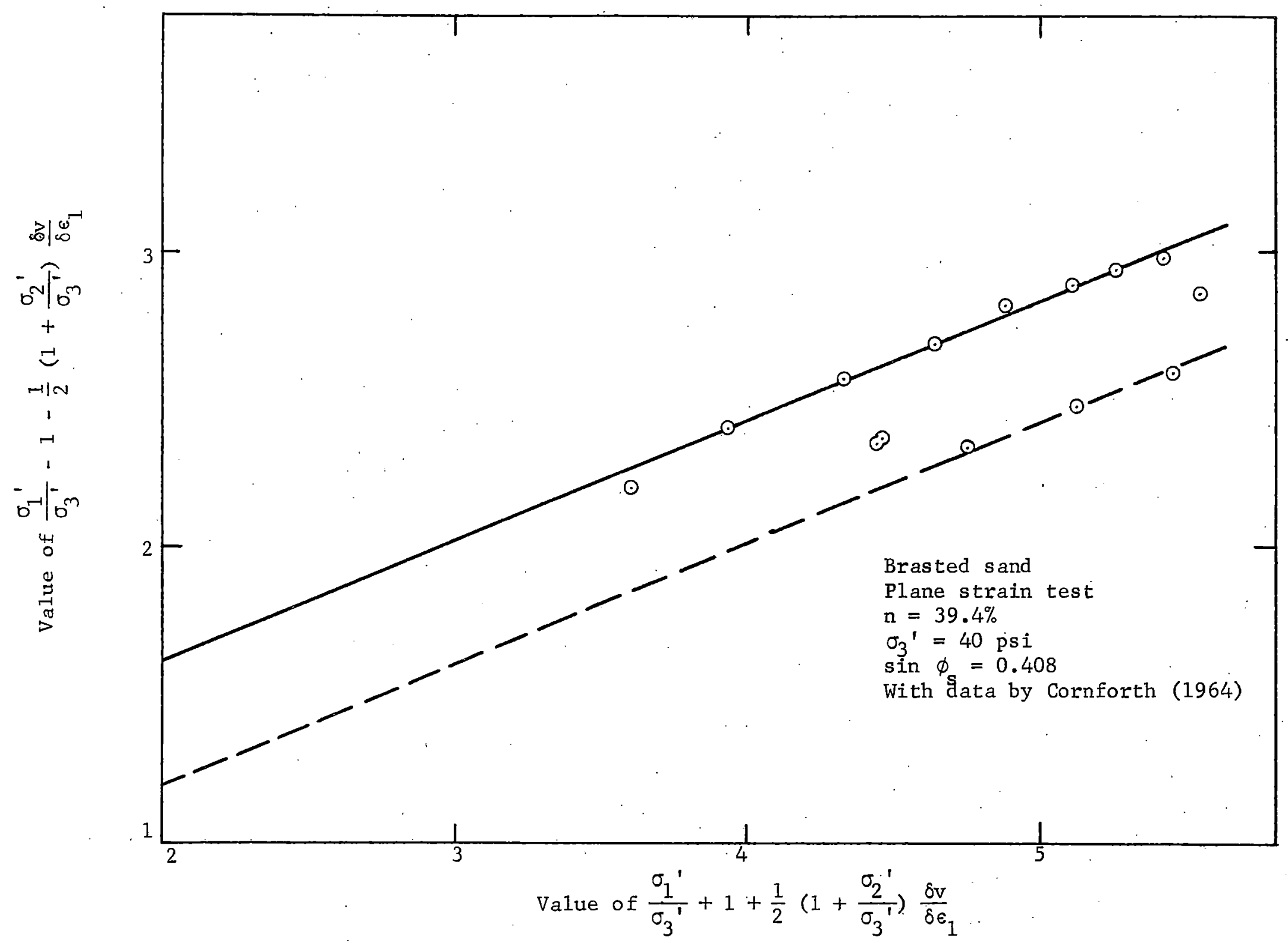

Fig. 3. Testing of Eq. (9m). 


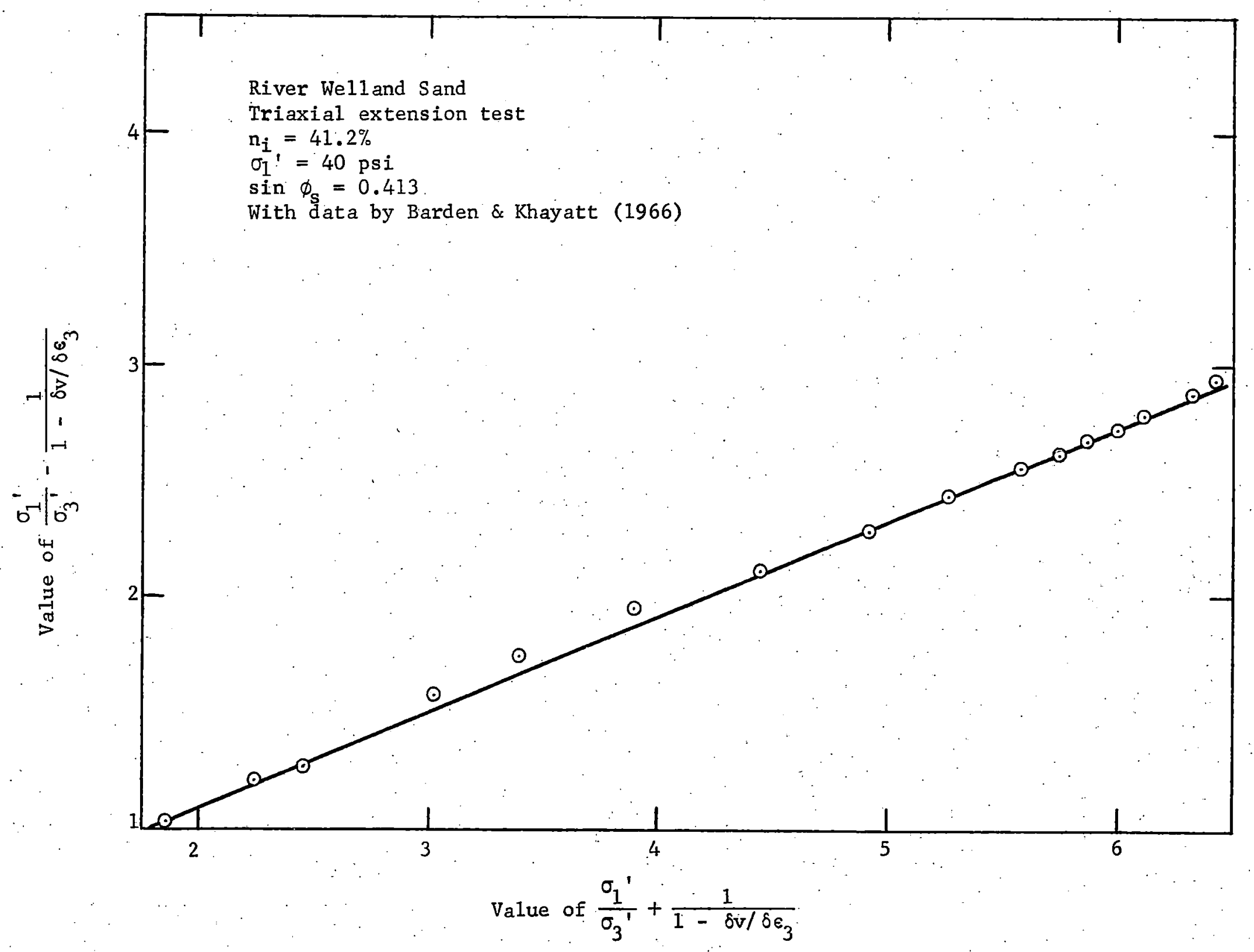

Fig. 4. Testing of Eq. (9p). 
The values of $\sigma_{1}{ }^{\prime} / \sigma_{3}{ }^{\prime}-\left(1+\delta v / \delta \varepsilon_{1}\right)$ were plotted against the values of $\sigma_{1}{ }^{\prime} / \sigma_{3}{ }^{\prime}+\left(1+\delta v / \delta \epsilon_{1}\right)$, obtained from published triaxial. compression test data, in Figs. 5 to 13. The relationship expressed by Eq. (9n) is also a straight line for all the data presented.

A summary of the published data shown in Figs. 3 to 13 is presented in Table 1, together with the values of the angle of solid friction and of the parameter $\Omega$ as calculated from the corresponding Eqs. (9m), (9n), and $(9 \mathrm{p})$.

It is apparent from Table 1 that the angle of solid friction of the sands varies between 24 to 24.5 degrees and these values are independent of the initial void ratio, the type of shear test performed on the sand, and the level of the confining pressure within the tested range. Deviations are acknowledged in Table 1 for the older published data, which may be considered less reliable since many refinements have been introduced in testing techniques.

The main mineral component of these sands is quartz. Horn (1961) using a special technique measured the coefficient of friction between two highly polished surfaces of pure quartz. Horn's measured values for quartz under submerged conditions varied between 0.42 and 0.51 , which correspond to angles of solid friction between 22.8 and 27.0 degrees. The purpose in bringing forward Horn's results in pure quartz is solely for comparison and not to support the correctness of the values obtained by the proposed equations. In fact, Horn's. results in feldspar correspond to a solid friction angle of 37 degrees, which differs from the value of 32.7 degrees appearing in Table 1. The independent evaluation of the coefficient of friction 
Table 1. Summary of selected published data.

\begin{tabular}{|c|c|c|c|c|c|c|c|}
\hline $\begin{array}{l}\text { With } \\
\text { data } \\
\text { by }\end{array}$ & Figure & $\begin{array}{l}\text { Granular } \\
\text { material }\end{array}$ & $\begin{array}{c}\text { Type } \\
\text { of } \\
\text { test }\end{array}$ & $\begin{array}{l}\text { Initial } \\
\text { porosity } \\
\quad(\%)\end{array}$ & $\begin{array}{l}\text { Confining } \\
\text { pressure } \\
\quad \text { (psi) }\end{array}$ & $\begin{array}{r}\text { Values } \\
\text { Eqs } \\
(9 \mathrm{~m})(9 \mathrm{n}) \\
\frac{\phi_{\mathrm{s}}}{\text { (degree) }}\end{array}$ & $\begin{array}{l}\text { from } \\
\quad(9 \mathrm{p}) \\
\Omega\end{array}$ \\
\hline $\begin{array}{l}\text { Cornforth } \\
\text { (1964) }\end{array}$ & 3 & s and & $\begin{array}{l}\text { plane } \\
\text { strain }\end{array}$ & 39.4 & 40 & 24.1 & $\begin{array}{c}0.800 \\
.\end{array}$ \\
\hline $\begin{array}{l}\text { Barden \& } \\
\text { Khayatt } \\
(1966)\end{array}$ & 4 & s and & $\begin{array}{l}\text { triaxial } \\
\text { extension }\end{array}$ & 41.2 & 40 & 24.4 & 0.260 \\
\hline $\begin{array}{l}\text { Barden \& } \\
\text { Khayatt } \\
(1966)\end{array}$ & 5 & s and & $\begin{array}{l}\text { triaxial } \\
\text { compression }\end{array}$ & 39.8 & 40 & 24.4 & 0.331 \\
\hline $\begin{array}{l}\text { Barden \& } \\
\text { Khayatt } \\
(1966)\end{array}$ & 6 & s and & $\begin{array}{l}\text { triaxial } \\
\text { compression. }\end{array}$ & 40.3 & 40 & 24.6 & 0.260 \\
\hline $\begin{array}{l}\text { Taylor } \\
\text { (1948) }\end{array}$ & 7 & $s$ and & $\begin{array}{l}\text { triaxial } \\
\text { compression }\end{array}$ & 37.7 & 30 & 22.3 & 0.888 \\
\hline $\begin{array}{l}\text { Lambe } \\
\text { (1961) }\end{array}$ & 8 & s and & $\begin{array}{l}\text { triaxial } \\
\text { compression }\end{array}$ & 31.1 & 30 & 22.5 & 0.785 \\
\hline $\begin{array}{l}\text { Bishop } \& \\
\text { Green (1965) }\end{array}$ & 9 & s and & $\begin{array}{l}\text { triaxial } \\
\text { compression }\end{array}$ & 41.4 & 40 & 24.1 & 0.395 \\
\hline $\begin{array}{l}\text { Bishop \& } \\
\text { Green (1965) }\end{array}$ & 10 & s and & $\begin{array}{l}\text { triaxial } \\
\text { compression }\end{array}$ & 41.5 & 40 & 24.1 & 0.455 \\
\hline $\begin{array}{c}\text { Penman } \\
(1953)\end{array}$ & 11 & silt & triaxial & 36.0 & 100 & 26.9 & 0.600 \\
\hline $\begin{array}{l}\text { Lee } \\
(1966)\end{array}$ & 12 & feldspar & $\begin{array}{l}\text { triaxial } \\
\text { compression }\end{array}$ & 35.0 & 30 & 32.7 & 0.390 \\
\hline $\begin{array}{l}\text { Lee } \\
\text { (1966) }\end{array}$ & 13 & feldspar & $\begin{array}{l}\text { triaxial } \\
\text { compression }\end{array}$ & 34.9 & 60 & 32.7 & 0.435 \\
\hline
\end{tabular}




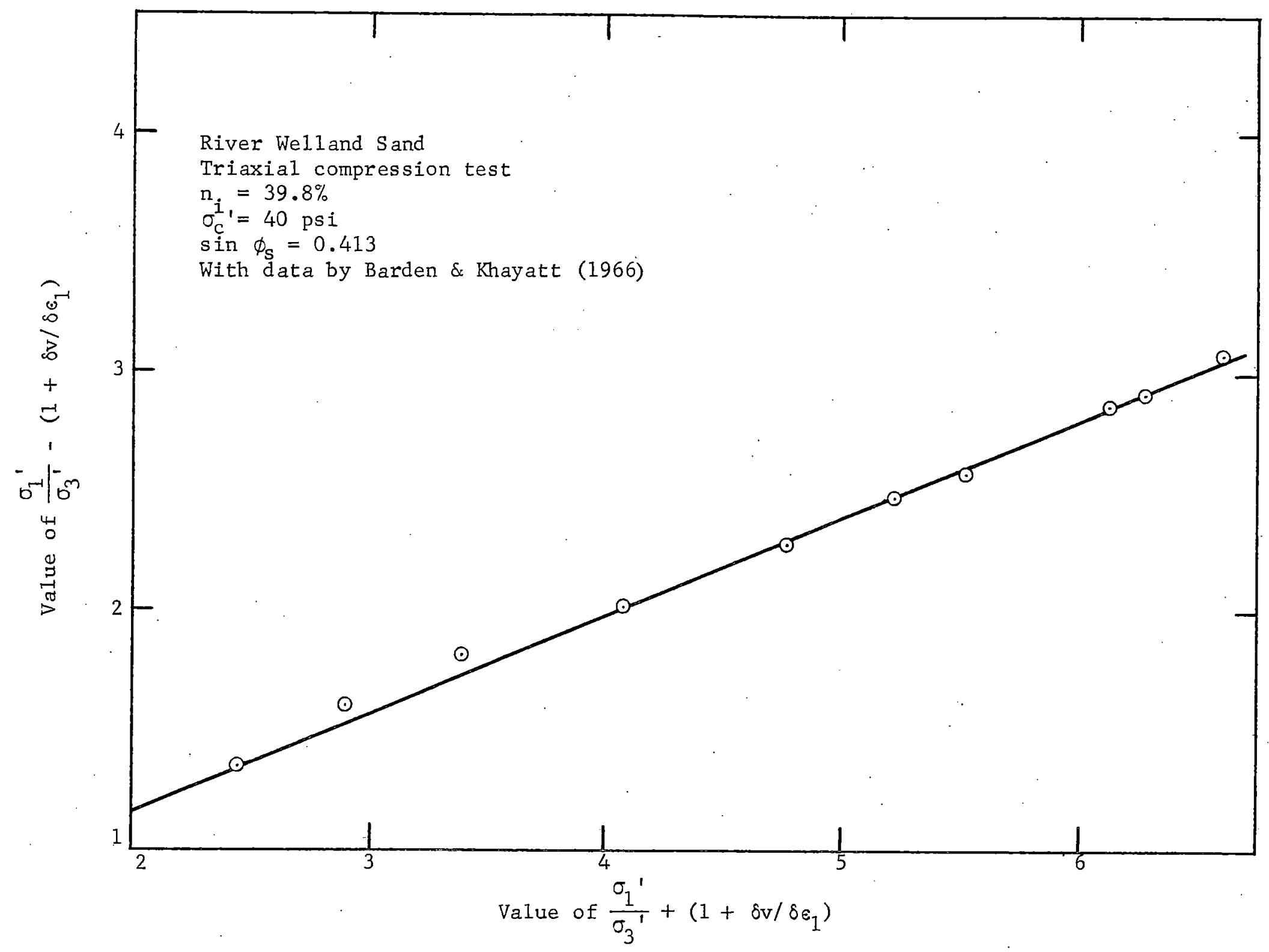

Fig. 5. Testing of Eq. (9n). 


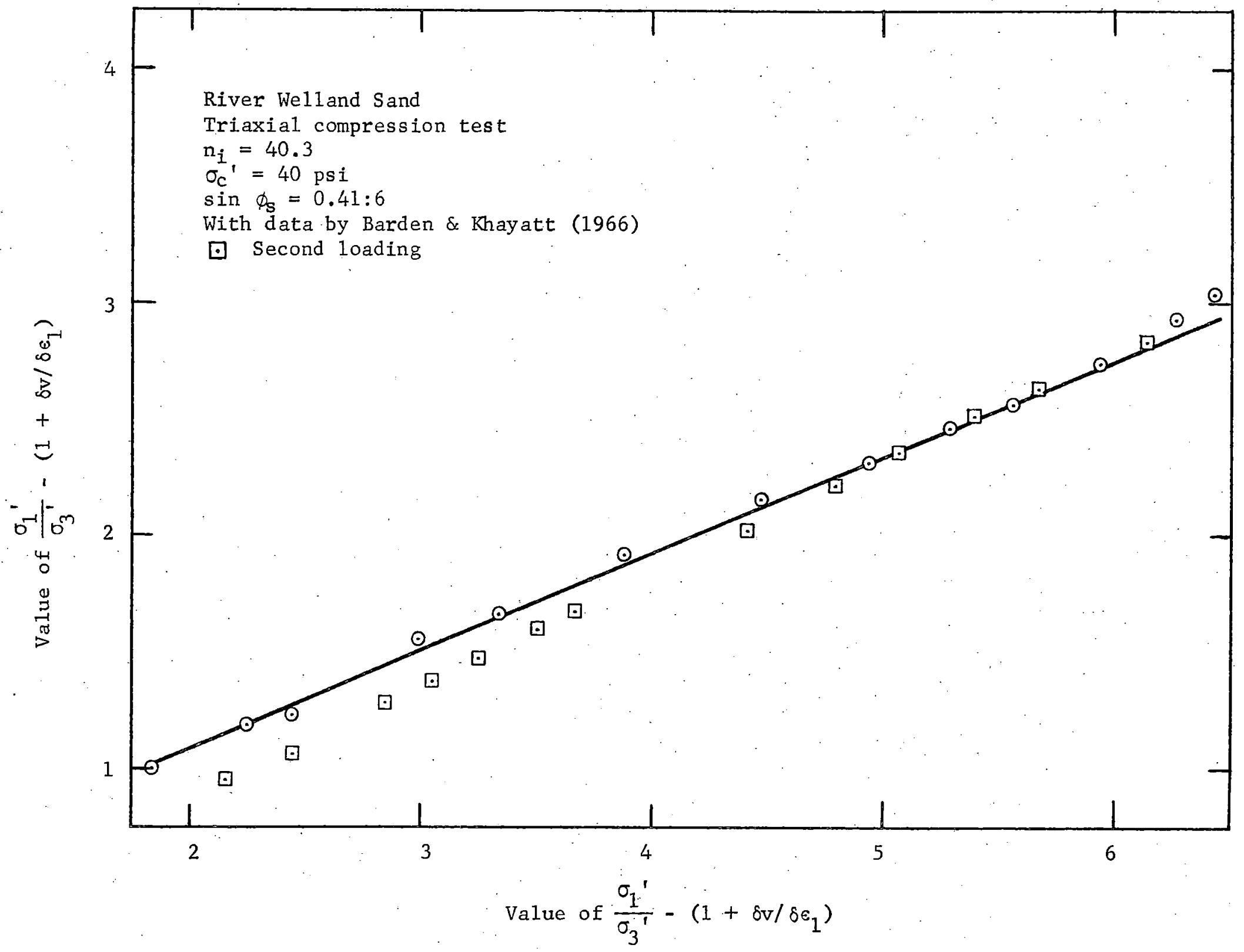

Fig. 6. Testing of Eq. (9n): 


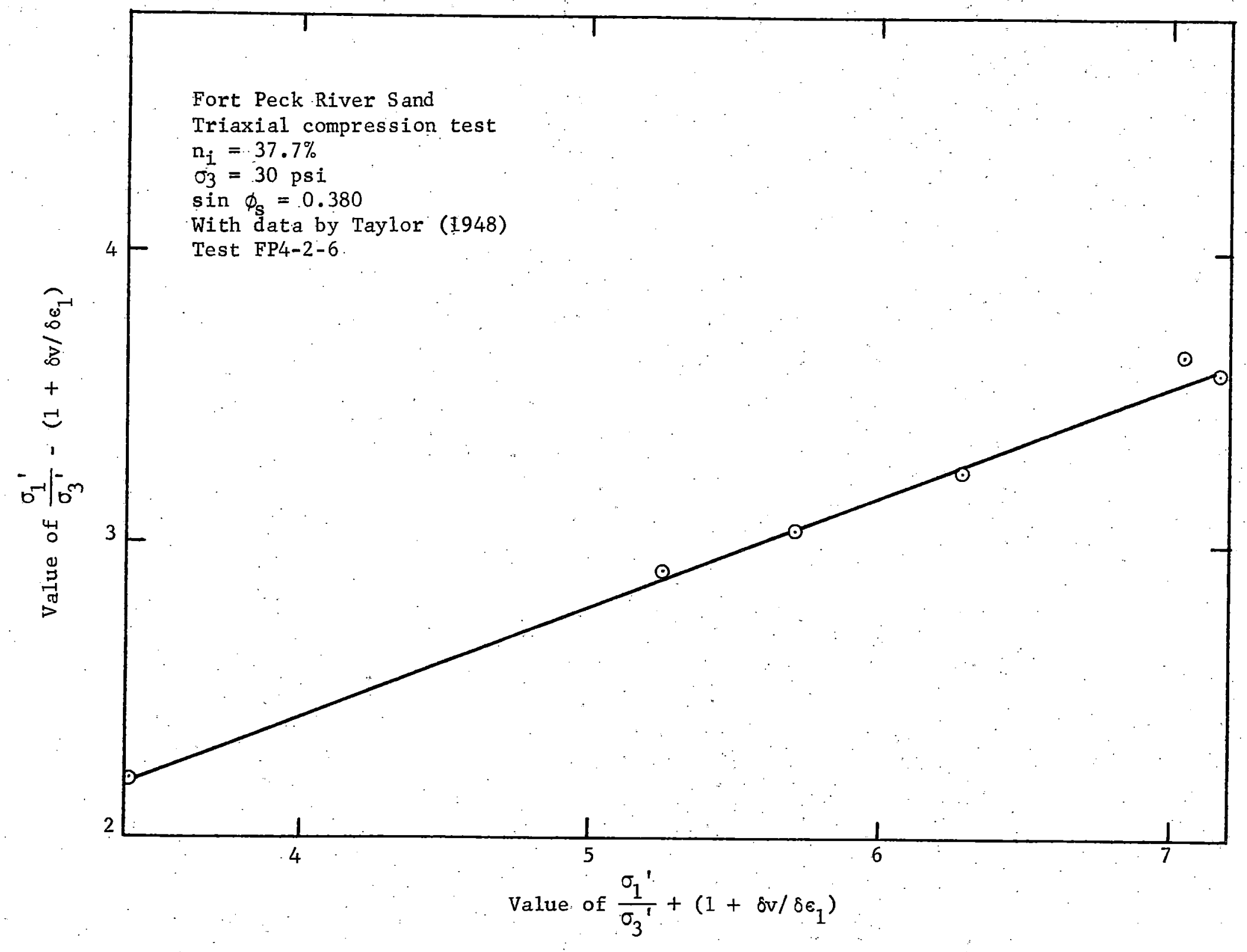

Fig. 7. Testing of Eq. (9n). 


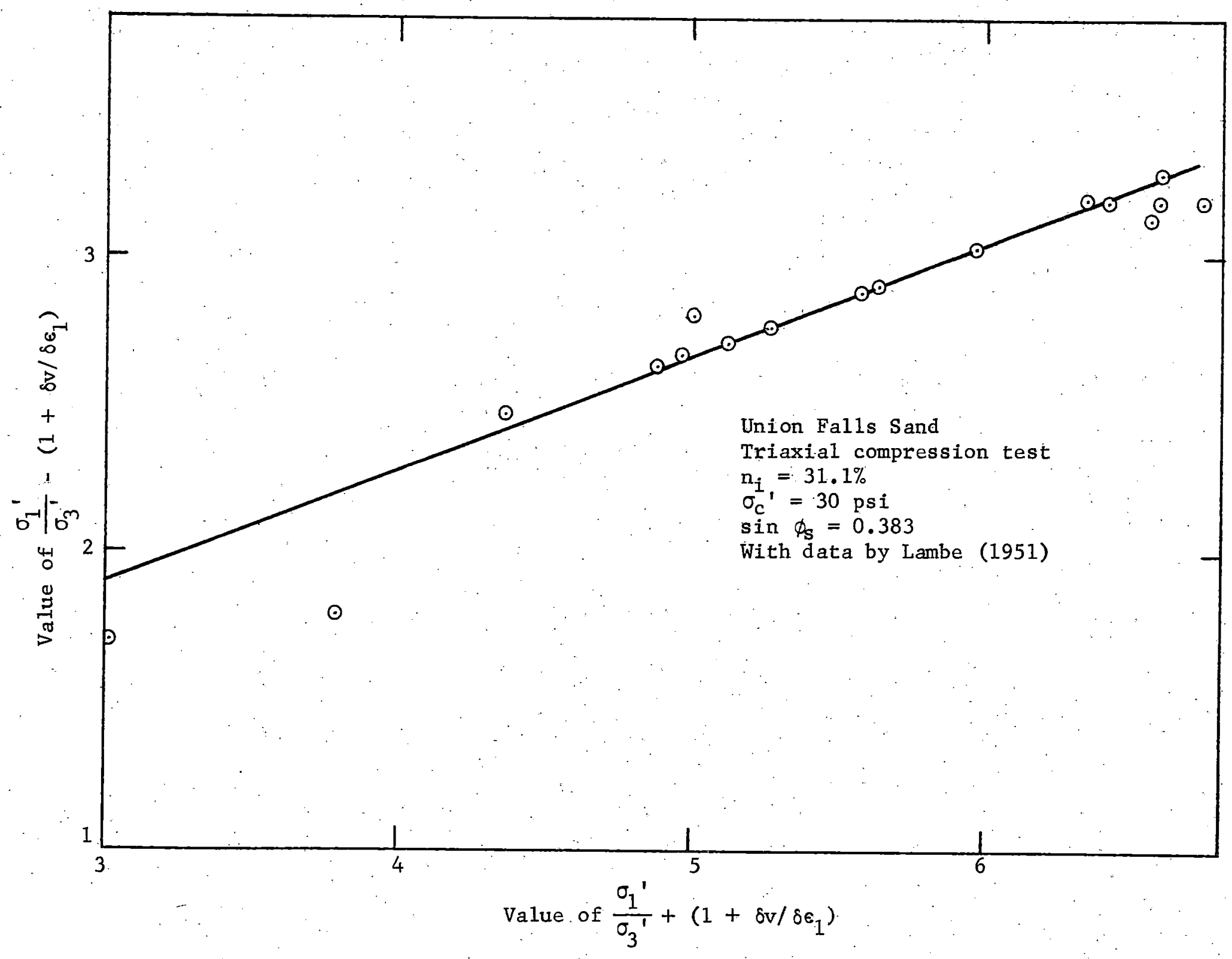

Fig. 8. Testing of Eq. (9n). 


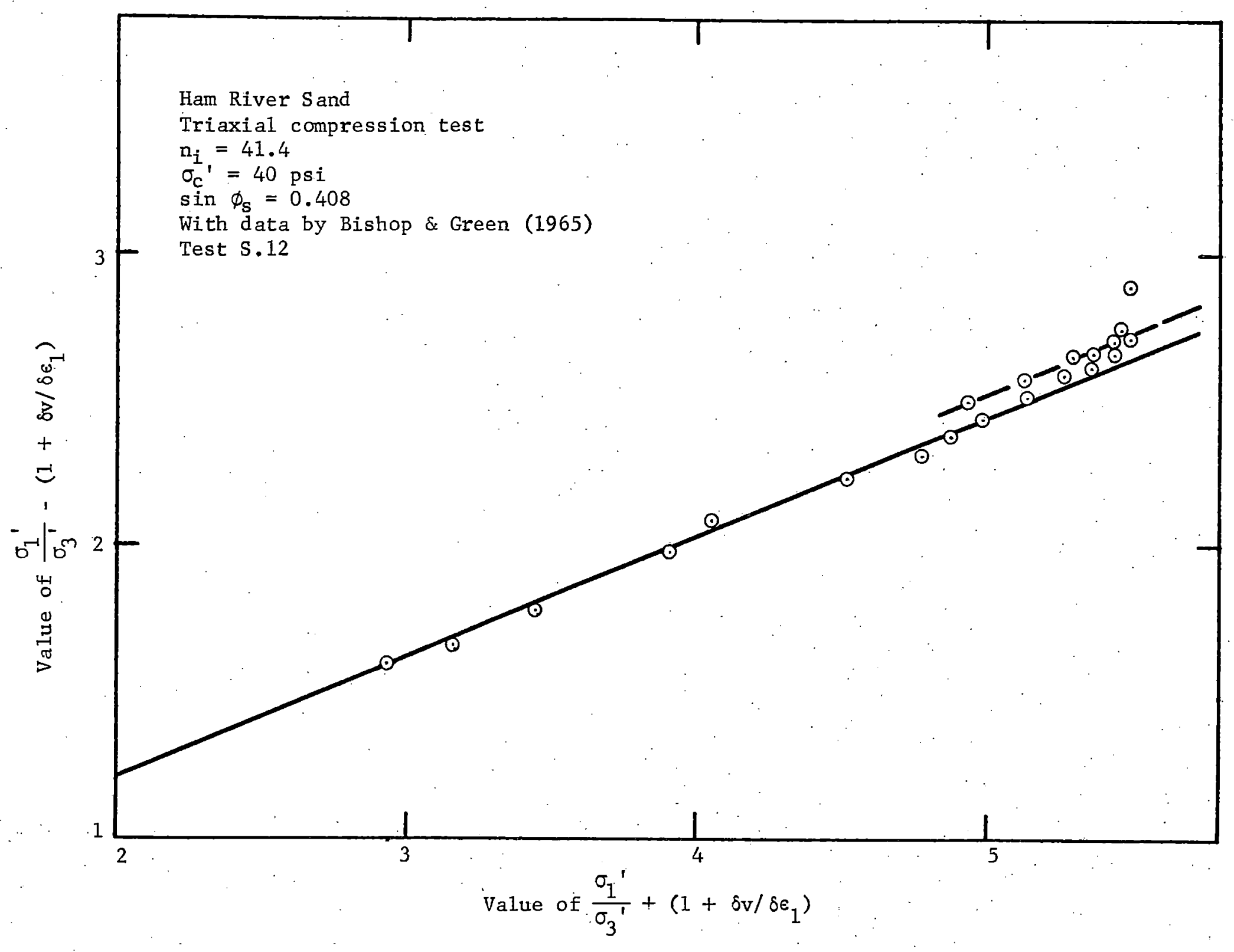

s

Fig. 9. Testing of Eq. (9n). 


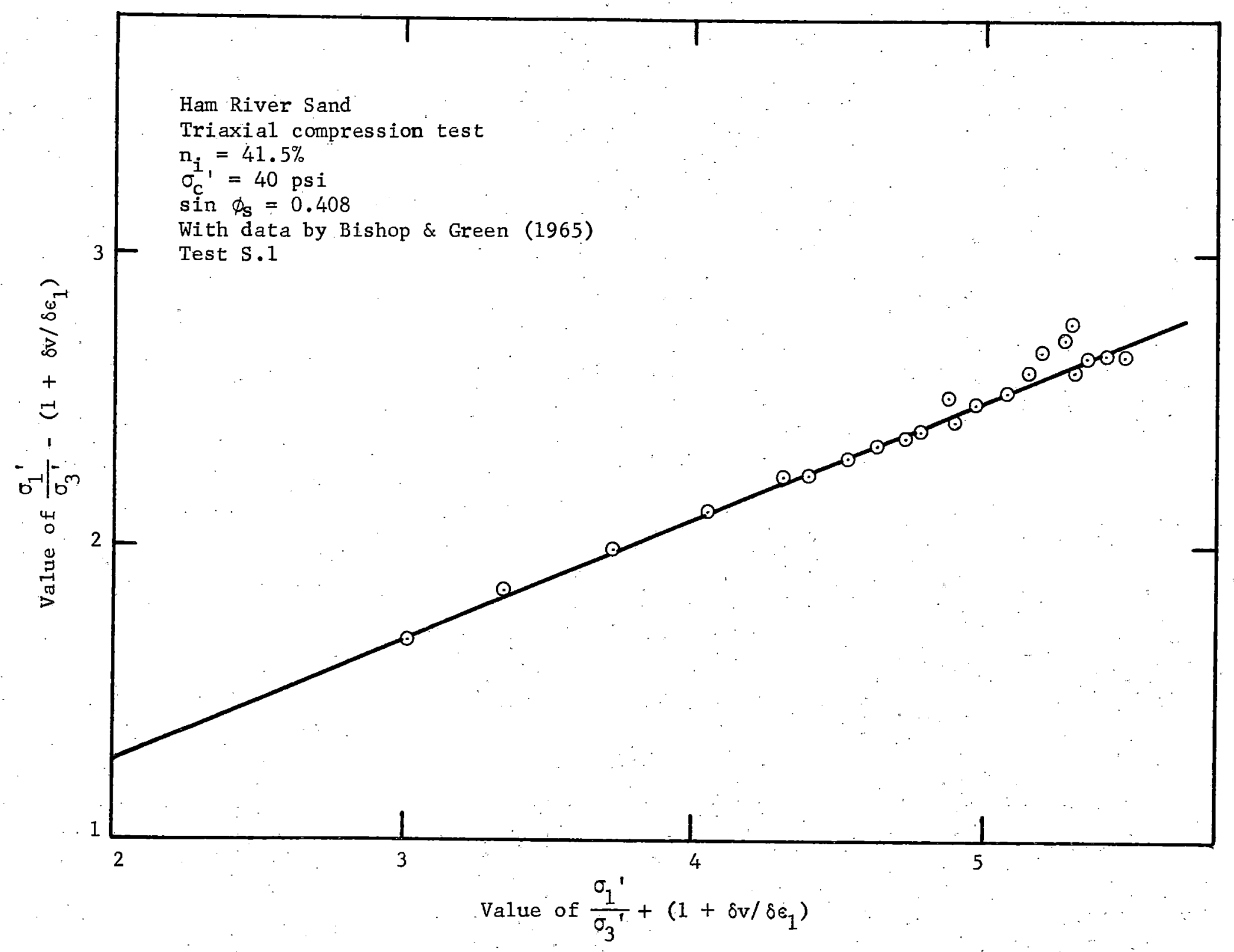

Fig. 10. Testing of Eq. (9n). 


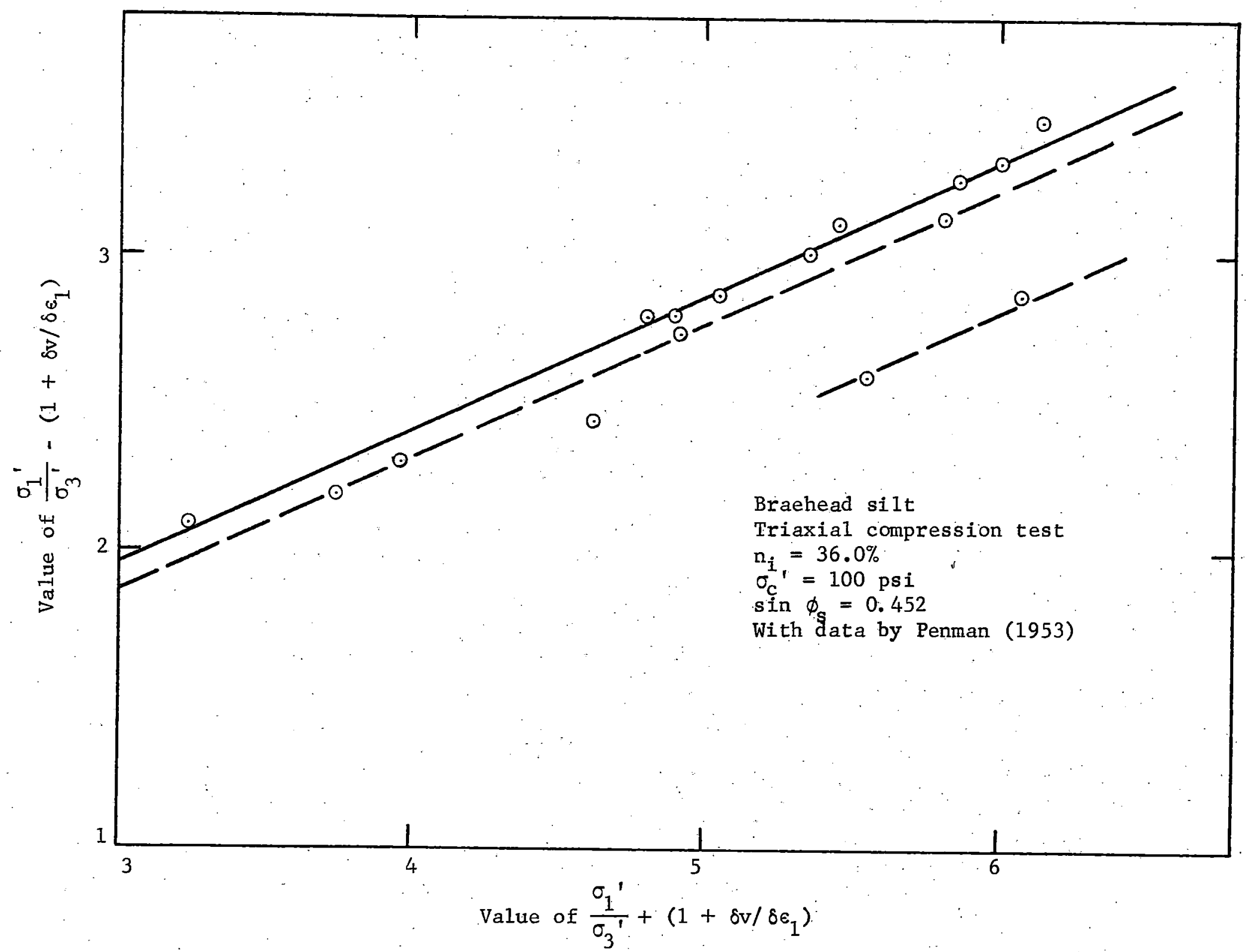

Fig. 11. Testing of Eq. (9n). 


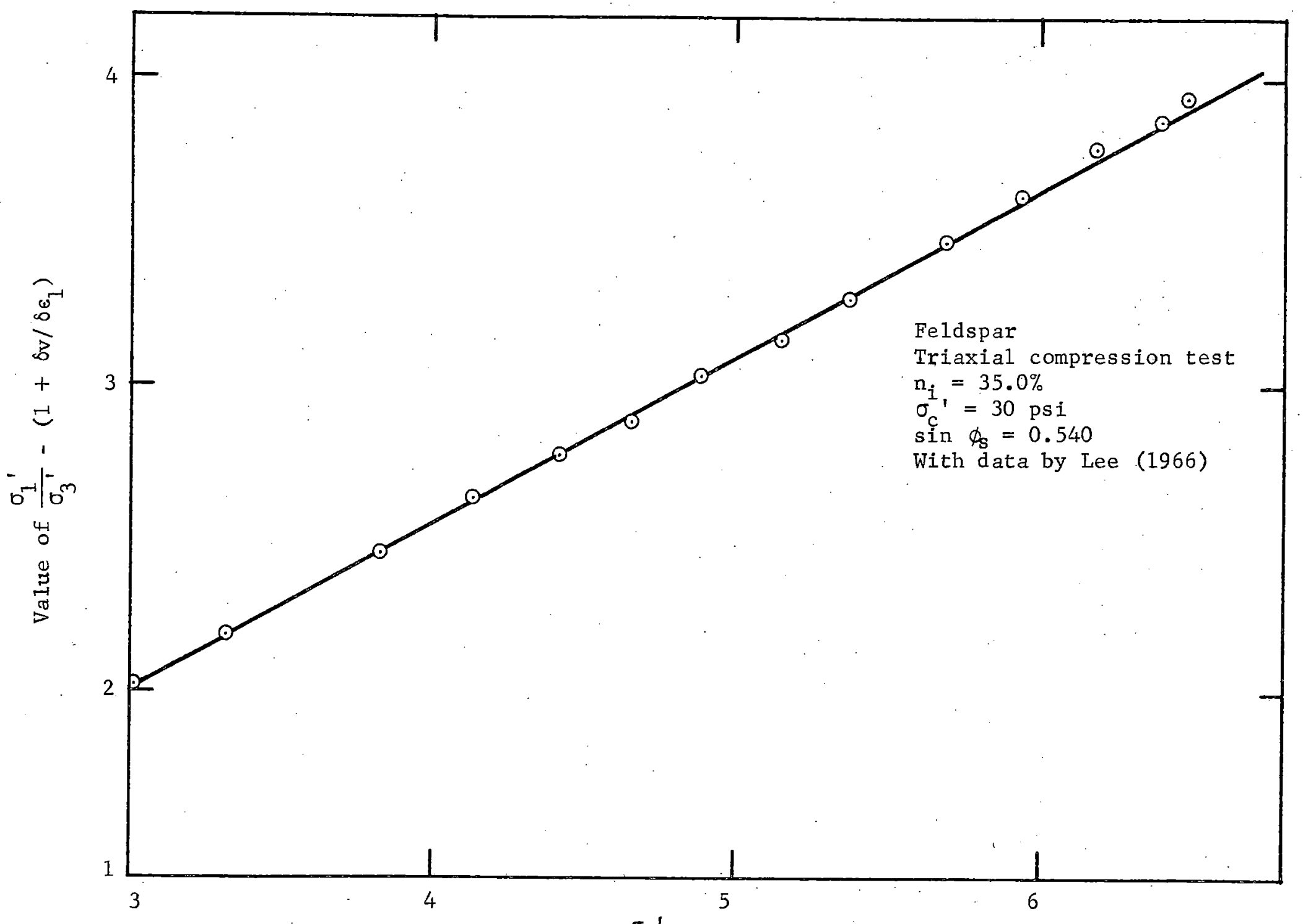

Value of $\frac{\sigma_{1}^{\prime}}{\sigma_{3}^{\prime}}+\left(1+\delta \mathrm{v} / \delta \epsilon_{1}\right)$

Fig. 12. Testing of Eq. (9n). 


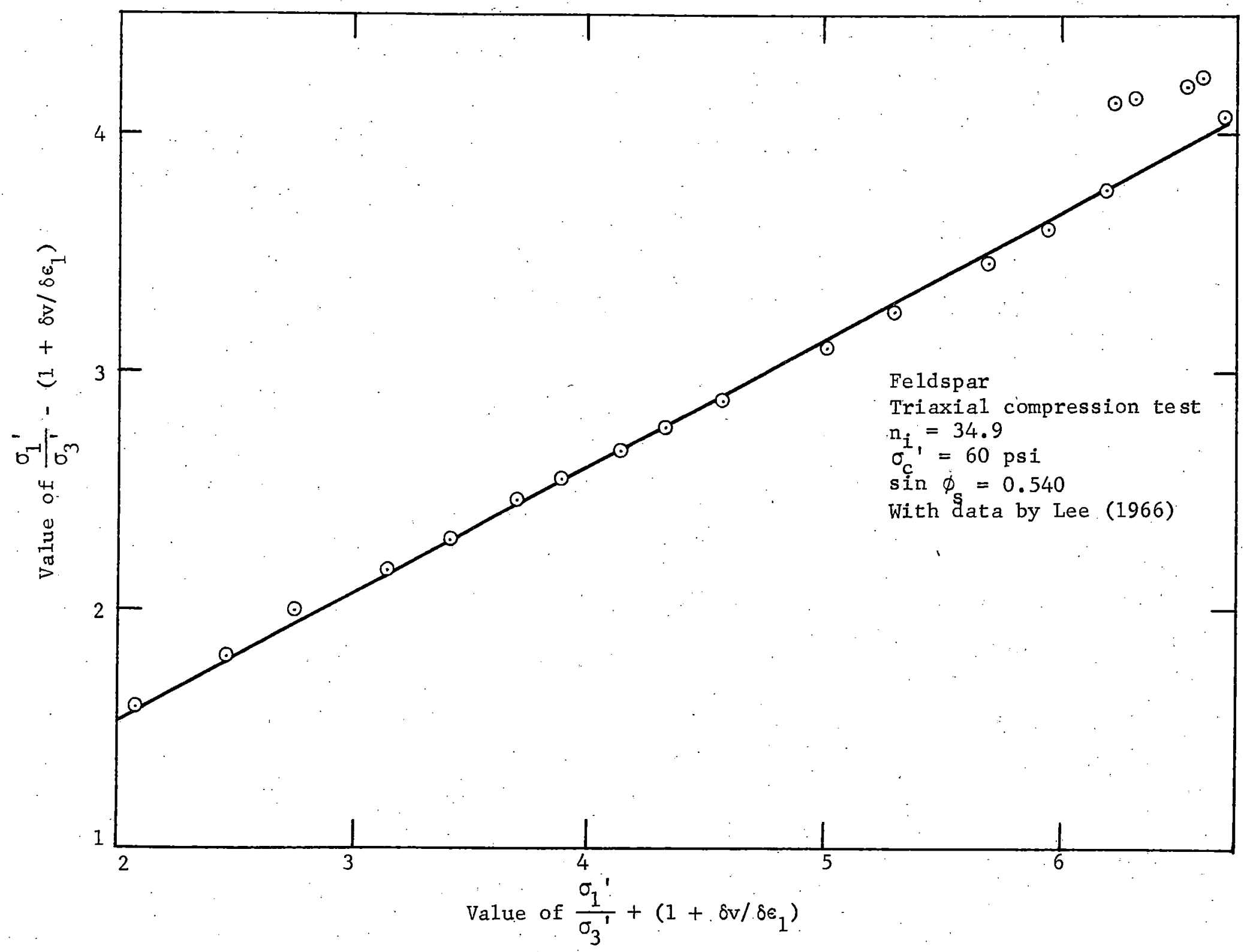

Fig. 13. Testing of Eq. (9n). 
of minerals is a helpful guide to what values may be expected but since soils vary widely in mineralogical composition, particularly of grain surfaces, their coefficient of friction may be expected to deviate from the value of the main mineral component.

The values of the parameter $\Omega$ for plane strain are considerably higher than for triaxial compression and extension tests; this is a direct result of a larger amount of interlocking or three-dimensional interference among the particles due to the imposed strain conditions, namely no strain is being allowed in the direction of the intermediate principal stress.

The parameter $\Omega$ is a function of the initial porosity, the level of the confining pressure and the gradation. However, the calculated values of $\Omega$ from two tests, Figs. 9 and 10, with data from Bishop and Green (1965) appearing in Table 1 are different in spite of both samples being at the same initial porosity and confining pressure. These samples were, however, tested with different boundary conditions. The sample with the higher value of the parameter $\Omega$ was tested with "fixed" ends whereas the other sample was tested with "frictionless" ends. The term "fixed" ends indicates that friction was developed between base and cap and the ends of the sample, whereas "frictionless" ends indicate a reduction of that friction to a minimum. The effect of "fixed" ends is to increase the value of the parameter $\Omega$ during the pre-peak deformation range where "peak" denotes the maximum value of the ratio $\sigma_{1}^{\prime} / \sigma_{3}{ }^{\prime}$. This increase is explained by reduced rate of volume change and by an increased slope of the $\sigma_{1}{ }^{\prime} / \sigma_{3}{ }^{\prime}$ versus axial strain curve, as a result of which the peak value is reached at smaller 
strains in the sample tested with "fixed" ends (Bishop and Green, 1965). "Fixed" ends also modified the post-peak behavior: The rate of decrease in the values of $\sigma_{1}{ }^{\prime} / \sigma_{3}{ }^{\prime}$ with respect to axial strain is higher than in the sample with frictionless ends (Bishop and Green, 1965). The effect of this higher rate of decrease reduces the value of the parameter $\Omega$ in comparison with the other sample, where post-peak values of $\Omega$ are higher than their pre-peak values, as shown in Fig. 9 by the upper dashed line. Similar higher values of $\Omega$ for post-peak values of $\sigma_{1}^{\prime} / \sigma_{3}^{\prime}$ are shown in Fig. 13 with data by Lee (1966) on feldspar tested with frictionless ends.

The high calculated values of $\Omega$ for the tests with data by Taylor (1948) and Lambe (1951) are a result of the effect of restrained ends on the sample's deformational behavior, and also to probable errors in the measurement of the applied normal loads due to piston friction. Values of the functions $F, F^{\prime}$, and $D$ with respect to the axial strain are plotted in Fig. 14 with data by Cornforth (1964). The functions $F, \mathrm{~F}^{\mathrm{I}}$, and $\mathrm{D}$ are defined by Eqs. (12a), (12b), and (12c) respectively:

$$
\text { Let } \begin{aligned}
F & =\frac{\delta W i_{f}}{\sigma_{3}{ }^{\prime} \delta \epsilon_{1}}, \\
F^{\prime} & =\frac{\delta W i_{f}}{\sigma_{3} ! \delta \epsilon_{1}}+\Omega_{f}, \\
\text { and } \quad D & =\frac{\delta W i_{f}}{\sigma_{3}{ }^{\prime} \delta \epsilon_{1}}+\Omega_{f}+\Omega .
\end{aligned}
$$

The plot of the values of $F$ against axial strain represents the changes in the rates of absorption of internal work by friction as the test progresses. The values of $\dot{F}^{\prime}$ plotted against the axial strain represent 


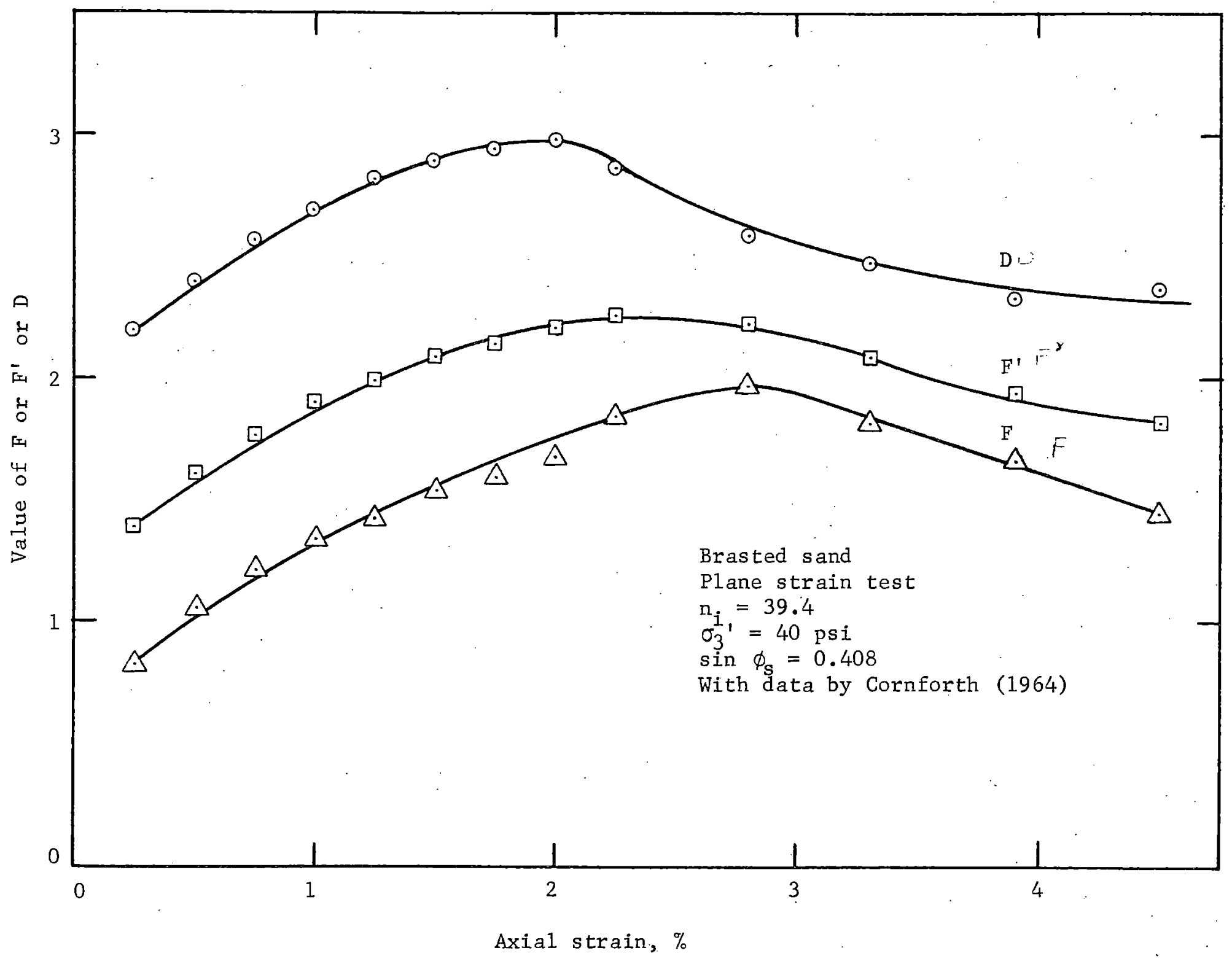

Fig. 14. Value of the functions $F, F^{\prime}$, and $D$ versus axial strain. 
these changes plus additional internal work absorbed in friction due to particle interference. The values of $D$ plotted against the axial strain represent these changes plus the work absorbed in dilatancy, and in effect show changes in the rates of the total internal work corrected for the work done by the sample against the applied stresses.

An interesting feature of Fig. 14 is the amount of strain required to achieve the maximum values of $F$ and $D$ within the sample. The maximum value of the function $D$ occurs at this peak value, and after the peak it dropped rapidly. This indicates that the sample showed maximum interference between particles at the peak. Also to bring about the highest possible number of sliding contacts within the sample, this maximum interference is broken up, as shown by the increase in $F$. After the maximum value of $F$ has been achieved, $F$ decreases at a faster rate than $D$, indicating that the structure of the sample is now in a looser state.

Similar features are shown in Fig. 15 with data by Lambe (1951). However, there'is less difference in the amount of strain required for $F$ and $D$ to develop their respective maximum. This may be explained by the effect of end restraint on the deformational behavior of the sample. That is, end restraint apparently decreases the "free". dilatant volume, and changes the stress distribution within this "free" dilatant volume where the term "free" volume refers to that part of the sample unaffected by end restraint.

Figure 16 is a plot of the values of $\delta W i_{D} / \sigma_{1}{ }^{\prime} \delta \epsilon_{1}, \delta W i_{f} / \sigma_{1}{ }^{\prime} \delta \epsilon_{1}$, and $\delta W i / \sigma_{1}{ }^{\prime} \delta \epsilon_{1}$ versus the axial strain for a plane strain test with data by Cornforth (1964). The important feature of this graph is the 


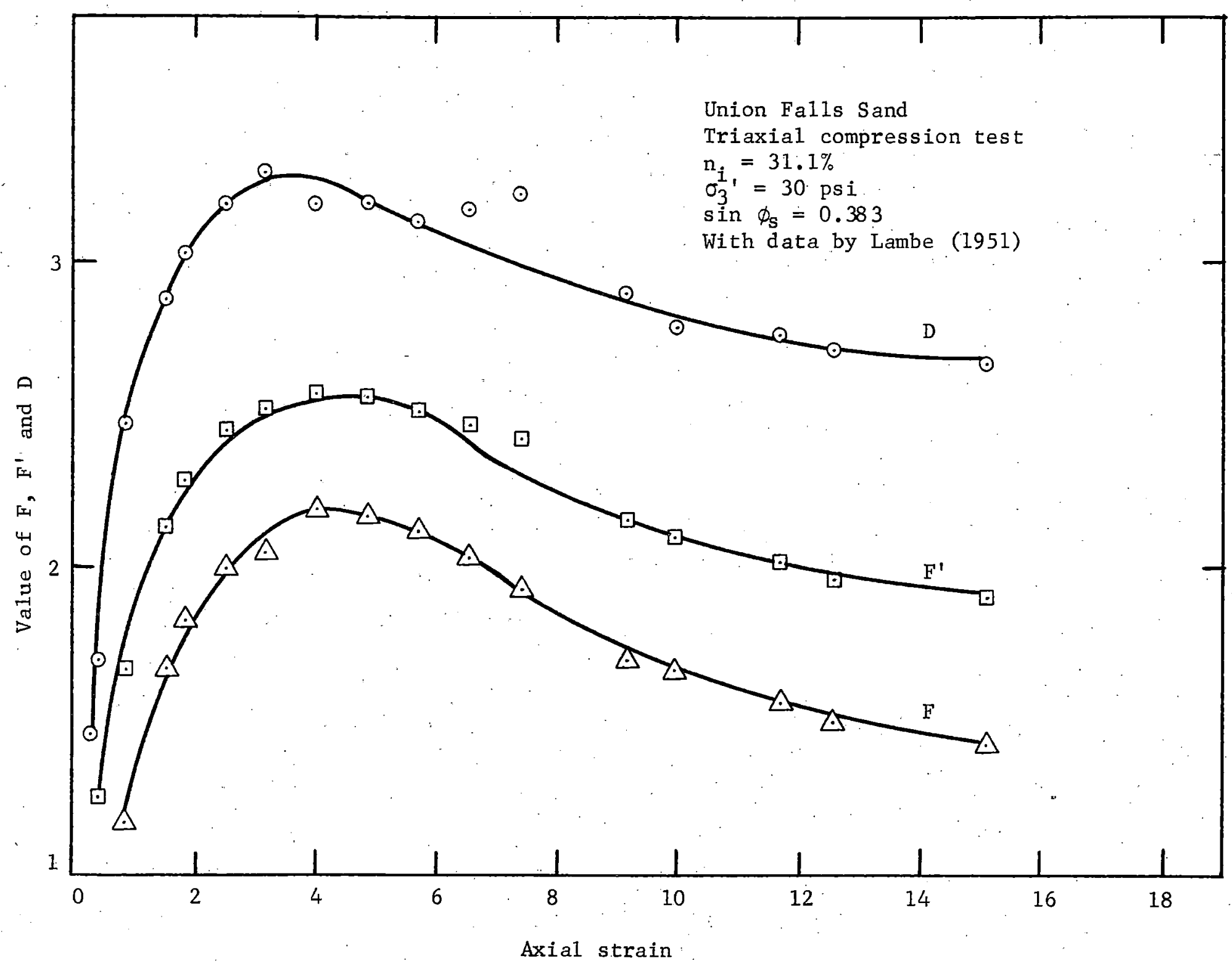

Fig: 15. Value of the functions $F, F^{\prime}$, and $D$ versus axial strain. 


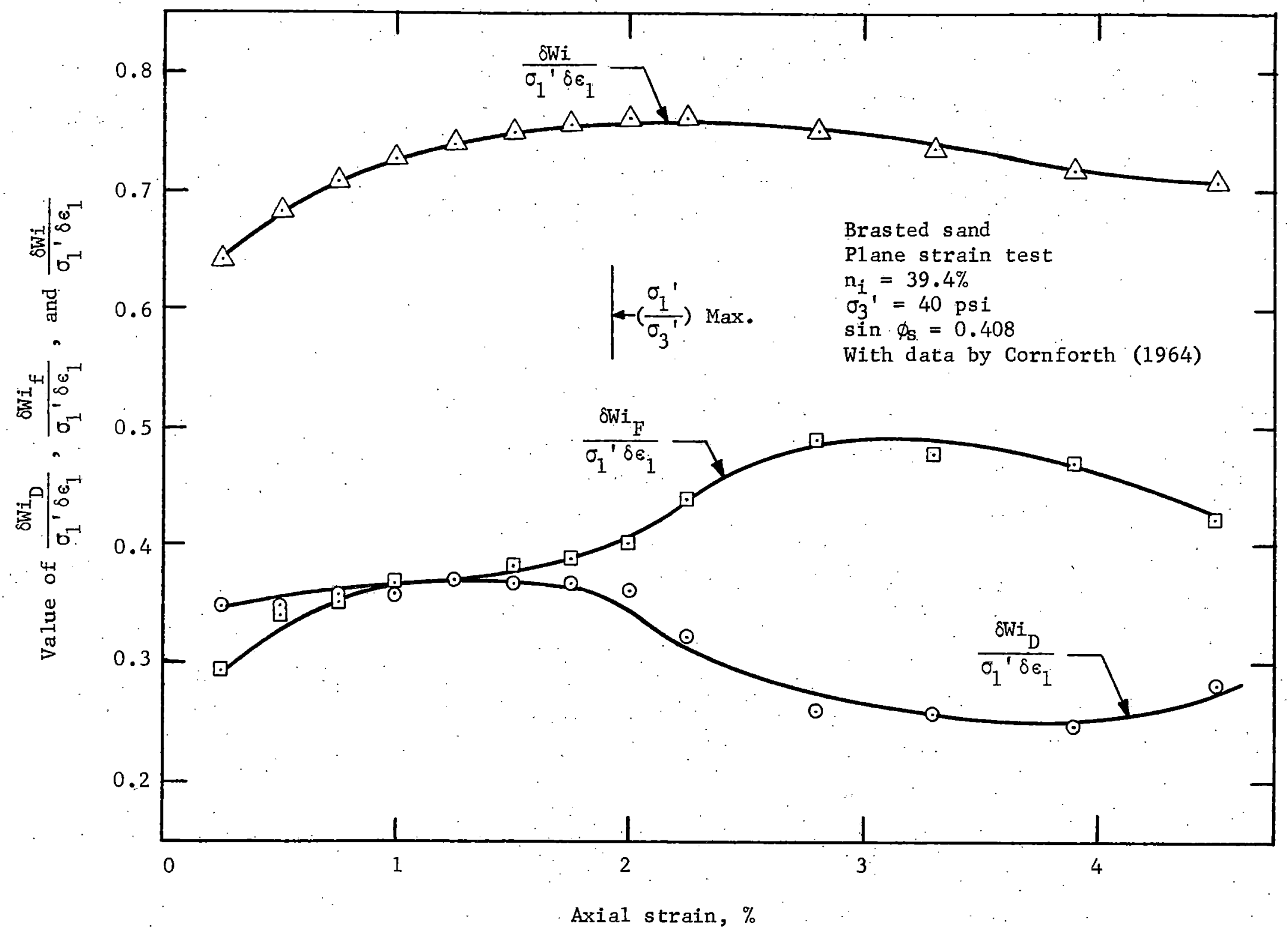

Fig. 16. Ratio of components of the rate of internal work to the rate of input work versus axial strain. 
loop made by the functions $\delta W i_{D} / \sigma_{1}^{\prime} \delta \epsilon_{1}$ and $\delta W i_{f} / \sigma_{1}^{\prime} \delta \epsilon_{1}$. The start of the loop, as seen in Fig. 16, is at the peak value. Then there is a rapid increase in the values of $\delta W i_{f} / \sigma_{1}^{\prime} \delta \epsilon_{1}$ with a corresponding decrease of the values of $\delta \mathrm{Wi}_{\mathrm{D}} / \sigma_{1}{ }^{\prime} \delta \epsilon_{1}$. The cause of this loop is the same as explained with reference to Fig. 14: namely, a breakdown of particle interference is required to allow an increase in the number of sliding contacts and, consequently, in the rate of internal work absorbed in friction.

Similar features are shown in Fig. 17 with data by Lambe (1951). Figure 17 allows one to follow the closing of the loop as the function $\delta W i / \sigma_{1}^{\prime} \delta \varepsilon_{1}$ starts to decrease in rate with respect to axial strain. One may hypothesize that the closing related to the appearance of a slip surface although the formation of such slip surface presumably may start when either the function $F$ or the function $\delta W i_{f} / \sigma_{1}{ }^{\prime} \delta \varepsilon_{1}$ has reached a maximum value. Since both occur after the peak, one may concur with Bishop and Green (1965) in concluding that the inhibition or lack of inhibition of preferential slip zones is a factor of no significance in determining the peak strength of granular materials.

Figure 18 represents the relationship between the function $\delta W_{D} /$ $\sigma_{3}{ }^{\prime} \delta \epsilon_{1}$ and porosity on the Brasted sand with data by Cornforth. (1964) and by Bishop and Eldin (1953). The effect of type of shear test, porosity, and level of confining pressure on the values of $\delta W_{\mathrm{D}} / \sigma_{3}{ }^{\prime} \delta \varepsilon_{1}$ may be studied in this figure.

It is obvious that increase in porosity decrease the values of $\delta W i_{D} / \sigma_{3} \cdot \delta \epsilon_{1}$ this may be expected from the standpoint that loose soil contract during shear to failure. The plane strain test performed with 


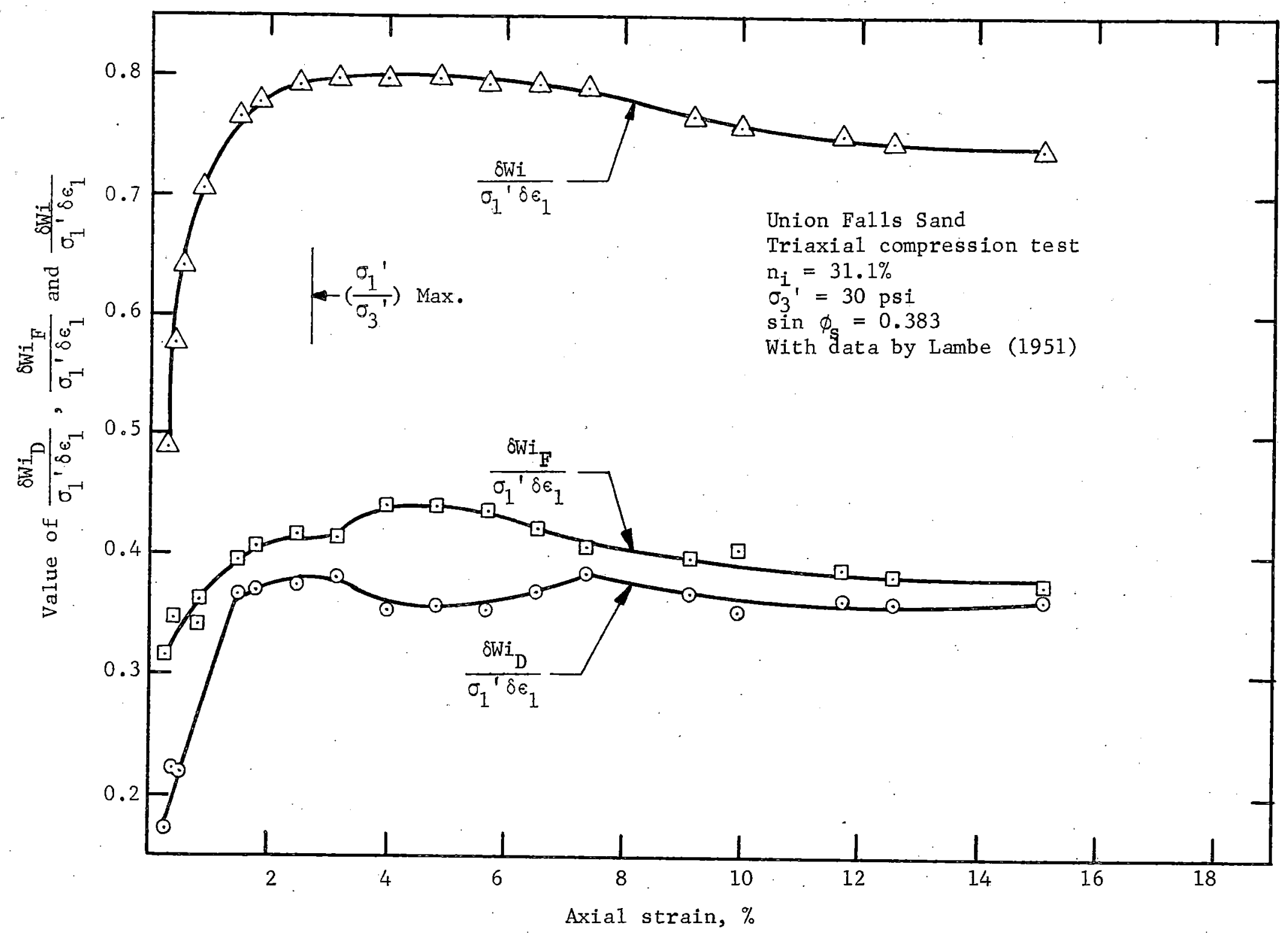

Fig. 17. Ratio of components of the rate of internal work to the rate of input work versus axial strain. 


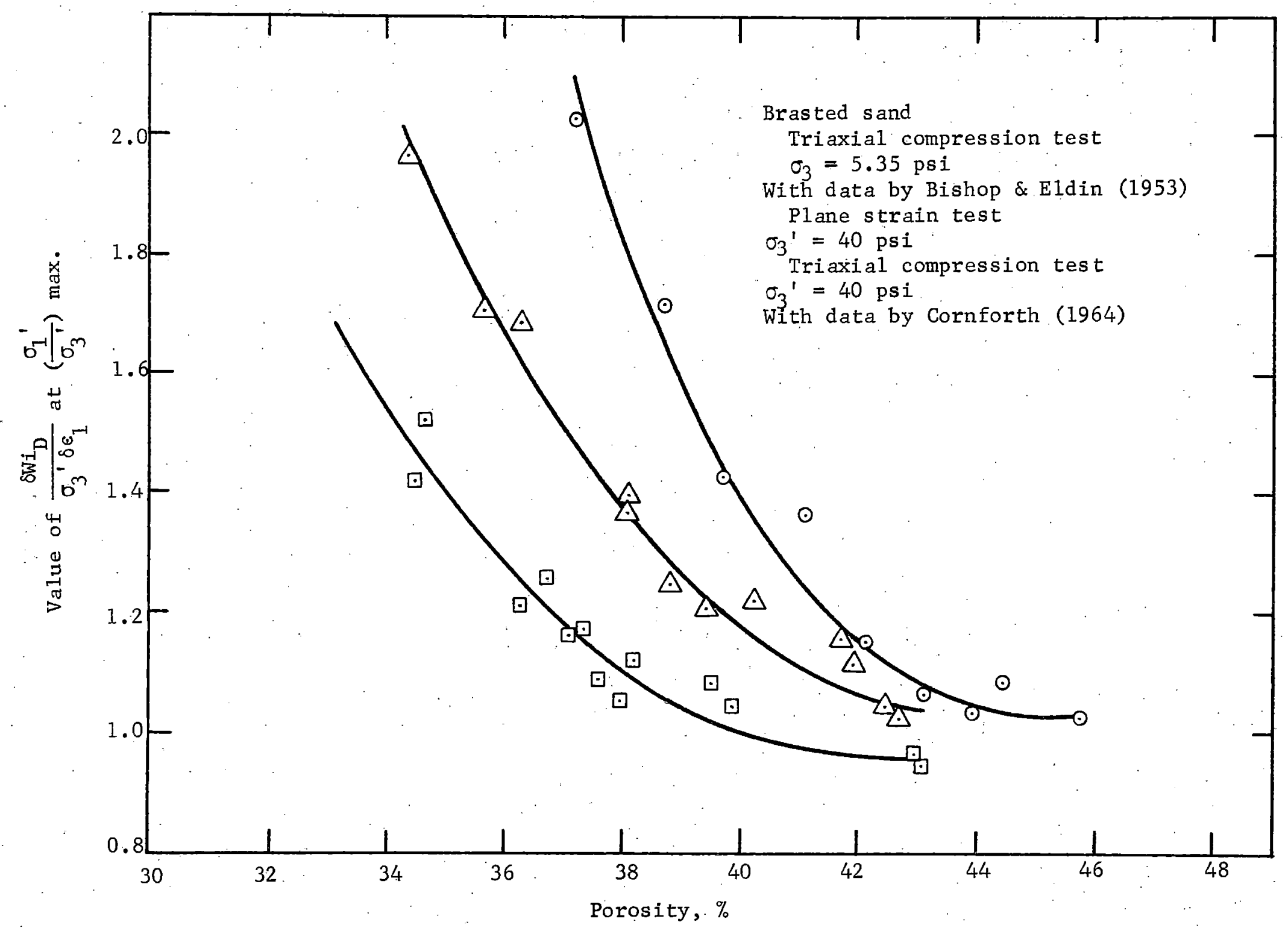

Fig. 18. Values of $\frac{\delta W i}{\sigma_{3}{ }^{\prime} \delta \varepsilon_{1}}$ at the peak $\frac{\sigma_{1}}{\sigma_{3}}$ in relation to porosities. 
a minor principal stress of $40 \mathrm{psi}$ showed higher values of $\delta \mathrm{Wi}_{\mathrm{D}} / \sigma_{3}{ }^{\prime} \delta \epsilon_{1}$ in the whole range of porosities than the triaxial compression test at same effective minor principal stress. This has been explained on the basis of the larger amount of particle interferences in the plane strain test due to the imposed strain conditions. The effect of confining pressure is to decrease the values of $\delta W_{\mathrm{D}} / \sigma_{3}{ }^{\prime} \delta \epsilon_{1}$ over the whole range of porosities tested. However, this relationship was changed when the ce11 pressure was increased to 60 psi, as shown in Fig. 19 . At lower porosities the values of $\delta \mathrm{Wi}_{\mathrm{D}} / \sigma_{3}{ }^{\prime} \delta \epsilon_{1}$ with the cell pressure of 60 psi are correspondingly higher than for a cell pressure of 40 psi, and the trend is reversed at higher porosities. The reason for the changing relationship is that increases in mean principal stresses increase the values of the parameter $\Omega$, as previously stated, and decrease the rate of volume change. However, this increased amount of interlocking in the samples at 60 psi does not bring a corresponding increase in the rate of internal work absorbed in friction, as shown in Fig. 21. Figure 20 indicates that larger confining pressures increase the amount of interna1 work absorbed in friction by the sample, but as the cell pressure is increased the rate of internal work absorbed in friction is decreased. This can be explained if increases in the values of the mean principal stresses, or octahedral stress, above a certain value may be inducing grain failure at contacts when the samples are at low porosities. This should increase interlocking, as shown below with reference to Fig. 22:

The relationship given by Eq. (9n) is presented in Fig. 22 for a sand tested at a confining pressure of 4,000 psi, from data by Bishop 


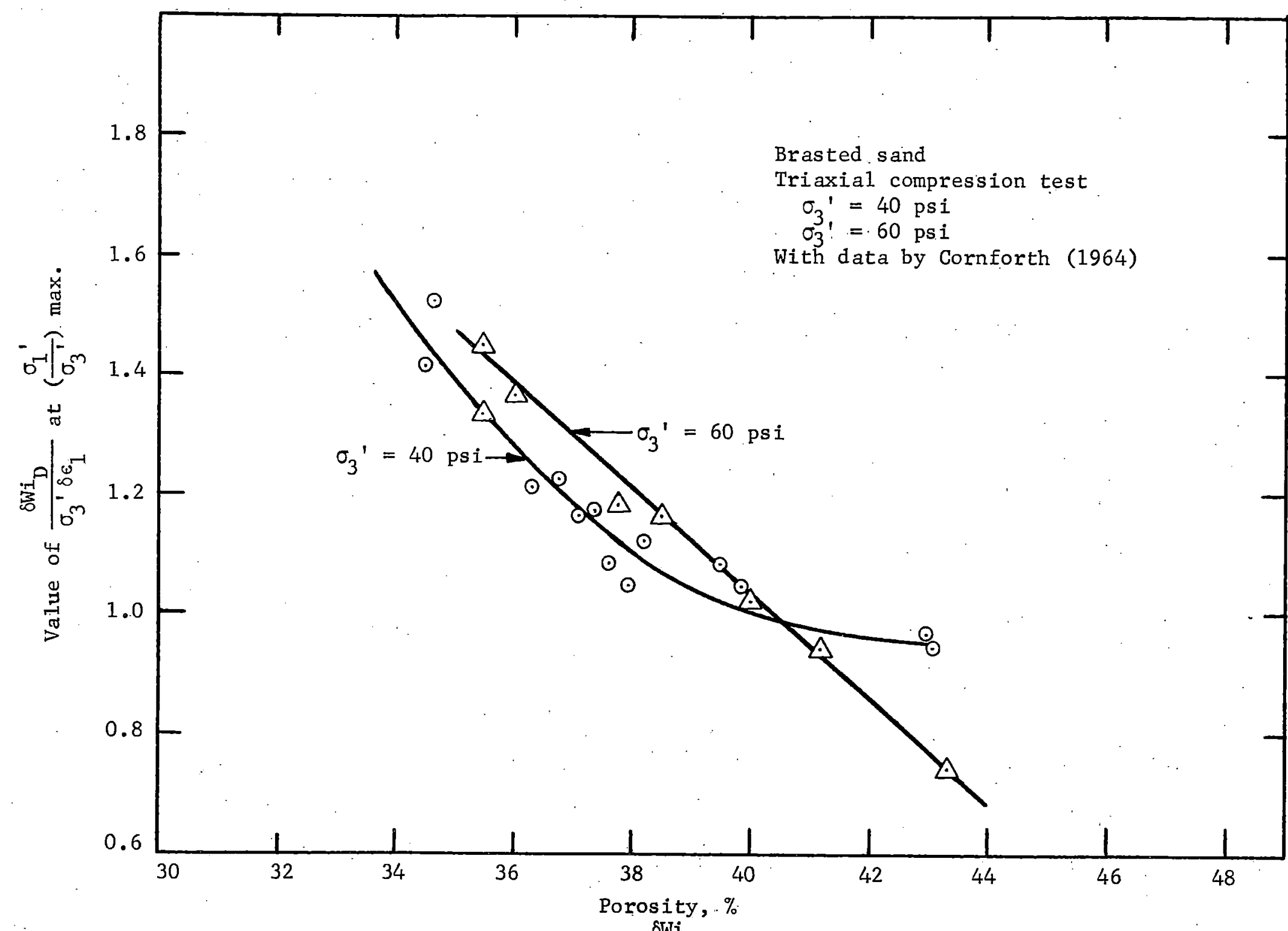

Fig. 19. Values of $\frac{\delta W i_{D}}{\sigma_{3}{ }^{\prime} \delta \epsilon_{1}}$ at the peak versus porosity. 

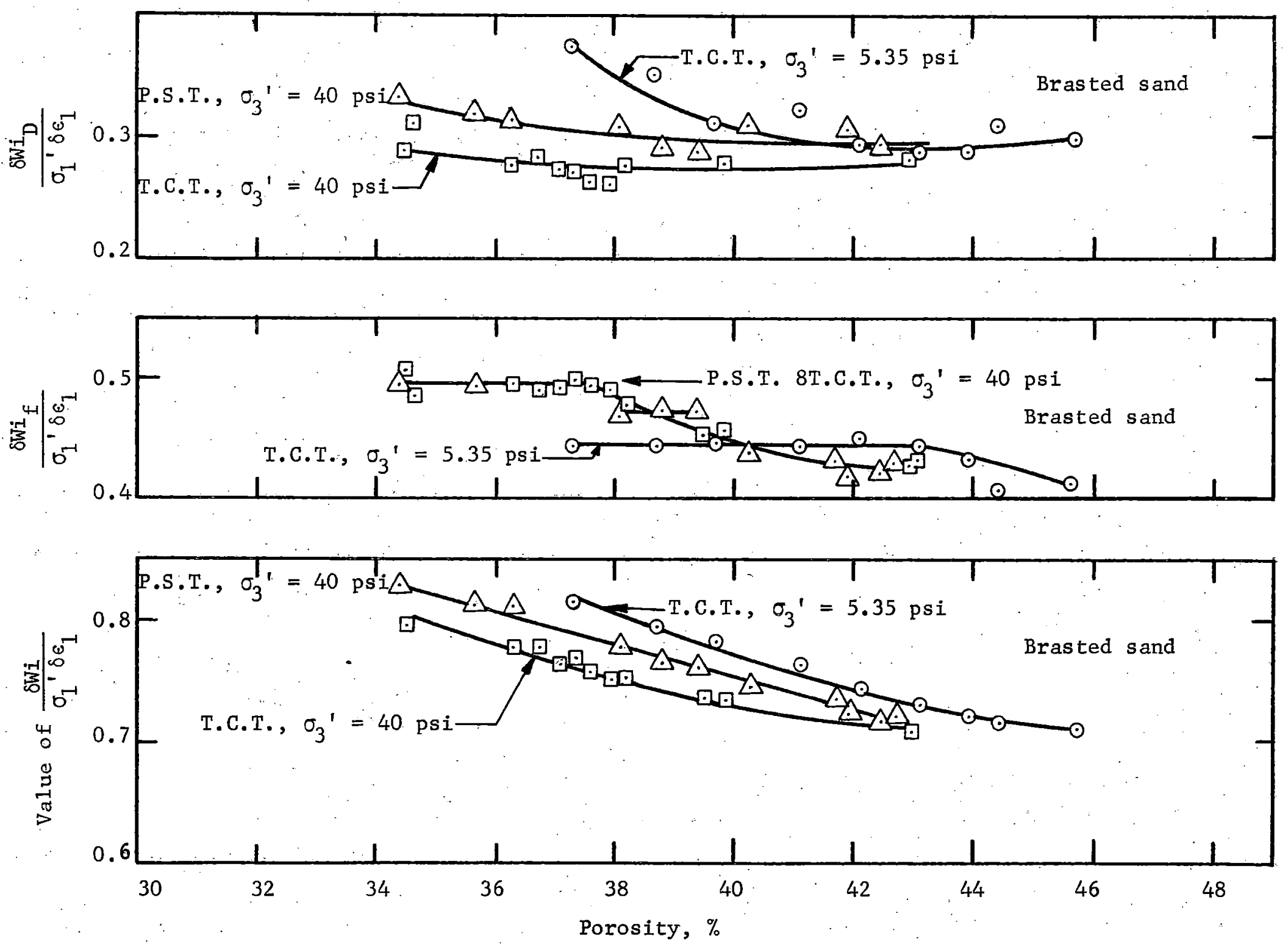

Fig. 20. Ratio of components of internal work to the rate of input work versus porosity. 

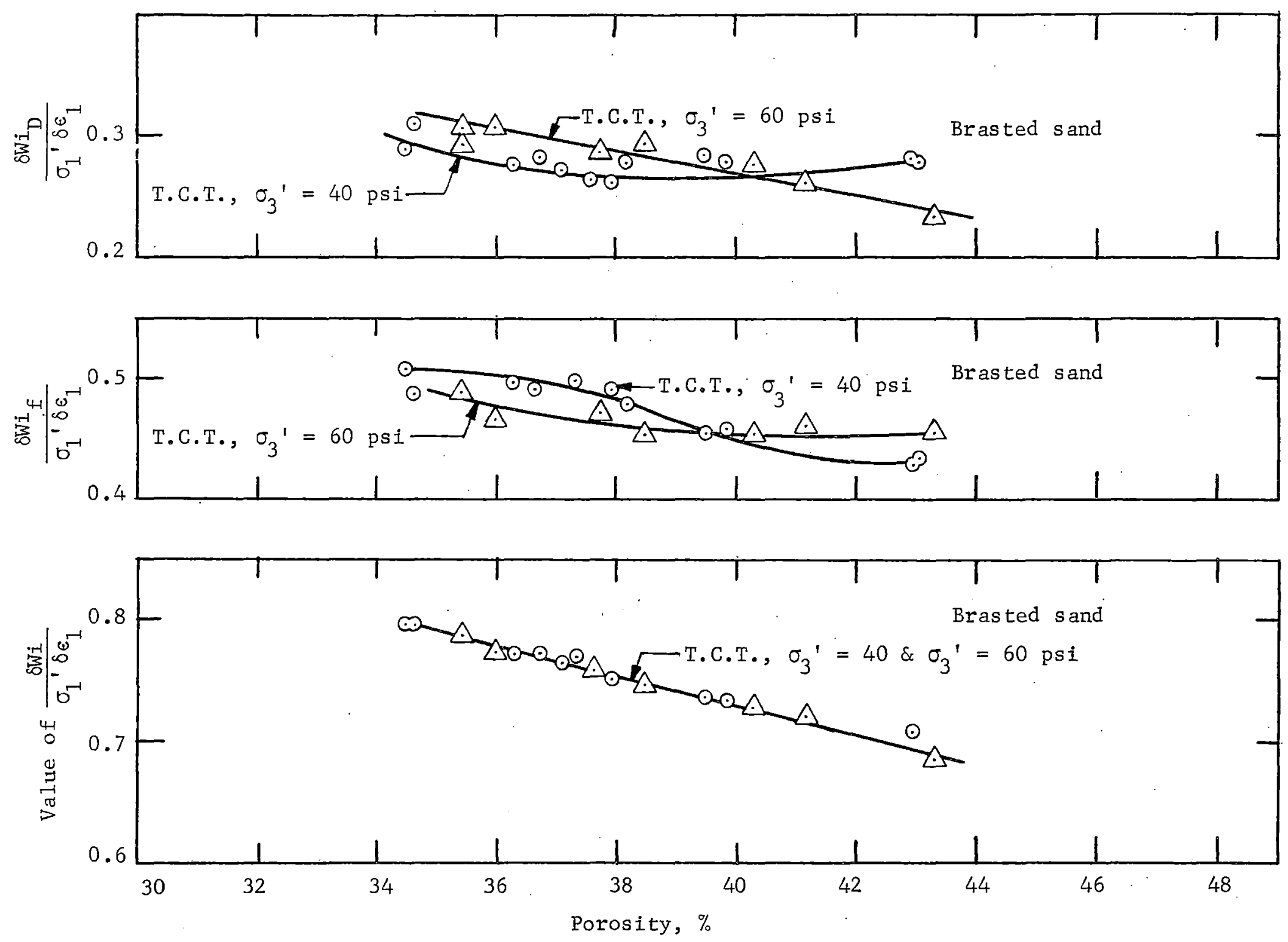

Fig. 21. Ratio of components of the internal work to the rate of input work versus porosity. 


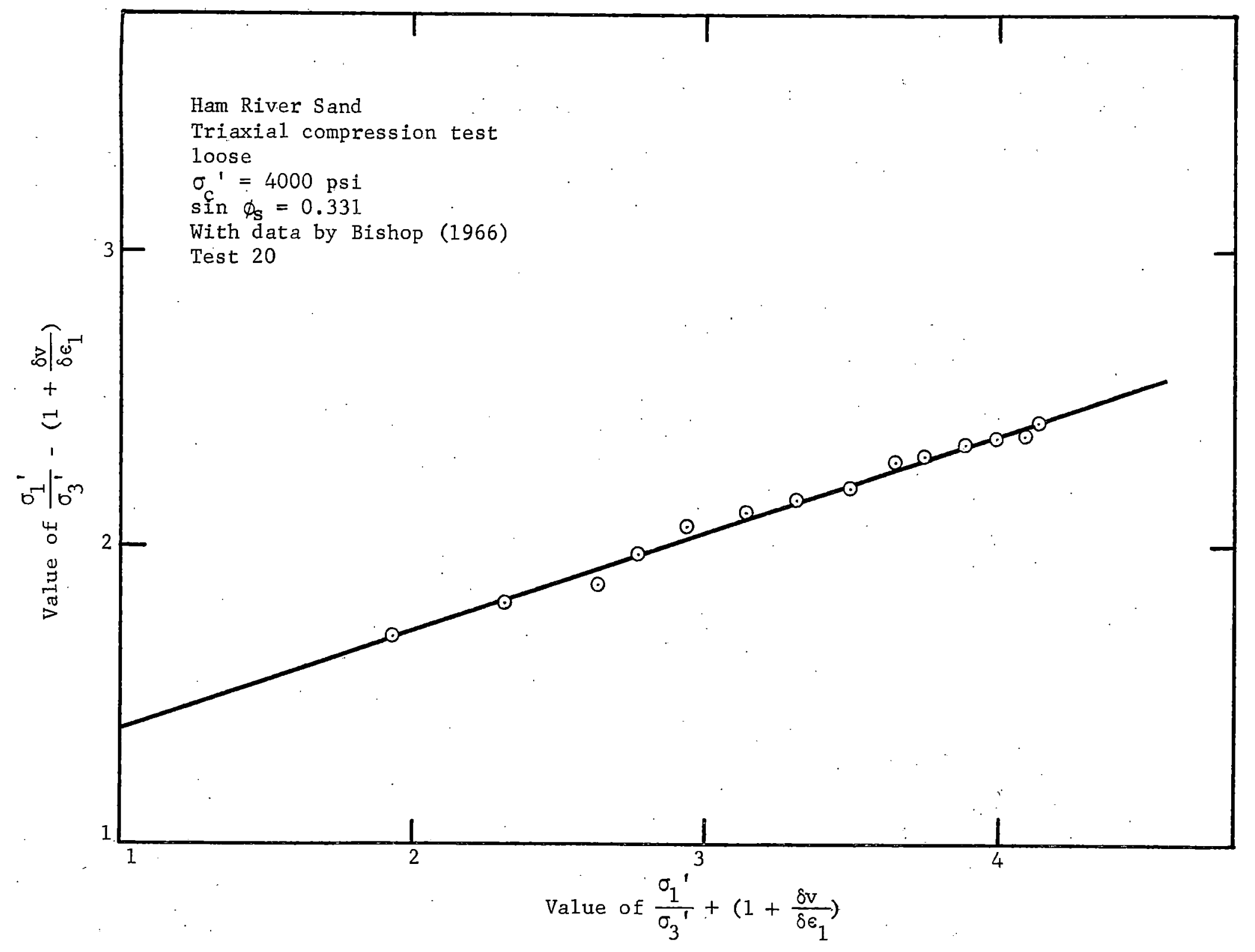

Fig. 22. Testing of Eq. (9n). 
(1966). The relationship is a straight line and the calculated values of angle of solid friction and the parameter $\Omega$ are 19.3 degrees and 1.057 , respectively. The values of the angle of solid friction and $\Omega$ for the same sand tested at a confining pressure or 40 psi with data by Bishop and Green (1965) and presented in Fig. 9 are 24.1 degrees and 0.395 , respectively. Thus an extremely high confining pressure produced a decrease in the angle of solid friction and an increase in the value of $\Omega$. Bishop (1966) showed that grain failure occurred within the sample at this high confining pressure, and showed that the gradation of the medium to fine sand was changed to a gradation corresponding to a silty sand. The calculated values of the angle of solid friction and of the parameter $\Omega$ at the high confining pressure do not represent true values, because the development of the theory assumes no grain failure at the contacts. That is, the rate of work internally absorbed by the sample in fracturing individual grains must be added to the calculated rates of internal work absorbed in friction and dilatancy. However, the extrapolation of the theory beyond its limitations serves the purpose of explaining the mechanism of the changing relationship from increases in cell pressure within a given range.

It may now be shown that if the mean effective principal stress (or effective octahedral stress) is kept constant, the value of $\Omega$ must decrease. This is shown in Fig. 23, which represents the relationship given by Eq. (9n) for a triaxial compression test on feldspar where the effective octahedral stress was kept constant and equal to 30 psi, data are by Lee (1966). The calculated value of $\Omega$ is 0.04 , which may 


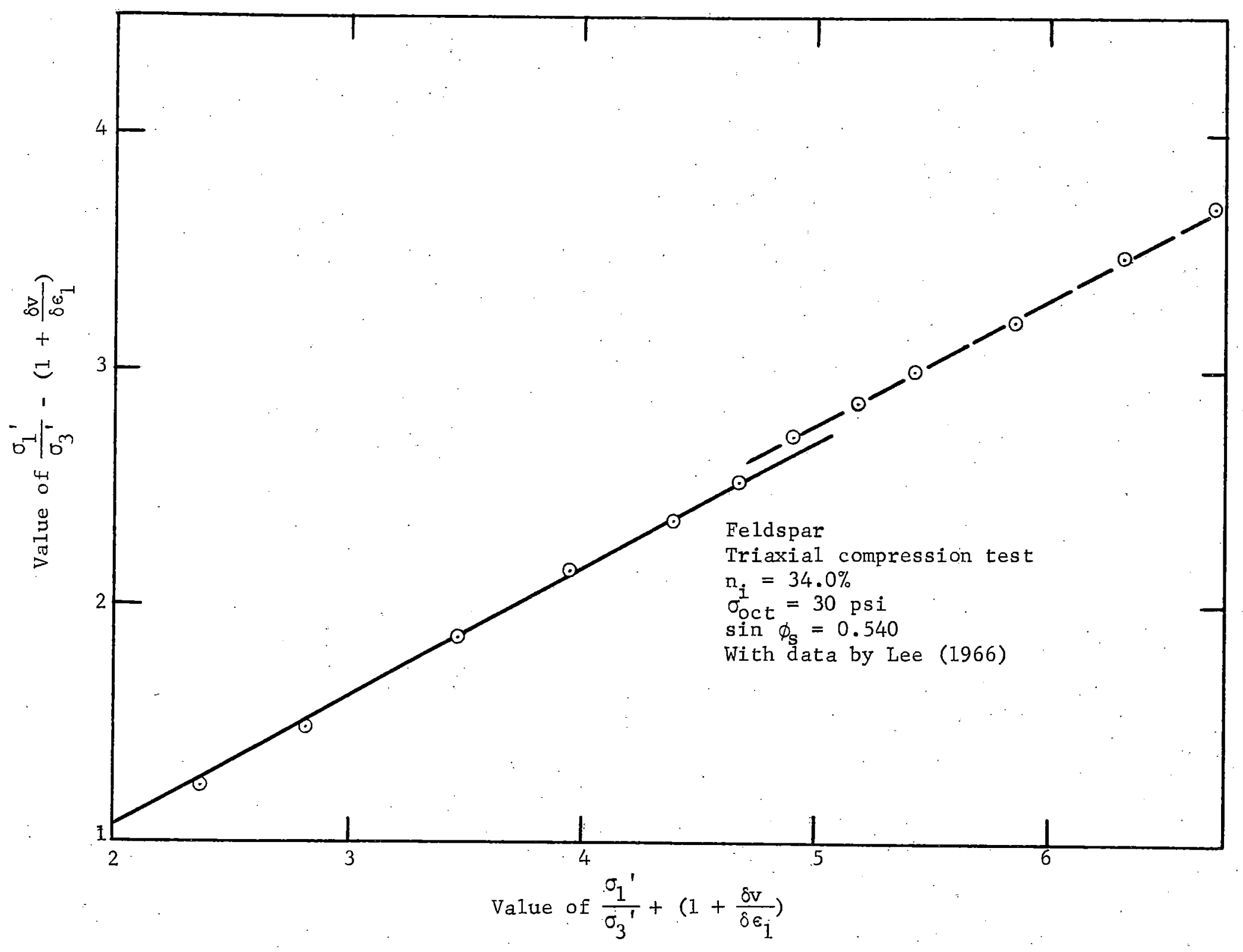

Fig. 23. Testing of Eq. (9n). 
compared with a value of $\Omega$ of 0.390 for a test on the same material at a constant cell pressure equal to $30 \mathrm{psi}$ also with data by Lee (1966)。

The first two point values corresponding to very. small deformations are not shown in Fig. 23, and fell below the solid line in Fig. 23. The calculated values of the parameter $\Omega$ for these points gave high negative values, indicating that during the initial stages of deformation the preferential mechanism of distortion is not by sliding but rather by compression, which would explain the negative values of $\Omega_{0}$ 


\section{SUMMARY}

1. A theory was developed to allow the separate determination of effects of the interparticle friction and of geometric constraints among the particles on the shearing resistance and deformational behavior of granular materials.

2. According to the theory the effect of interparticle friction may be measured by the angle of solid friction. The calculated angle of solid friction was found to be independent of the type of shear test, stress history, porosity, and the level of the confining pressure in the ranges commonly used in soil shear testing. The angle of solid friction depends only on the nature of the particle surfaces.

3. The effect of the geometric constraints is measured by the parameter $\Omega$, which $\Omega$ was found to depend on the gradation of the granular material, initial porosity, type of shear test, and the level of the confining_pressure.

4. The parameter $\Omega$ allowed the calculation of components of the rate of internal work absorbed by the sample. The calculation of these components and their changes throughout deformation allowed the qualitative examination of the deformational behavior of granular materials and justified the postulated mechanism of deformation. 


\section{PART II. SHEAR STRENGTH OF CRUSHED LIMESTONES MATERIALS}

Three crushed stones were selected as representative of Iowa State Highway Commission-approved crushed stone for rolled-stone bases. One is a weathered, moderately hard limestone of the Pennsylvanian system, obtained from near Bedford, in Taylor County, Iowa, and hereafter referred to as the Bedford sample. The second is a hard, concrete quality limestone of the Mississipian system, obtained from near Gilmore City, Humboldt County, Iowa, and hereafter referred to as the Gilmore sample; and the third is a hard dolomite of the Devonian system, obtained near Garner, Hancock County, Iowa and hereafter referred to as the Garner sample.

X-ray diffraction analysis (Hoover, 1965) of powdered representative samples showed calcite as the predominant mineral in the three stones, but there was a considerable difference in calcite-dolomite ratio, ranging from 25 in the Bedford stone to 1.16 in the Garner. $\mathrm{X}$-ray tests on $\mathrm{HCl}$-insoluble residues showed no montmorillonite in any of the samples, a small amount of vermiculite or chlorite in the Garner, a predominance of illite in the Bedford and Garner samples, plus kaolinite and quartz. Kaolinite in the Bedford stone was poorly crystalline and quartz was almost nonexistent in the Gilmore sample. The percent of insoluble residues were $10.9,6.70$, and 1.66 in the Bedford, Garner, and Gilmore, respectively. Cation exchange capacities and $\mathrm{pH}^{\prime} \mathrm{s}$ of the whole samples were closely comparable. Engineering properties of the three crushed stones are shown in Table 2. The Bedford sample contains more gravel, less sand, and 
more clay size particles, and has a measurable plasticity. The optimum moisture content for compaction is higher and the compacted density is lower than for the Garner and Gilmore samples.

Table 2. Representative engineering properties of crushed stone materials.

\begin{tabular}{|c|c|c|c|}
\hline & Bedford & Garner & Gi1more \\
\hline \multicolumn{4}{|l|}{ Textura1 Composition, \% } \\
\hline Gravel $(2.00 \mathrm{~mm})$ & 73.2 & 61.6 & 66.8 \\
\hline Sand $(2.00-0.074 \mathrm{~mm})$ & 12.9 & 26.0 & 23.3 \\
\hline Silt $(0.074-0.005 \mathrm{~mm})$ & 8.4 & 10.2 & 5.9 \\
\hline Clay $(0.005 \mathrm{~mm})$ & 5.5 & 2.2 & 4.0 \\
\hline Colloids $(0.001 \mathrm{~mm})$ & 1.7 & 1.4 & 0.9 \\
\hline \multicolumn{4}{|l|}{ Atterberg Limits, \% } \\
\hline Liquid limit & 20.0 & Non- & Non- \\
\hline Plastic limit & 18.0 & Plastic & Plastic \\
\hline P1asticity index & 2.0 & & \\
\hline \multicolumn{4}{|c|}{ Standard AASHO-ASTM Density: } \\
\hline \multicolumn{4}{|c|}{ Optimum moisture content, } \\
\hline$\%$ dry soil weight & 10.8 & 7.6 & 9.3 \\
\hline Dry Density, pcf. & 128.0 & 140.5 & 130.8 \\
\hline \multicolumn{4}{|c|}{ Modified AASHO-ASTM Density: } \\
\hline \multicolumn{4}{|c|}{ Optimum moisture content } \\
\hline$\%$ dry soil weight & 8.0 & 5.4 & 5.7 \\
\hline Dry density, pcf. & 133.5 & 147.6 & 140.8 \\
\hline \multicolumn{4}{|l|}{ Specific Gravity of Minus } \\
\hline No. 10 sieve fraction & 2.73 & 2.83 & 2.76 \\
\hline \multicolumn{2}{|l|}{ Textural Classification } & \multicolumn{2}{|c|}{ Grave11y Sandy Loam } \\
\hline AASHO Classification & $A-1-b$ & $A-1-a$ & $A-1-a$ \\
\hline
\end{tabular}




\section{METHODS OF INVESTIGATION}

For the laboratory measurement of shear strength under controlled conditions of drainage and deformation, the engineer is largely dependent on the triaxial test, or the cylindrical compression test. The test may, however, be performed in various ways.

The type of test selected for this investigation was the isotropically consolidated-undrained triaxial test or CIU test. The term isotropically consolidated is a misnomer but is widely used in the soil mechanics literature. Isotropically consolidated means that the soil is consolidated under an equal all-around pressure.

\section{TRIAXIAL SPECIMEN PREPARATION}

Previous studies have indicated that granular materials are more suitably compacted using vibratory methods. This method was chosen for the compaction of the triaxial specimens ( 4 in. by 8 in. cylinders) to the standard Proctor density as determined by AASHO/ASTM procedures.

A syntron, Mode1 V-60, electromagnetic vibrator table with a constant frequency of 3600 vibrations per minute was used. The ampli-. tude could be varied with a rheostat graduated from 0 to 100 .

Hoover (1965) found that this size triaxial specimen could be compacted to Standard Proctor density with little or no particle degradation and segregation by using the following combination of factors:

1. Rheostat dial setting of 90 , for an amplitude of 0.368 in.

2. Period of vibration of two minutes.

3. Surcharge weight of 35 pounds. 
No moisture-density relationship was determined for the vibratory compaction; the moisture content being obtained from standard Proctor compaction. The dry density of the crushed stones was the controlling factor in the preparation of the triaxial specimens. However, vibratory compaction of the Garner limestone yielded a dry density higher than standard Proctor.

Preparation of the triaxial shear specimens began by air-drying sufficient crushed stones for a 4 in. by 8 in. specimen. Distilled water was added to obtain the optimum moisture content. All mixing was accomplished by hand to prevent degradation and segregation of the material. The mix was added to the mold in three equal layers, each layer being rodded 25 times with a 5/8 in.-diam tapered-point steel rod. The surcharge weight of 35 pounds was placed on top of the specimen and compaction was achieved in accordance with the previously mentioned specifications.

After removal from the vibrator table, each specimen was extruded from the mold by hydraulic jacking. The specimen was wrapped in a double layer of Saran Wrap and aluminum foil, weighed, and placed in a curing room, at near $75^{\circ} \mathrm{F}$ and 100 percent relative humidity until testing time.

\section{TRIAXIAL APPARATUS}

The triaxial apparatus used in this investigation was designed in the Engineering Research Institute Soi1 Research Laboratory and built by the Engineering Research Institute shop. The unit consists of two bays capable of testing two specimens simultaneously under different lateral pressure and drainage conditions. 
Rate of strain can be varied between 0.0001 and near 0.1 in. per minute. The set rate is constant within $1 / 2$ of 1 percent under a11 loads, as produced through a combination of a Dynamatic Adjust-oSpeed Motor controlled by a Dynamatic Silicon Controlled Rectifier, Turner Two-speed Transmission, and Link Belt Worm Gear Speed Reducer. A maximum axial load of 11,000 pounds can be transferred to a specimen through a calibrated proving ring. The vertical deflection of the specimen is measured with a dial gage extensometer.

Lateral pressures can be applied to a specimen within a plexiglass cel1 by an air over liquid system or by air pressure only. This pressure can be varied between 0 and $100 \mathrm{psi}$ and is held within \pm 0.3 psi throughout a test by means of a diaphragm regulator.

Pore water pressures are measured at the base of the specimen through a 4 in.-diam porous stone by a Karo1-Warner Mode1 53-PP pore pressure device which operates on the null-balance principle, measuring both positive ( 0 to 100 psi) and negative pore pressures ( 0 to 15 psi). Specimen volume changes can be obtained, when water is used in the cell, to a precision of $0.01 \mathrm{cu}$ in. This is determined by maintaining a constant water level within the cell and measuring the amount of water that flows from or into an adjustable graduated tube that is under pressure equal to the applied cell pressure.

\section{ISOTROPICALLY CONSOLIDATED - UNDRAINED TRIAXIAL TES T}

Every specimen obtained from the curing room was weighed, measured, and placed in the triaxial cell. Each specimen was sealed in a rubber 
membrane of uniform 0.025 in.-wal1-thickness, and had a saturated 1/2-in.-thick corrundum porous stone on the top and bottom. The ce11 was filled with water to a fixed height, and all-around pressure was applied and drainage permitted through the base of the specimen during the consolidation phase. Volume change measurements were made at time intervals of $2,4,9,16,25,36$, and 49 minutes. A time of 49 minutes was found adequate for consolidation of all specimens.

After consolidation was complete, the specimen was sheared with the drainage valve closed and the pore pressure device incorporated into the system. The axial load was applied at a constant axial strain rate of 0.01 in. per minute. Volume change, pore pressure, and axial load (from proving ring deflection) were recorded at $0.010 \mathrm{in}$. interva1s up to $0.250 \mathrm{in}$. vertical deflection, and then every 0.025 in. up to 1 in. or more of vertical deflection depending on the deformation characteristics of the specimen. The specimen was removed at the end of the test, weighed, and three portions from the top, middle and bottom were taken for moisture content determinations.

Composite specimens of each stone were tested at lateral pressures of $10,20,30,40,60$, and 80 psi.

Selected specimens also were tested with slight variations from the above procedure. Bedford limestone specimens were tested under a "repeated" loading condition whereby the specimen was first loaded up to 0.075 in. of total axial deflection and then unloaded. After a waiting period to allow for equilibration, the specimen was loaded again to an additional 0.075 in. of axial deflection and unloaded. After equilibrium was again reached, the specimen was loaded as previously indicated. 
Some specimens of the Gilmore limestone were loaded under "repeated" loading, but with a different procedure. The specimen was axially loaded up to the maximum effective principal stress ratio and then unloaded. After equilibrium was achieved, the specimen was loaded as previously indicated.

Calculation and reduction of the triaxial test data were accomplished by using an IBM 360 Computer program and a 1627 L-Comp plotter through the Iowa State University Computer Center. These data provided a print-out of all relations and a plot of the effective stress ratio, volume change and pore pressure versus percent strain. These data wers then used for the analyses.

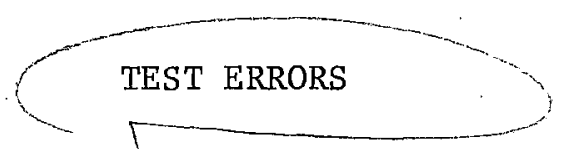

Nonuniformity of stress and deformation are by far the most important sources of error since if deformation is localized, the usual overall measurements are misleading. This can also lead to errors in the calculated value of the deviator stress, as indicated in the following paragraph. Nonuniformity may be introduced initially in the preparation of the sample, but its main cause is friction at the end plattens, which will modify the values of volume change and the slope of the stress-strain curve. Nevertheless the maximum values of the stress ratio should agree regardless of testing with friction or frictionless ends (Bishop and Green, 1965). Friction at the end plattens will produce two elastic cones at the ends of the sample, with the result that the middle zone will bulge more than the end zones and the deformed sample will take on a barrel shape. 
Errors in the calculated values of deviator stress are mainly caused by piston friction and also by the wrong area correction, which is related to the above-cited nonuniformity of deformation. The errors involved become unacceptable for axial strains larger than 10 percent of the initial height of the sample:

Axial strain is usually calculated from the axial displacements of the piston measured relative to the initial position of the piston. Errors in the measured axial strain are thus caused by friction in the piston and bedding errors related to the initial positioning of the piston.

The method used for calculating the volumetric strain is by measuring the volume of water entering or leaving the cell. The main sources of error are nonuniform deformation, evaporation, leakage and membrane penetration. Evaporation and leakage are minor errors and can be totally eliminated in tests of short duration. Errors due to membrane penetration are related to the shape and size of the particle and to nonuniform deformation.

Al1 these errors can and should be minimized by modification in the design of the cell and measuring instrumentation. However, most of the above-mentioned errors were present in some amount in the testing program, and must be kept in mind in evaluating the data. 


\section{DISCUSSION OF TEST RESULTS}

$$
\text { Page } 29
$$

Equation (9n) was applied to selected results from the numerous triaxial tests performed on crushed limestones. The selection was based on variables which may have an influence on the behavior of the crushed limestones, such as initial void ratio, level of confining pressure, stress history, and change in the gradation of the sample. These test results are shown in Figs. 24 to 30 .

Linear relations were found over a wide deformation range regardless of the variables being investigated. Two features common to all of Figs. 24 to 30 are first the existence of more than one linear relation, and second, deviation from a linear relation during the initial stages of deformation (less than one percent of the axial strain).

The existence of more than one linear relation was previous $1 y$ explained to be due to the use of "fixed" ends, which introduce non-uniformity of strains within the sample.

Reasons for deviations from a linear relation during the initial stages of deformation will be advanced when references are made to Figs. 32 to 36 .

\section{Solid Friction and Dilatancy}

Table 3 is summary of selected test results. The calculated value of the solid friction angle of the limestones was 34.2 degrees regardless of initial void ratio, stress history, gradation and packing, and level of confining pressure. 


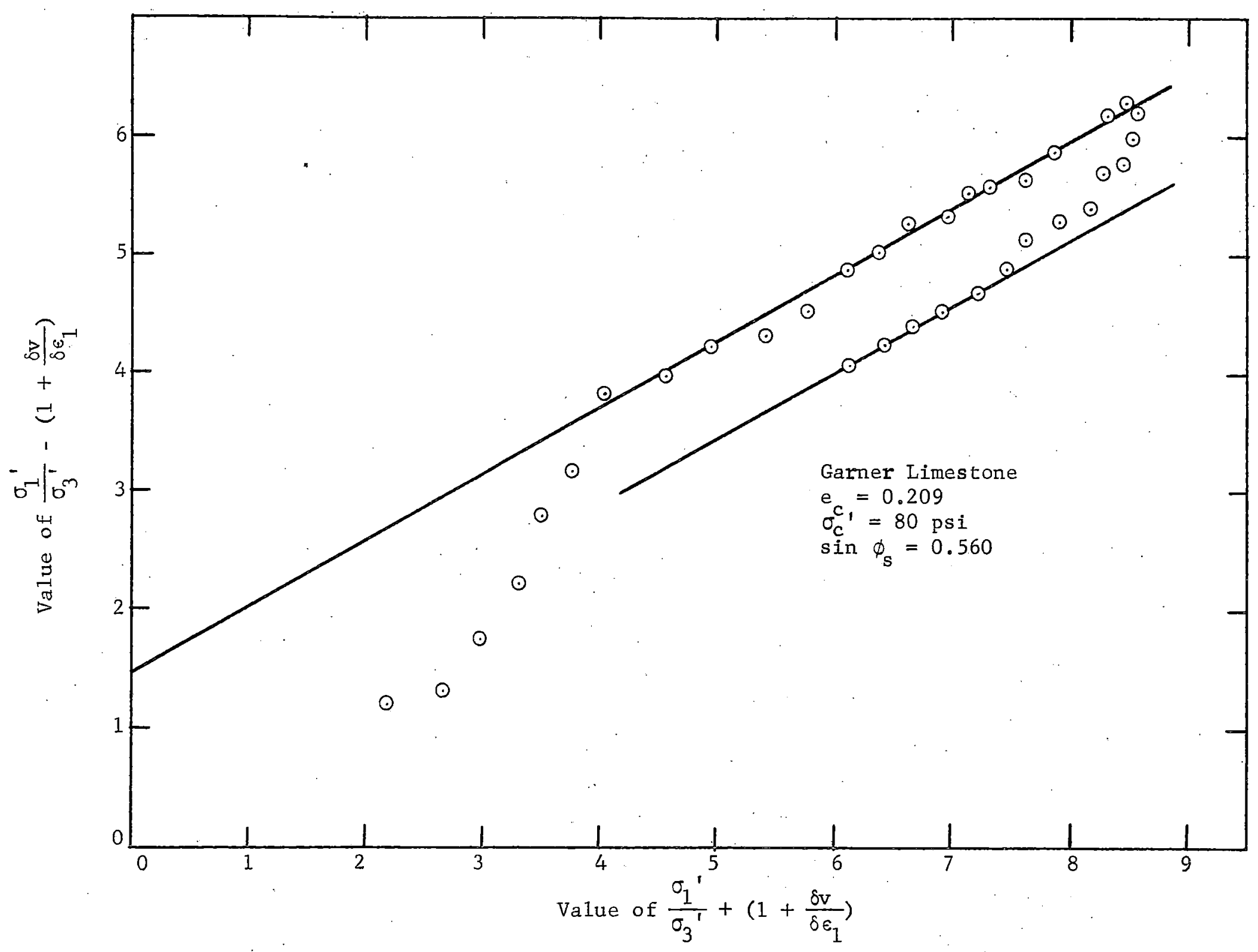

Fig. 24. Application of Eq. (9n) to tests on crushed limestones. 


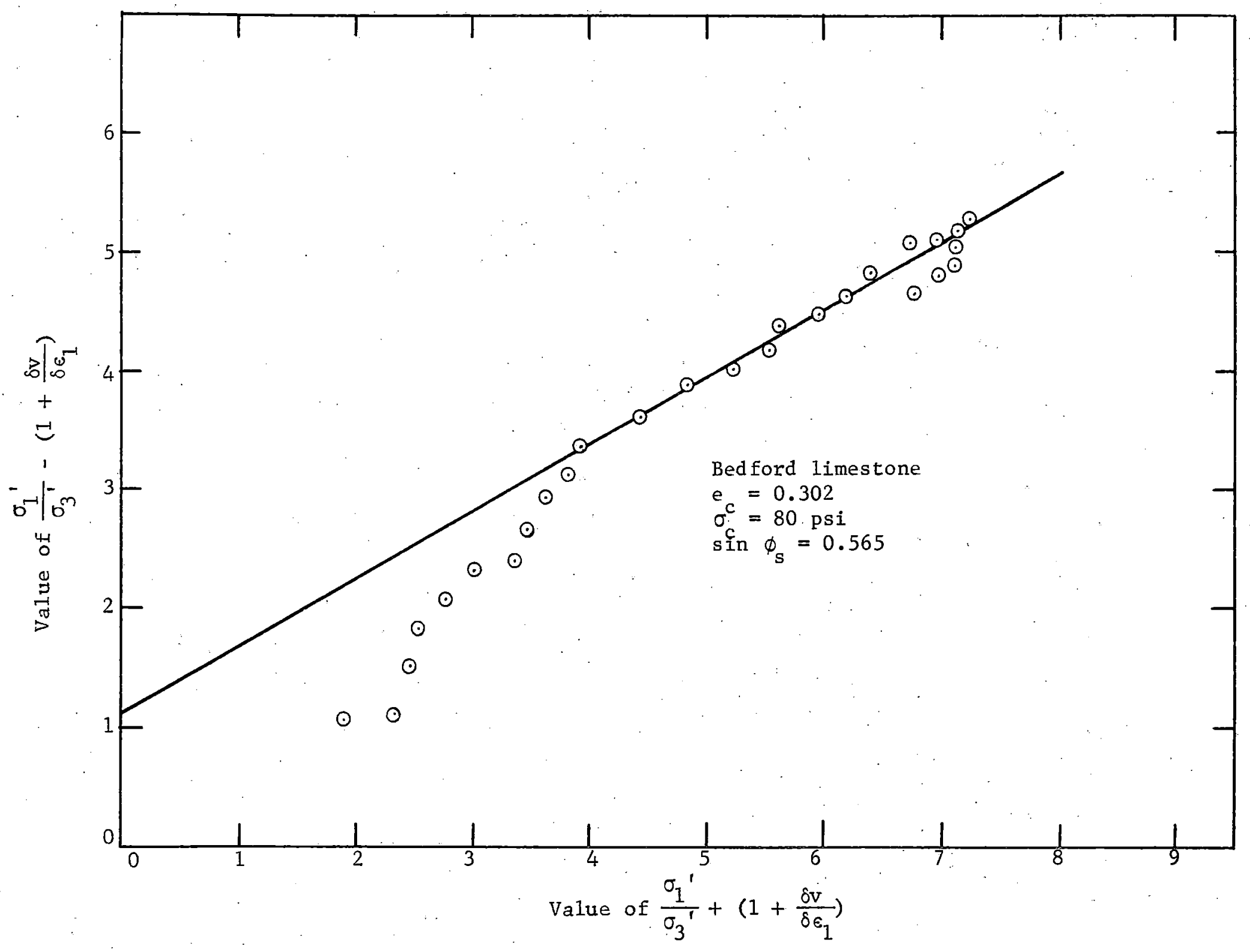

Fig. 25. Application of Eq. (9n) to tests on crushed limestones. 


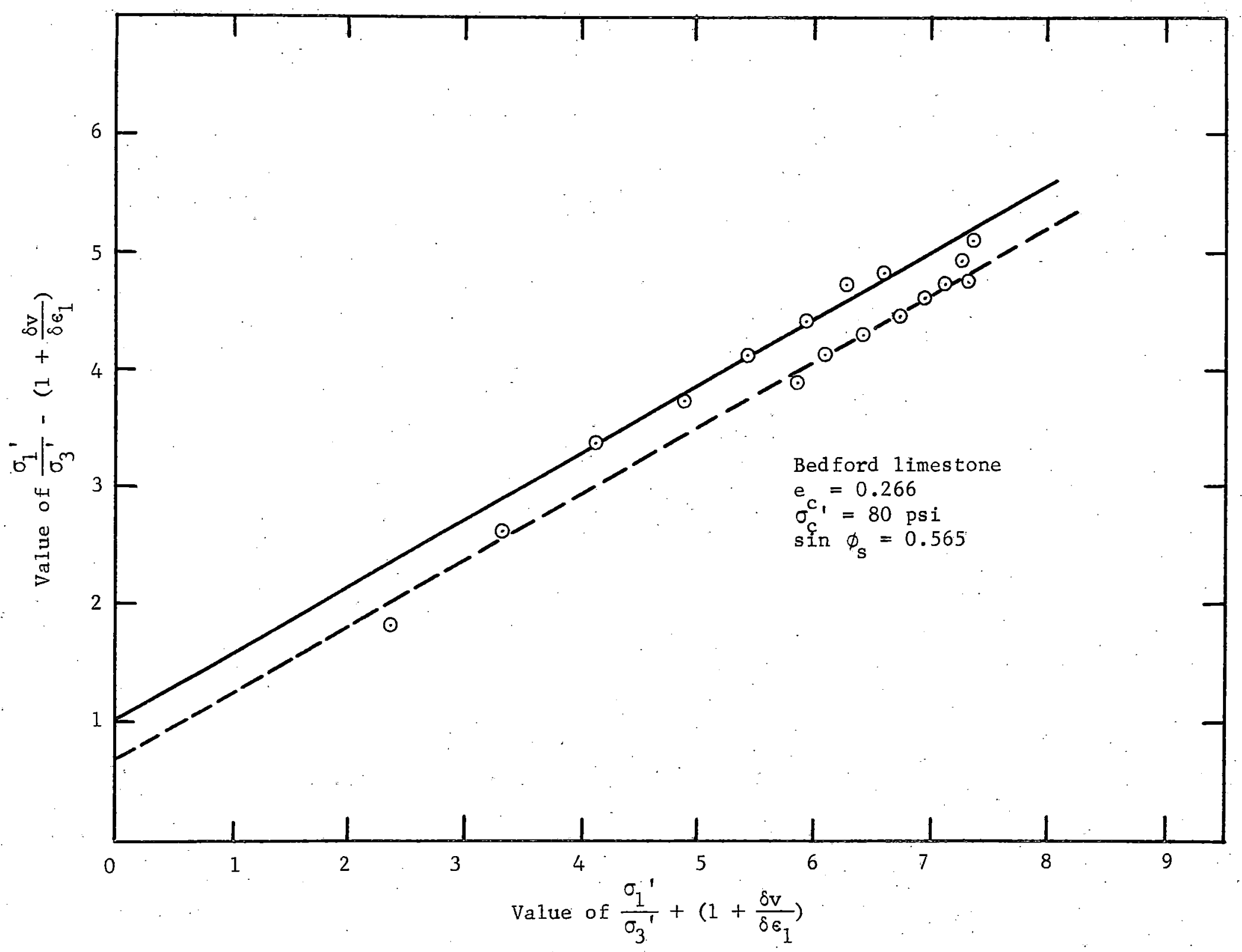

Fig. 26. Application of Eq. $(9 n)$ to tests on crushed limestones. 


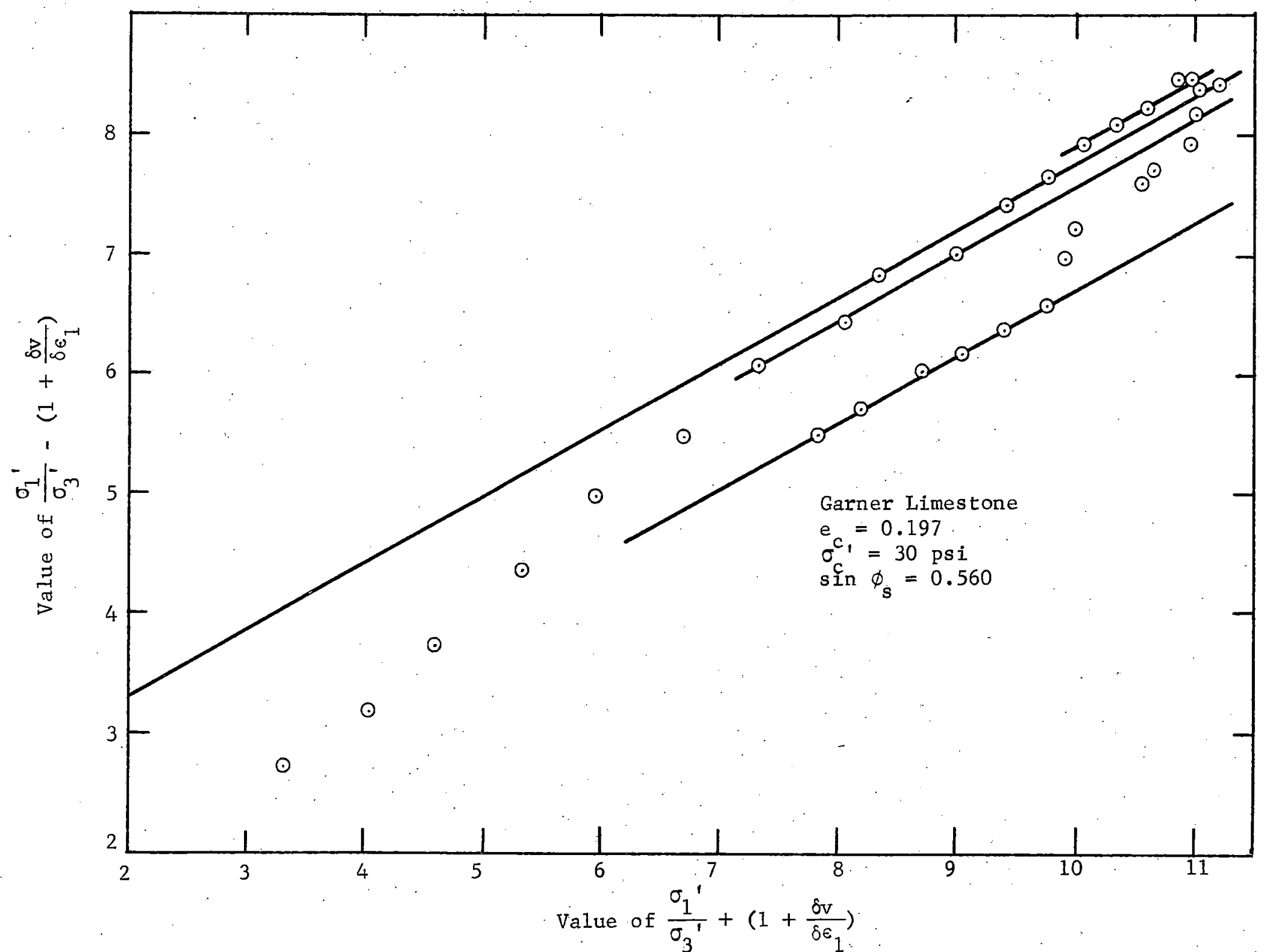

Fig. 27. Application of Eq: (9n) to tests on crushed limestones. 


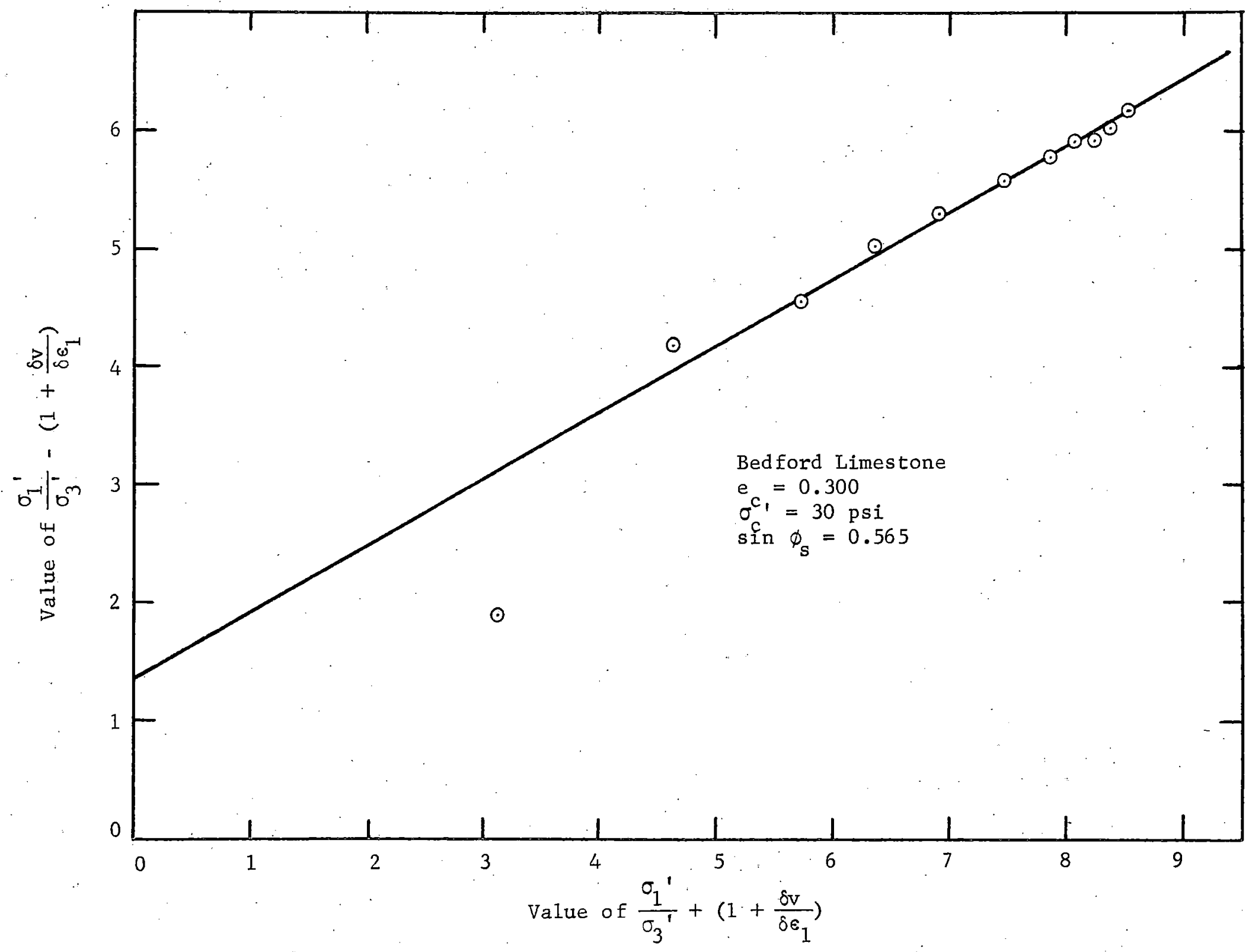

Fig. 28. Application of Eq. (9n) to tests on crushed limestones. 


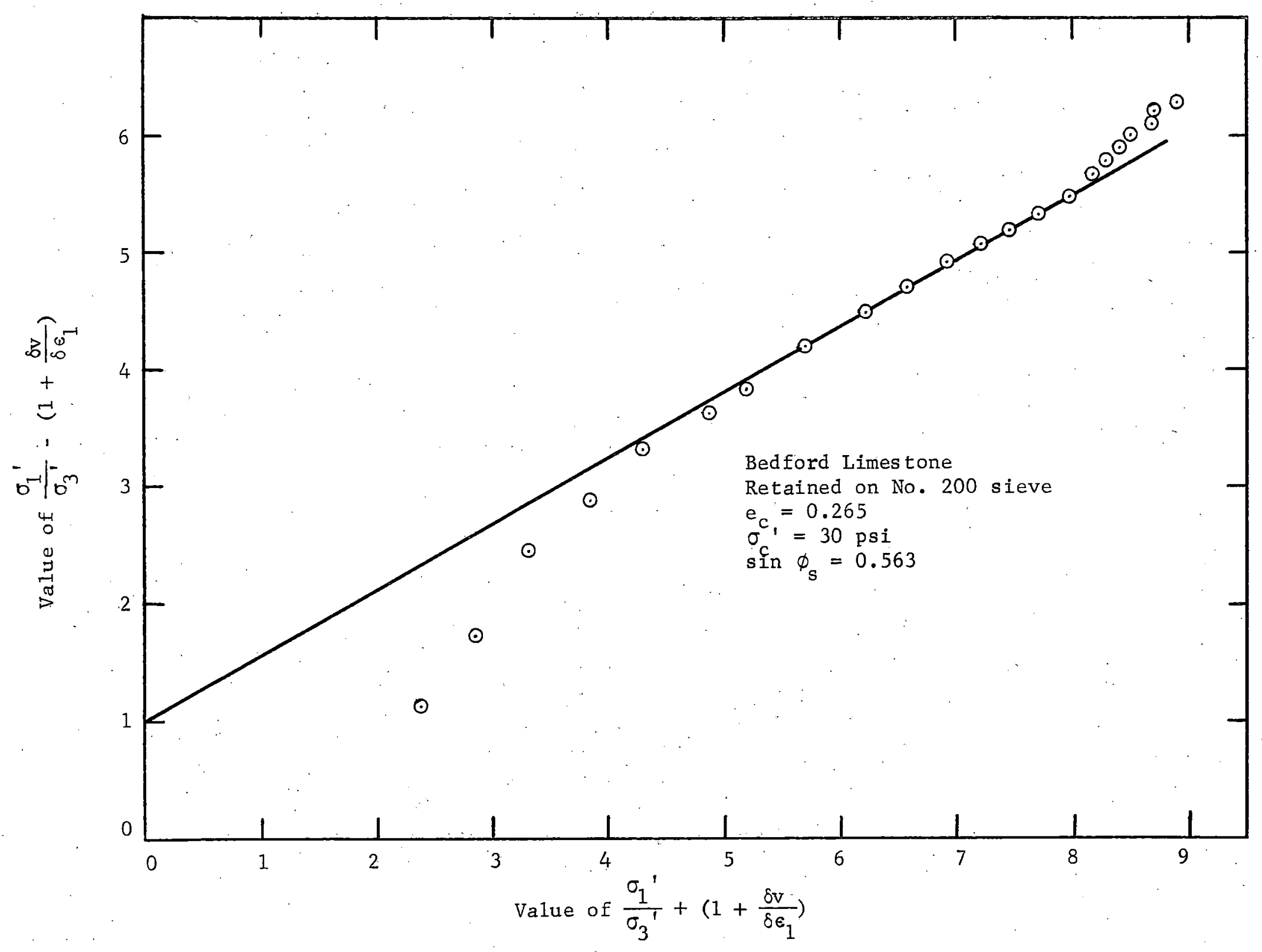

Fig. 29. Application of Eq. (9n) to tests on crushed limestones. 


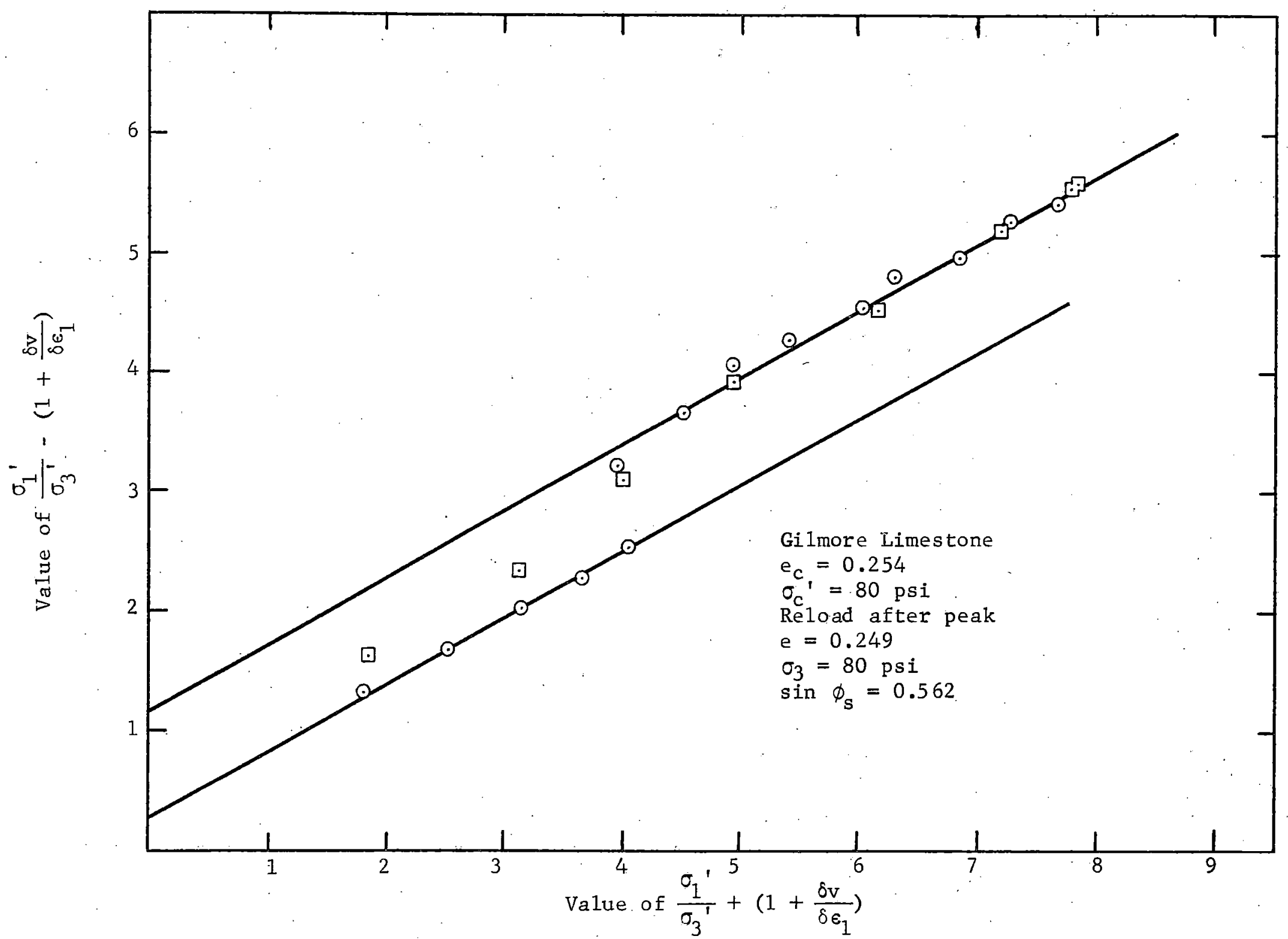

Fig. 30. Application of Eq. (9n) to tests on crushed limestones. 
Table 3. Summary of selected tests on crushed limestones.

\begin{tabular}{lccccc}
\hline Limestone & Figure & $\begin{array}{c}\text { Initial } \\
\text { void } \\
\text { ratio }\end{array}$ & $\begin{array}{c}\text { Confining } \\
\text { pressure } \\
\text { (psi) }\end{array}$ & $\begin{array}{c}\phi_{S} \\
\text { degrees }\end{array}$ & $\Omega$ \\
\hline Garner & 24 & 0.209 & 80 & $34.1^{\circ}$ & 1.45 \\
Bedford & 25 & 0.302 & 80 & 34.4 & 1.13 \\
Bedford & 26 & 0.266 & 80 & 34.4 & 1.04 \\
Garner & 27 & 0.197 & 30 & 34.1 & 2.18 \\
Bedford & 28 & 0.300 & 30 & 34.4 & 1.35 \\
Bedford & 29 & 0.265 & 30 & 34.2 & 1.00 \\
Gilmore & 30 & 0.254 & 80 & 34.2 & 1.15 \\
\hline
\end{tabular}

With reference to Table 3 , there is no obvious correlation between void ratio and the values of $\Omega$; or if there is a relation, it must be confounded by the influence of gradation and the co-functional variable, type of packing.

An influence of gradation on the values of $\Omega$ can be seen by comparing the values of $\Omega$ obtained from the crushed limestones with those obtained from the river sands (such a comparison may be made since $\Omega$, or rather $\Omega_{\mathrm{D}}$, is independent from the coefficient of solid friction between particles). The values of $\Omega$ are higher for the limestones than for the river sand (Table 1) at corresponding levels of confining pressures. The difference is larger at lower (30 psi) than at higher confining pressures, since the values of $\Omega$ for the limestones decreased as confining pressure increased. 
The larger values of $\Omega$ obtained from limestones compared to river sands may relate to the larger range in particle sizes, which could tend to increase the amount of particle rearrangement by rolling. That is, small particles in the crushed limestones may act as rollers between other individual particles or groups of particles. This effect would be reduced by increasing the confining pressure, due to an increase in the number of "fixed" contacts within the assembly. That is indicated by comparing the values of $\Omega$ given in Table 3 for Figs. 28 and 29. From Fig. 28, $\Omega=1.35$ for the whole Bedford sample tested at a confining pressure of 30 psi; from Fig. 29, $\Omega=1.00$ for a Bedford sample tested at the same confining pressure, but with the particles passing a US No. 200. sieve previously removed by dry sieving. This latter sample had a lower initial void ratio than did the former sample.

The stress history did not affect the values of $\Omega$ or the linear relation, as shown in Fig. 30. The effect of stress history was studied by unloading the sample after the maximum value of $\sigma_{1}{ }^{\prime} / \sigma_{3}{ }^{\prime}$ was reached and subsequently loading the sample to this maximum value.

\section{Deformation Stages}

Figures 31 to 35 show the ratio of the rate of internal work absorbed either in friction or in dilatancy to the rate of gross work input (represented by the product $\sigma_{1}^{\prime} \delta \epsilon_{1}$ ), as related to percent of axial strain. A feature common to all of the Figs. 31 to 35 is the increase of the frictional work ratio $\delta \mathrm{Wi}_{f} / \sigma_{1}{ }^{\prime} \delta \epsilon_{1}$, followed by sudden decreases during the initial stages of deformation, with this cycle being repeated to a lesser extent as the deformation increased. This 


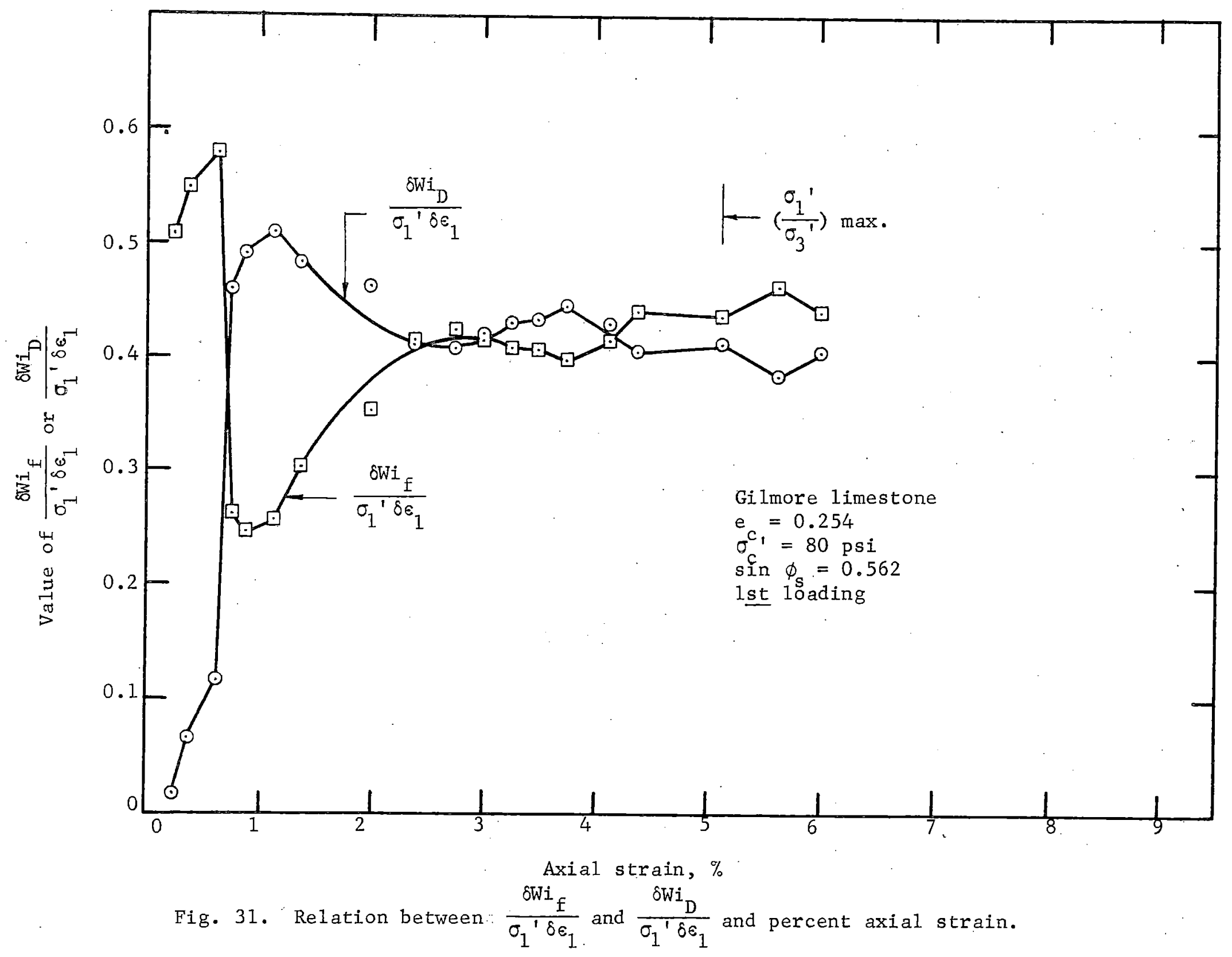




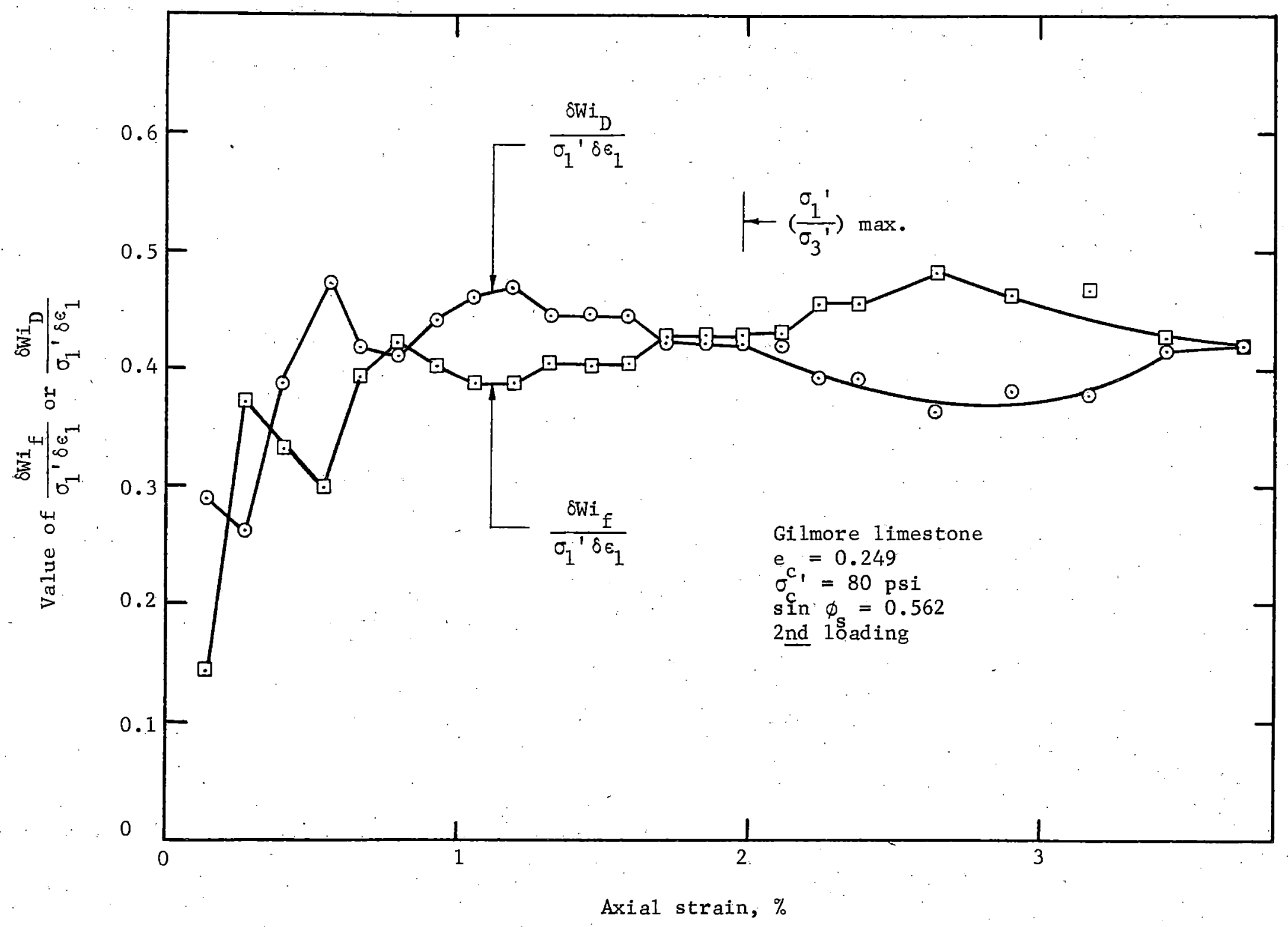

Fig. 32. Relation between $\frac{\delta W i_{f}}{\sigma_{1}^{\prime} \delta \epsilon_{1}}$ and $\frac{\delta W i_{D}}{\sigma_{1}^{\prime} \delta \epsilon_{1}}$ and percent axial strain. 


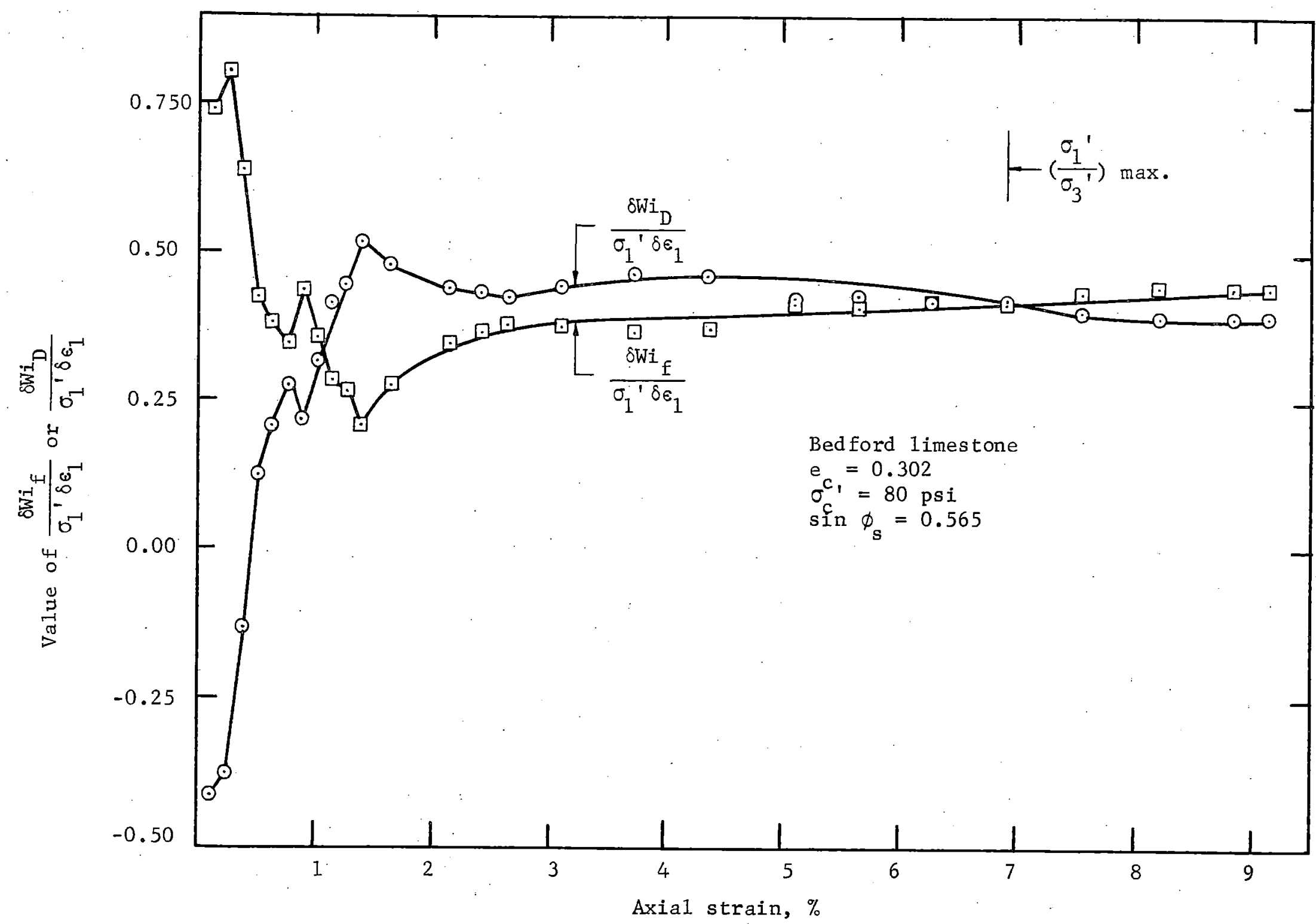

Fig. 33. Relation between $\frac{\delta \mathrm{Wi}_{f}}{\sigma_{1}^{\prime} \delta \varepsilon_{1}}$ and $\frac{\delta W i_{\mathrm{D}}}{\sigma_{1}^{\prime} \delta \epsilon_{1}}$ and percent axial strain. 


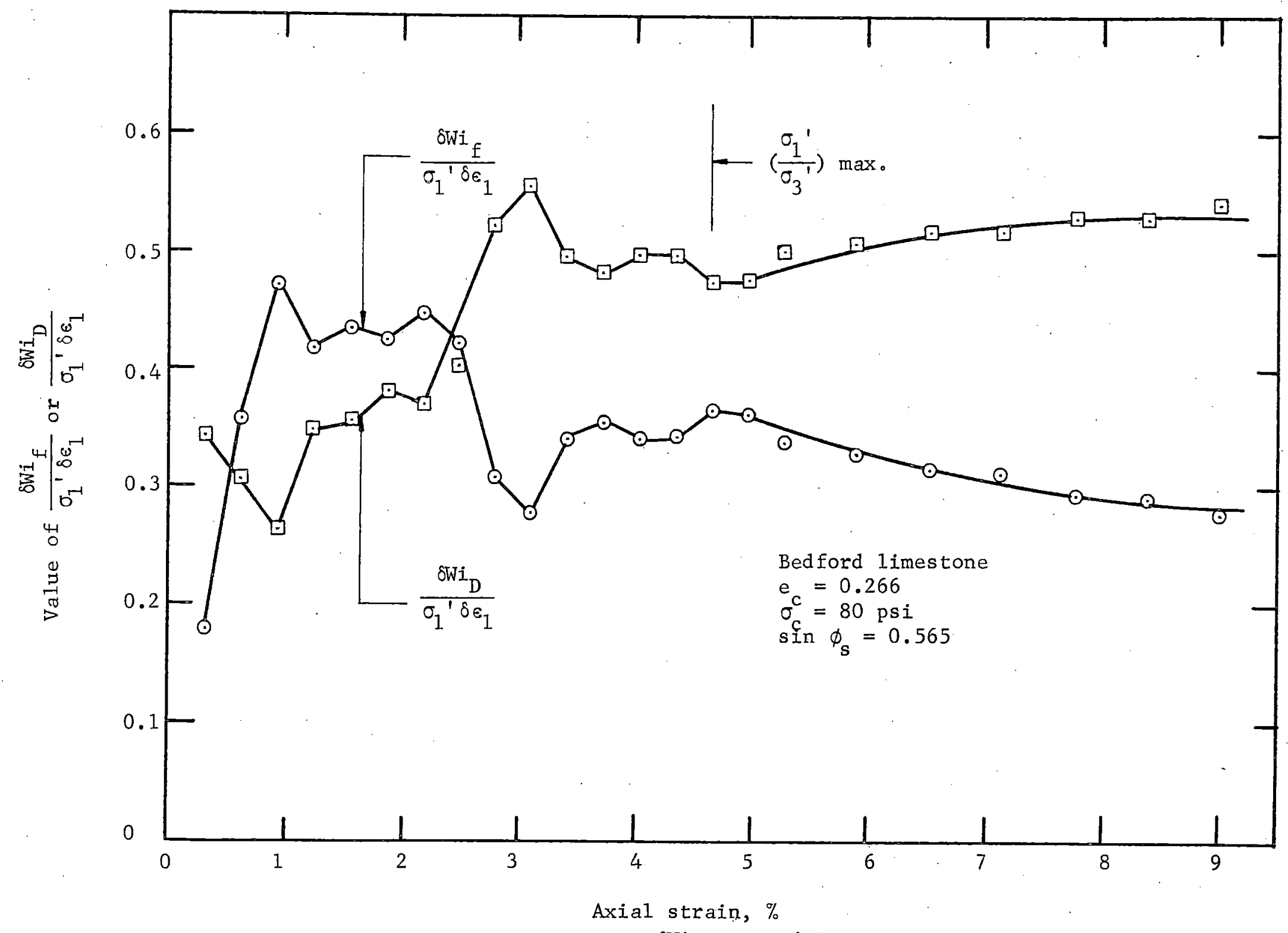

Fig. 34. Relation between $\frac{\delta W i_{f}}{\sigma_{1}^{\prime} \delta \varepsilon_{1}}$ and $\frac{\delta W i_{D}}{\sigma_{1}^{\prime} \delta \varepsilon_{1}}$ and percent axial strain. 


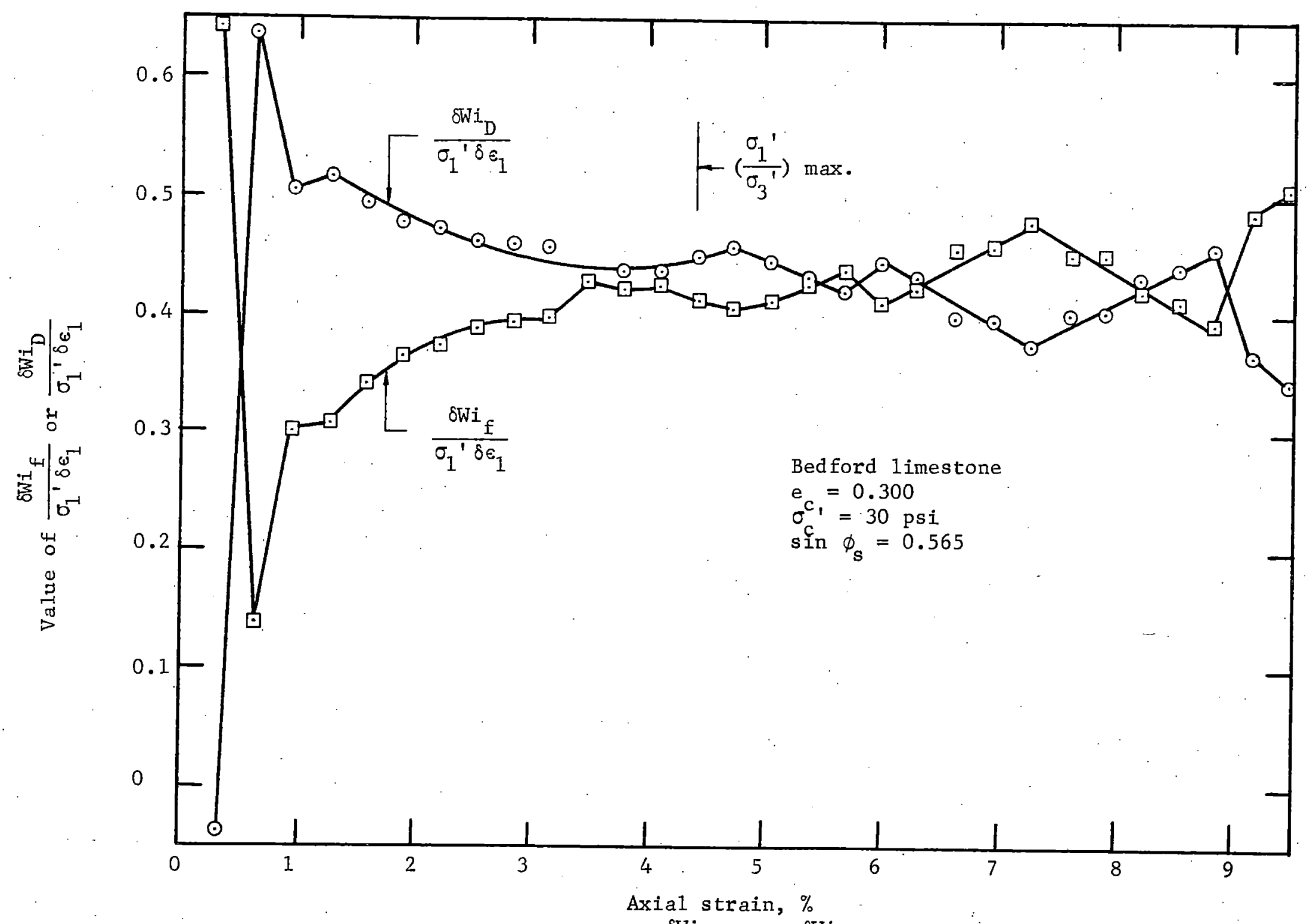

Fig. 35. Relation between $\frac{\delta W i_{f}}{\sigma_{1}^{\prime} \delta \epsilon_{1}}$ and $\frac{\delta W i_{D}}{\sigma_{1}^{\prime} \delta \epsilon_{1}}$ and percent axial strain. 
is accompanied by inverse trends in the dilational work ratio $\delta W_{D} / \sigma_{1}^{\prime} \delta \epsilon_{1} \cdot$

The initial increase in the values of $\delta \mathrm{Wi}_{f} / \sigma_{1}^{\prime} \delta \epsilon_{1}$ suggests an initial sliding between individual particles or groups of particles. Thus, deformation begins the moment that the ratio $\sigma_{1}{ }^{\prime} / \sigma_{3}^{\prime}$ exceeds unity. This deformation will consist of relative motions due to rolling and sliding among the particles, as reflected on increases of both $\delta W i_{f} / \sigma_{1}{ }^{\prime} \delta \epsilon_{1}$ and $\delta W i_{D} / \sigma_{1}^{\prime} \delta \epsilon_{1}$. Since instantaneous relative motions among all the particles within the assembly are not possible; there are four possible dissimilar actions between individual particles or groups of particles; namely, sliding, rolling, rotation, or breaking of the contact.

Certain number of contacts within the assembly are fixed, thereby forming instantaneous rigid groups of particles. Sliding therefore occurs only at the boundary surface of such groups, and all the contacts on the boundary surface must slide. Allowing a given group of particles to slide for a short distance before sliding ceases implies that the restraints produced by other groups must be such as to allow the motion to occur, i.e. the restraining contacts temporarily become rolling contacts, sliding contacts, or disappear (that is, break). Formation of new groups of contacts causes the slide of the original group to be completely arrested, and favors the formation of increased number of sliding contacts as reflected in increases of both $\delta W i_{f} / \sigma_{1}^{\prime} \delta \epsilon_{1}$ and $\delta W i_{D} / \sigma_{1}^{\prime} \delta \epsilon_{1}$. The continuous increase of $\delta W i_{f} / \sigma_{1}^{\prime} \delta \epsilon_{1}$ during the initial stages of deformation implies that slides are restricted to very small groups of particles. 
Eventually the number of sliding contacts is increased, 1arger groups of particles slide within the assembly, producing a sudden decrease in the ratio $\delta W i_{f} / \sigma_{1}^{\prime} \delta \epsilon_{1}$ with a corresponding sudden increase in the ratio $\delta \mathrm{Wi}_{D} / \sigma_{1}{ }^{\prime} \delta \varepsilon_{1}$. Still the slides between larger groups of particles must be arrested before they become catasgrophic (that is, formation of slip surface); most likely when a larger group of particles slides, the portion of the stresses carried out by this group is transferred to adjacent groups, inducing particle rearrangements and a sudden increase in the ratio $\delta W{ }_{\mathrm{D}} / \sigma_{1}{ }^{\prime} \delta \epsilon_{1}$ and the corresponding decrease in the ratio $\delta W_{\mathrm{f}} / \sigma_{1}{ }^{\prime} \delta \epsilon_{1}$. The arresting of the slide of a large group of particles is shown to have been accomplished by subsequent increase in the ratio $\delta W i_{f} / \sigma_{1}^{\prime} \delta \varepsilon_{1}$. This partial collapse within the assembly during the initial stages of deformation is the reason for the deviation from a linear relation between the terms given in Eq. (9n).

The postulated mechanism of deformation is not altered by reloading after the maximum value of $\sigma_{1}{ }^{\prime} / \sigma_{3}{ }^{\prime}$ is reached, as shown in Fig. 32. Increases in density and confining pressure did not alter the above postulated mechanism, as shown in Figs. 33 to 35 .

The behavior during the initial deformation stages, for which a low value of the ratio $\sigma_{1}{ }^{\prime} / \sigma_{3}{ }^{\prime}$ was applied, is probably much the same as occurs during compaction of a granular assembly. Further densification can only be achieved by sliding between group contacts and subsequent particle rearrangement to allow the formation of new groups and different sets of sliding contacts. The amount of energy required for further densification increases as the relative proportion of 
possible sliding contacts increases. Thus, vibrational methods of compaction are most successful in densification of granular materials because the process is to break the contacts and reduce the amount of sliding involved while promoting particle rearrangement by rolling and rotation.

\section{Maximum Stress Ratio and Initial Void Ratio}

Figure 36 shows the relation between the maximum value of $\sigma_{1}{ }^{\prime} / \sigma_{3}{ }^{\prime}$ and the initial void ratio for the three stones at different confining pressures. However, the slope of this linear relation decreases as the confining pressures increases, and becomes independent of the initial void ratio at a confining pressure of $80 \mathrm{psi}$ in the case of the Bedford samples. This suggests that the influence of the initial void ratio is confounded with the effect of the gradation and type of packing, since a different relationship was obtained for the Garner and Gilmore stones (Fig. 36). 


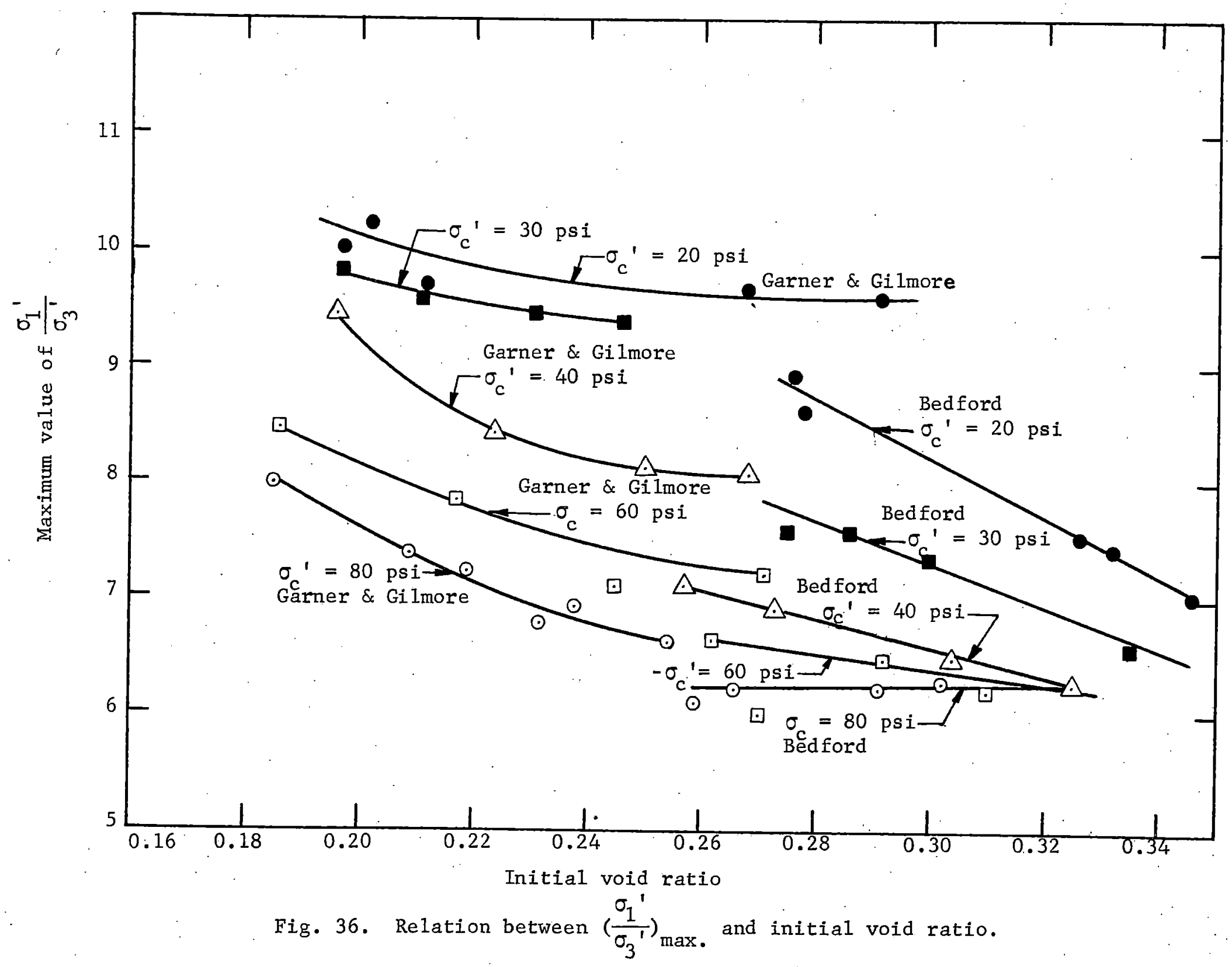




\section{CONCLUSIONS}

1. The theoretical equations developed in this report allowed the determination of the angle of solid friction between particles which was found to depend solely on the nature of the particle surface.

2. The separation of the frictional and dilational components of the shear strength of granular materials qualitatively corroborated the postulated mechanisms of deformation.

3. The influence of variables such as void ratio, gradation, packing, level of confining pressure, stress history, and type of shear test, on the shear strength of granular material was reflected in the values of the parameter $\Omega_{0}$ Stress Ratio

4. The determination of the coefficient of solid friction allows the establishment of a lower bound solution of the shear strength of granular materials. An upper bound solution cannot be established due to the dependence of the parameter $\Omega$ on test and boundary conditions. 


\section{ACKNOWLEDGEMENTS}

This research was part of a study of the factors influencing stability of granular base course mixes conducted at the Engineering Research Institute, Iowa State University, under sponsorship of the Iowa Highway Research Board, Iowa State Highway Commission, and Bureau of Public Roads, U. S. Department of Commerce.

The authors express their acknowledgement to Dr. Turgut Demire1, Associate Professor of Civil Engineering, for his guidance and counseling on energy concepts and related subjects. Special thanks are due to the staff of the Soil Research Laboratory, Engineering Research Institute, for their unselfish assistance during this study and to J. M. Hoover, Associate Professor of Civil Engineering, as director of the project. 


\section{REFERENCES}

Amontons. 1699. De 1a resistance cause'e dans les machines. Academie Royale des Sciences, Paris, France, Histoire avec les Mémoires de Mathematique et de Physique 1699:206-227.

Barden, I. and A. J. Khayatt. 1966. Incremental strain rate ratios and strength of sand in the triaxial test. Géotechnique 16:338-357.

Bishop, A. W. 1954. Correspondence (on a paper by A. D. M. Penman). Geotechnique 4:43-45.

Bishop, A. W. 1966. The strength of soils as engineering materials. Géotechnique 16:89-130.

Bishop, A. W. and A. K. G. Eldin. 1953. The effect of stress history on the relation between $\phi$ and porosity in sand. International Conference on Soil Mechanics and Foundations Engineering, 3rd, Zurich, Switzerland, Proceedings 1:100-105.

Bishop, A. W. and G. E. Green. 1965. The influence of end restraint on the compression strength of a cohesionless soil. Géotechnique 15: 243-266.

Bowden, F. P. and D. Tabor. 1950. The friction and lubrication of solids. London, England, Oxford University Press.

Cornforth, D. H. 1964: Some experiments on the influence of strain conditions on the strength of sand. Géotechnique 14:143-167.

Coulomb, C. A. 1781. Theorie des machines simples, en ayant égard au frottement de leurs parties, et a la roideur des cordages. Academie Royale des Sciences, Paris, France, Mémoires de Mathématique et de Physique 10, NO. 2:161-332. 1785.

Gibson, R. E. and N. R. Morgenstern. 1963. Stress-dilatancy, earth pressures, and slopes (by) P. W. Rowe: (discussion). American Society of Civil Engineers Proceedings 89 No. SM6:127-129.

Handy, R. L. 1965. Quantitative X-ray diffraction measurements by fast scanning. American Society for Testing and Materials Special Technical Publication 395:30-44.

Hoover, J. M. 1965. Factors influencing stability of granular base course mixes: final report. Ames, Iowa, Engineering Experiment Station, Iowa State University of Science and Technology.

Horn, H. M. 1961. An investigation of the frictional characteristics of minerals. Microfilm copy, unpublished Ph.D. thesis, University of Illinois. Urbana, Illinois. Ann Arbor, Michigan, University Microfilms, Inc. 
Horne, M. R. 1965. The behavior of an assembly of rotund, rigid, cohesionless particles. 1. Royal Society of London, London, England, Proceedings Series A, 286:62-78.

Lambe, T. W. 1951. Soil testing for engineers. New York, N.Y., Wiley and Sons, Inc.

Lee, I. K. 1966. Stress-dilatancy performance of feldspar. American Society of Civil Engineers Proceedings 92, No. SM2:79-103.

MacCurdy, E. 1938. The notebooks of Leonardo da Vinci. Vol. 1. New York, N.Y., Reynal and Hitchcock.

Newland, P. L. and G. H. Allely. 1957. Volume changes in drained triaxial tests on granular materials. Géotechnique 7:17-34.

Penman, A. D. M. 1953. Shear characteristics of a saturated silt. Geotechnique 3:312-328.

Reynolds, 0. 1885. On the dilatancy of media composed of rigid particles in contact. London, England, Philosophical Magazine Series $5,20: 469-481$.

Roscoe, K. H. and A. N. Schofield. 1964. Stress-dilatancy, earth pressures, and slopes (by) P. W. Rowe: (discussion). American Society of Civil Engineers Proceedings 90, No. SM1:136-150.

Rowe, P. W. 1962. The stress-dilatancy relation for static equilibrium of an assembly of particles in contact. Royal Society of London, London, England, Proceedings Series A, 269:500-529.

Rowe, P. W. 1963. Stress-dilatancy, earth pressures, and slopes. American Society of Civil Engineers Proceedings 89, No. SM3:37-61.

Rowe, P. W. 1964. Stress-dilatancy, earth pressures, and slopes (by) P. W. Rowe: (discussion: closure). American Society of Civil Engineers Proceedings 90 , No. SM4:145-180.

Rowe, P. W., L. Barden and I. K. Lee. 1964. Energy components during the triaxial cell and direct shear tests. Géotechnique 14:247-261.

Scott, R. F. 1964. Stress-dilatancy, earth pressures, and slopes (by) P. W. Rowe: (discussion). American Society of Civil Engineers Proceedings 90, No. SM1:133-135.

Skempton, A. W. and A. W. Bishop. 1950. Measurement of shear strength of soils. Géotechnique 2:90-108.

Taylor, D. W. 1948. Fundamentals of. soil mechanics. New York, N.Y., Wiley and Sons, Inc. 
Terzaghi, K. 1923. Die Berechnung der Durchlässikeitsziffer des Tones aus dem Verlauf der hydrodynamischen Spannungserscheinungen. Akademie der Wissenschaften, Wien, Österreisch, Mathematisch-naturwissenschaftiiche K1asse, Sitsungsberichte Part 2a, 132:125-138。

Terzaghi, K. 1925. Erdbaumechanik auf bodenphysikalischer Grundlage. Wien, Österreisch, F. Deuticke.

Trollope, D. H. and A. K. Parkin. 1963. Stress-dilatancy, earth pressures, and slopes (by) P. W. Rowe: (discussion). American Society of Civil Engineers Proceedings 89, No. SM6:129-133. 